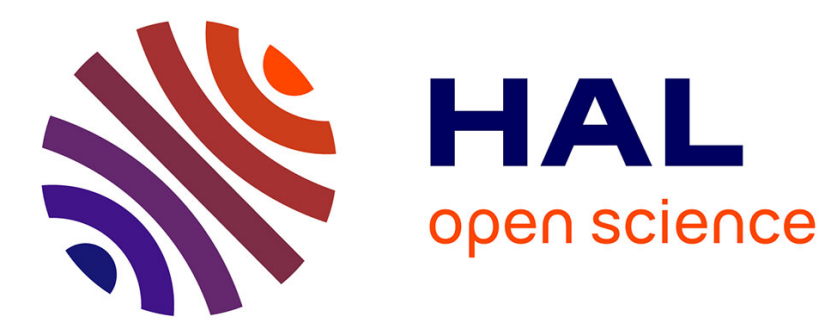

\title{
Torsion effects in elastic composites with high contrast
} Michel Bellieud

\section{To cite this version:}

Michel Bellieud. Torsion effects in elastic composites with high contrast. SIAM Journal on Mathematical Analysis, 2010, 41 (6), pp.2514-2553. hal-00777686

\section{HAL Id: hal-00777686 https://hal.science/hal-00777686}

Submitted on 17 Jan 2013

HAL is a multi-disciplinary open access archive for the deposit and dissemination of scientific research documents, whether they are published or not. The documents may come from teaching and research institutions in France or abroad, or from public or private research centers.
L'archive ouverte pluridisciplinaire HAL, est destinée au dépôt et à la diffusion de documents scientifiques de niveau recherche, publiés ou non, émanant des établissements d'enseignement et de recherche français ou étrangers, des laboratoires publics ou privés. 


\title{
TORSION EFFECTS IN ELASTIC COMPOSITES WITH HIGH CONTRAST
}

\author{
MICHEL BELLIEUD *
}

\begin{abstract}
We establish a homogenization result and a corrector result for a vibration problem of elasticity. We assume that the data depend in a periodic way on a small parameter $\varepsilon$. We assume also that the Lamé coefficients take possibly high values in a periodical set of disconnected inclusions and take values of the order $\varepsilon^{2}$ elsewhere. In the fibered case, torsional vibrations take place at an infinitesimal scale and give rise to non-local effects.
\end{abstract}

Key words. homogenization, elasticity, non-local effects

AMS subject classifications. 35B27, 35B40, 74B05, 74Q10

1. Introduction . In this paper, we analyze the behavior of solutions to initial boundary value problems describing vibrations of periodic elastic composites with rapidly varying elastic properties. More specifically, we analyze a two-phase medium whereby a set of "stiff" unbounded fibers or bounded inclusions is embedded in a "soft" matrix, i.e. what is often referred to as the "high contrast case". This task is set in the context of linearized elasticity.

Problems of a high-contrast type have been studied extensively over the last decades. Nowadays, there are two main trends in asymptotic methods: the asymptotic expansions and the two-scale convergence. The first approach [14], [25], [26], [29], [30] gives often stronger results including all asymptotic information about the solution and error estimates of higher order with respect to small parameters. It also contains the formulation of strong rigorous theories, but requires sufficiently regular data and boundaries. Let us mention in particular the detailed paper [28] of G. Sandrakov, yielding full proofs of the convergence and the error estimates for various high contrast asymptotic and geometric regimes in hyperbolic elastic problems. Let us mention also a most recent work [5] on the application of the asymptotic approach to some scalar spectral problems with high contrasts in both "stiffness" and "density", with rigorous convergence results and error bound obtained. The second approach [2], [4], [7], [9], [11], [13], [31] , employed in our paper, also yields the convergence to an asymptotic solution and a first order corrector result. It requires much less smoothness of the data but it does not allow to obtain any error estimates with respect to small parameters. Notice that the papers [13] and [14] apply the asymptotic expansions and the twoscale convergence respectively to the same problem: as a result, [13] ends with stronger results but for more regular boundaries.

We are aiming at complementing this extensive material. From the point of view of what is already available on the subject in the litterature, the most challenging case is that of a set of disconnected parallel fibers with elastic moduli of order 1 embedded in a "soft" matrix with moduli of order $\varepsilon^{2}$, where $\varepsilon$ is the period of the medium in the plane transverse to the fibers. We will focuse on the vibratory case. However, we emphasize that our analysis goes through in the same way in the case of equilibrium equations. The results obtained in this way are relevant to Example II and to Example III of the paper [9] by the author with G. Bouchitté, where fibered structures with elastic moduli respectively of order 1 and of order $\frac{1}{\varepsilon^{2}}$ embedded in a

* Département de Mathématiques, Université de Perpignan, 52 Av. Paul Alduy, 66860 Perpignan Cedex, France.(bellieud@univ-perp.fr). 
"soft" matrix were considered. We agree with the result obtained in Example III and we find that the result obtained in Example II is false. Indeed, the effective energy functional obtained in [9], Th. 2.4 turns out to be only a lower bound of the actual effective energy functional. We prove that the latter functional includes additional terms describing torsional stored energy (see Section 5). The study of the torsion effects is, essentially, the main new contribution our manuscript aims to target.

We turn now to a more detailed introduction of the paper. For a given bounded smooth open subset $\Omega$ of $\mathbb{R}^{3}$, we consider the vibration problem

$$
\left\{\begin{array}{l}
\rho_{\varepsilon} \frac{\partial^{2} \boldsymbol{u}_{\varepsilon}}{\partial t^{2}}-\operatorname{div}\left(\boldsymbol{\sigma}_{\varepsilon}\left(\boldsymbol{u}_{\varepsilon}\right)\right)=\rho_{\varepsilon} \boldsymbol{f} \text { in } \Omega \times(0, T),\left(\boldsymbol{f} \in L^{2}\left(0, T ; L^{2}\left(\Omega, \mathbb{R}^{3}\right)\right)\right), \\
\boldsymbol{\sigma}_{\varepsilon}\left(\boldsymbol{u}_{\varepsilon}\right)=\lambda_{\varepsilon} \operatorname{tr}\left(\boldsymbol{e}\left(\boldsymbol{u}_{\varepsilon}\right)\right) \boldsymbol{I}+2 \mu_{\varepsilon} \boldsymbol{e}\left(\boldsymbol{u}_{\varepsilon}\right), \quad \boldsymbol{e}\left(\boldsymbol{u}_{\varepsilon}\right)=\frac{1}{2}\left(\boldsymbol{\nabla} \boldsymbol{u}_{\varepsilon}+\nabla^{T} \boldsymbol{u}_{\varepsilon}\right), \\
\boldsymbol{u}_{\varepsilon} \in C\left(0, T ; H_{0}^{1}\left(\Omega, \mathbb{R}^{3}\right)\right) \cap C^{1}\left(0, T ; L^{2}\left(\Omega, \mathbb{R}^{3}\right)\right), \\
\boldsymbol{u}_{\varepsilon}(0)=\boldsymbol{a}_{0}, \quad \frac{\partial \boldsymbol{u}_{\varepsilon}}{\partial t}(0)=\boldsymbol{b}_{0},\left(\boldsymbol{a}_{0}, \boldsymbol{b}_{0}\right) \in H_{0}^{1}\left(\Omega, \mathbb{R}^{3}\right) \times L^{2}\left(\Omega, \mathbb{R}^{3}\right) .
\end{array}\right.
$$

We assume that the Lamé coefficients $\lambda_{\varepsilon}, \mu_{\varepsilon}$ take values of order 1 in an $\varepsilon$-periodic subset $B_{\varepsilon}$ of $\Omega$ consisting of parallel disjoint cylinders of Lebesgue measure of order 1 and take values of order $\varepsilon^{2}$ in the surrounding matrix. Heuristically, the norm of the gradient of the solution $\boldsymbol{u}_{\varepsilon}$ of (1.1) is expected to take high values, of the order $\frac{1}{\varepsilon}$, in the parts of the body where the coefficients are small. So, a gap between the mean displacement of the different constituent parts of the composite may take place, originating the non-local nature of the effective problem (see Remark 2.2 (i)). A commonly-used method consists in expressing the homogenized problem under the guise of a system of equations involving, besides the limit $\boldsymbol{u}_{0}$ of the sequence $\left(\boldsymbol{u}_{\varepsilon}\right)$, the limit $\boldsymbol{v}$ of an auxiliary sequence $\left(\boldsymbol{v}_{\varepsilon}\right)$ (see (2.16)) designed to characterize the average displacement in the inclusions. It turns out (see Theorem 2.1) that torsional vibrations take place at a microscopic scale in the fibers constituting the composite material. They are described in terms of the limit $\theta$ of the sequence $\left(\theta_{\varepsilon}\right)$ defined by (2.16), which characterizes the effective rescaled angle of torsion of the fibers (see Remark 2.2 (iv)). The functions $\boldsymbol{v}$ and $\theta$ are defined on $\Omega \times(0, T)$ and take values respectively in $\mathbb{R}^{3}$ and $\mathbb{R}$. The function $\boldsymbol{u}_{0}: \Omega \times(0, T) \times\left(-\frac{1}{2}, \frac{1}{2}\right)^{3} \rightarrow \mathbb{R}^{3}$ is the two-scale limit of $\left(\boldsymbol{u}_{\varepsilon}\right)$ (see [2], [23]). The effective displacement in the cylinders is governed by the coupled system of equations in $\Omega \times(0, T)$

$$
\left\{\begin{array}{l}
J^{\rho} \frac{\partial^{2} \theta}{\partial t^{2}}-k J \frac{\partial^{2} \theta}{\partial x_{3}^{2}}=\bar{\rho}_{1}\left(\left(\boldsymbol{y}_{G}-\boldsymbol{y}_{B}\right) \wedge\left(\boldsymbol{f}-\frac{\partial^{2} \boldsymbol{v}}{\partial t^{2}}\right)\right) \cdot \boldsymbol{e}_{3}+\boldsymbol{m}\left(\boldsymbol{u}_{0}\right) \cdot \boldsymbol{e}_{3} \\
\bar{\rho}_{1} \frac{\partial^{2} \boldsymbol{v}}{\partial t^{2}}-k|B| \frac{3 l+2}{l+1} \frac{\partial^{2} v_{3}}{\partial x_{3}^{2}} \boldsymbol{e}_{3}=\bar{\rho}_{1} \boldsymbol{f}+\boldsymbol{g}\left(\boldsymbol{u}_{0}\right)-\bar{\rho}_{1} \frac{\partial^{2} \theta}{\partial t^{2}} \boldsymbol{e}_{3} \wedge\left(\boldsymbol{y}_{G}-\boldsymbol{y}_{B}\right)
\end{array}\right.
$$

associated with the boundary and initial conditions given in (2.19), the constants $k$, $J^{\rho}, J, \boldsymbol{y}_{G}, \boldsymbol{y}_{B}, \bar{\rho}_{1}$ being defined by (2.2), (2.9), (2.12). The first equation of (1.2), regarding $\theta$, displays the torsional vibrations. The third component of the second equation shows extensional vibrations with regard to the longitudinal displacement $v_{3}$ (see [20], p. 428-429). The coupling with the matrix is marked by the fields $\boldsymbol{g}\left(\boldsymbol{u}_{0}\right)$ and $\boldsymbol{m}\left(\boldsymbol{u}_{0}\right)$. They represent respectively the sum of the surface forces applied on each fiber by the surrounding medium and their total moment with respect to the center 
of gravity of the geometric fiber. They are defined by (2.3), (2.4) in terms of the restriction to $\Omega \times(0, T) \times(Y \backslash B)$ of $\boldsymbol{u}_{0}$, which characterizes the effective displacement in the matrix. The letters $Y$ and $B$ symbolize respectively the unit cell and the rescaled fiber. The effective displacement in the matrix is governed by the equation

$$
\rho \frac{\partial^{2} \boldsymbol{u}_{0}}{\partial t^{2}}-\operatorname{div}_{y}\left(\boldsymbol{\sigma}_{0 y}\left(\boldsymbol{u}_{0}\right)\right)=\rho \boldsymbol{f} \quad \text { in } \Omega \times(0, T) \times(Y \backslash B),
$$

coupled with the variables $\boldsymbol{v}, \theta$ by the relation $\boldsymbol{u}_{0}=\boldsymbol{v}+\theta \boldsymbol{e}_{3} \wedge\left(\boldsymbol{y}-\boldsymbol{y}_{B}\right)$ in $B$, where $\rho$ stands for the strong two-scale limit of the mass density $\left(\rho_{\varepsilon}\right)$ and $\boldsymbol{\sigma}_{0 y}$ is defined by (2.3). The weak limit in $L^{2}$ of $\left(\boldsymbol{u}_{\varepsilon}\right)$ satisfies the non-explicit equation $\boldsymbol{u}(x, t)=$ $\int_{Y} \boldsymbol{u}_{0}(x, t, y) d y$. We obtain corrector results (see (2.25) and Remark 2.2 (iv)).

When the order of magnitude of the elasticity coefficients in the fibers is larger (namely when $k:=\lim _{\varepsilon \rightarrow 0} \mu_{1 \varepsilon}=+\infty$ ), the functions $\theta$ and $v_{3}$ are equal to zero and the effective displacement in the fibers is governed by the system of equations of $v_{1}, v_{2}$ given, in terms of the order of magnitude of the parameter $\kappa:=\lim _{\varepsilon \rightarrow 0} \varepsilon^{2} \mu_{1 \varepsilon}$, by (2.20), (2.21) or (2.22). In the most interesting case $0<\kappa<+\infty$, already investigated in the context of elliptic equations for fibers with a circular cross-section (see [9], Th. 2.5 ), this system involves the $4^{\text {th }}$ derivative of $v_{1}, v_{2}$ with respect to $x_{3}$, revealing bending effects (see [20], p. 430 ) similar to those studied in [10], [27]. Otherwise, the fibers display the behavior of a collection of unstretchable strings that do not twist if $\kappa=0$ and $k=+\infty$ and that of fixed bodies if $\kappa=\infty$.

If $B_{\varepsilon}$ consists of totally disconnected particles, the particles behave asymptotically like rigid bodies regardless of the order of magnitude $(\geq 1)$ of their stiffness. Their effective displacement is governed by the system of equations (3.6), where the field $\boldsymbol{r}$, obtained as the limit of the sequence $\left(\boldsymbol{r}_{\varepsilon}\right)$ defined by (3.3), describes their effective rotation vector (in the fibered case, $\boldsymbol{r}=\theta \boldsymbol{e}_{3}$ ). The displacement in the matrix is governed by the equation (1.3) coupled with $\boldsymbol{v}, \boldsymbol{r}$ by the equation $\boldsymbol{u}_{0}=\boldsymbol{v}+\boldsymbol{r} \wedge\left(\boldsymbol{y}-\boldsymbol{y}_{B}\right)$ in $\Omega \times(0, T) \times B$. Grain-like inclusions have been also considered by G. P. Panasenko [26] and G. V. Sandrakov [28] by using the asymptotic approach.

We can extend these results to the case of a multiphase medium comprising a finite collection $B_{\varepsilon}^{1}, . ., B_{\varepsilon}^{m}$ of non-intersecting $\varepsilon$-periodic families of grain-like inclusions or of fibers of various shapes and stiffness embedded in a "soft" matrix, each family of fibers being for simplicity parallel to one of the coordinate axes. The effective displacement in $B_{\varepsilon}^{i}$ is described in terms of a couple $\left(\boldsymbol{v}^{i}, \boldsymbol{r}^{i}\right)$ and governed by a system $\mathcal{P}^{\text {hom }}$ i similar, up to a rotation of the coordinate axes, to one of the systems (1.2), (2.20), (2.21), (2.22), (3.6) depending on the shape and on the order of magnitude of the elastic moduli in the specified inclusions (see Section 4 ). The displacement in the matrix is governed by the equation (1.3), where $B=B^{1} \cup \ldots \cup B^{m}$. The coupling of $\mathcal{P}^{\text {hom }} i$ with the matrix is marked by the equation $\boldsymbol{u}_{0}=\boldsymbol{v}^{i}+\boldsymbol{r}^{i} \wedge\left(\boldsymbol{y}-\boldsymbol{y}_{B^{i}}\right)$ in $B^{i}$ and by the presence of fields $\boldsymbol{g}^{i}\left(\boldsymbol{u}_{0}\right)$ and $\boldsymbol{m}^{i}\left(\boldsymbol{u}_{0}\right)$ in $\mathcal{P}^{\text {hom }} i$ (see Section 4). Multiphase homogenized models have been also considered in [25], [26], [28], [29], [30].

The two-phase models of composites obtained theoretically by our process of homogenization turn out to be unsufficiently reinforced, in general, to resist to some specific body forces. More precisely, in the elliptic case, the boundedness in $L^{2}\left(\Omega ; \mathbb{R}^{3}\right)$ of the solutions may fail to hold depending on $\boldsymbol{f}$ and, in the corresponding hyperbolic case, the effective equations may describe a motion of collapse. From a physical point of view, finding conditions ensuring the obtention of an effective elastic composite sufficiently reinforced to resist to body forces is an important task. We show (see Proposition 5.2) that the last mentioned boundedness is guaranteed for any choice 
of the field of body forces $f \in L^{2}\left(\Omega ; \mathbb{R}^{3}\right)$, if and only if a multiphase composite is considered whereby the set of inclusions comprises either one family of parallel fibers with elastic moduli of order $\frac{1}{\varepsilon^{2}}$, or three families of parallel fibers with elastic moduli of order 1 distributed in three independent directions. Hence, although two-phase media offer the convenient setting for the mathematical study of torsion effects, only multiphase media are likely to provide a physically satisfactory model of an elastic composite exhibiting torsion effects.

The paper is organised as follows: the notations and the results relating to the fibered case are displayed in Section 2, those concerning grain-like inclusions are stated in Section 3. The case of multiphase media and of equilibrium equations are discussed respectively in Section 4 and in Section 5. Section 6 is devoted mainly to a priori estimates in the fibered case. The proofs of the main Theorem 2.1 (fibered case) and Theorem 3.1 (case of grain-like inclusions) and a sketch of the proof of Proposition 5.2 are presented respectively in Section 7, Section 8 and Section 9.

2. Fibered case. In the sequel, $\left\{\boldsymbol{e}_{1}, \boldsymbol{e}_{2}, \boldsymbol{e}_{3}\right\}$ stands for the canonical basis of $\mathbb{R}^{3}$. Vectors and vector-valued functions are represented by symbols beginning by a boldface lower case letter (examples: $\boldsymbol{u}, \boldsymbol{f}, \boldsymbol{g}, \operatorname{div}(\boldsymbol{\sigma}), \ldots)$. For any vector $\boldsymbol{u} \in \mathbb{R}^{3}$, we denote by $u_{i}$ or $(\boldsymbol{u})_{i}$ its components (that is $\boldsymbol{u}=\sum_{i=1}^{3} u_{i} \boldsymbol{e}_{i}=\sum_{i=1}^{3}(\boldsymbol{u})_{i} \boldsymbol{e}_{i}$ ). We do not use the repeated index convention for summation. We denote by $\left(\varepsilon_{i j k}\right)$ the orientation tensor and by $\boldsymbol{u} \wedge \boldsymbol{v}=\sum_{i, j, k=1}^{3} \varepsilon_{i j k} u_{j} v_{k} \boldsymbol{e}_{i}$ the exterior product in $\mathbb{R}^{3}$. Matrices and matrix-valued functions are represented by symbols beginning by a boldface upper case letter with the following exceptions: $\boldsymbol{\nabla} \boldsymbol{u}$ (displacement gradient), $\boldsymbol{e}(\boldsymbol{u})$ (linearized strain tensor), $\boldsymbol{\sigma}(\boldsymbol{u})$ (linearized stress tensor). We denote by $\boldsymbol{A}: \boldsymbol{B}=$ $\sum_{i, j=1}^{3} A_{i j} B_{i j}$ the inner product of two matrices. We denote by $C$ different constants whose precise values may vary. Fixing a non-empty connected open set $D \subset \mathbb{R}^{2}$ with a Lipschitz boundary, we set

$$
\begin{aligned}
\rho \bar{D} \subset\left(-\frac{1}{2}, \frac{1}{2}\right)^{2}, B & :=D \times\left(-\frac{1}{2}, \frac{1}{2}\right), Y:=\left(-\frac{1}{2}, \frac{1}{2}\right)^{3}, \boldsymbol{y}:=\sum_{i=1}^{3} y_{i} \boldsymbol{e}_{i}, \\
|B| & :=\int_{B} d y, \boldsymbol{y}_{B}:=\frac{1}{|B|} \int_{B} \boldsymbol{y} d y, J:=\int_{B}\left|\boldsymbol{e}_{3} \wedge\left(\boldsymbol{y}-\boldsymbol{y}_{B}\right)\right|^{2} d y \\
J_{\alpha \beta}: & =\int_{B}\left(\boldsymbol{y}-\boldsymbol{y}_{B}\right)_{\alpha}\left(\boldsymbol{y}-\boldsymbol{y}_{B}\right)_{\beta} d y .
\end{aligned}
$$

Denoting by $\mathbb{S}^{3}$ the set of all real symmetric matrices of order 3 , we introduce the operators $\boldsymbol{e}_{y}, \boldsymbol{\sigma}_{0 y}: H^{1}\left(Y ; \mathbb{R}^{3}\right) \rightarrow L^{2}\left(Y ; \mathbb{S}^{3}\right), \boldsymbol{g}: \mathcal{H} \rightarrow \mathbb{R}^{3}, \boldsymbol{m}: \mathcal{H} \rightarrow \mathbb{R}^{3}$ defined by

$$
\begin{aligned}
& \left(\boldsymbol{e}_{y}(\boldsymbol{w})\right)_{i j}=\frac{1}{2}\left(\frac{\partial w_{i}}{\partial y_{j}}+\frac{\partial w_{j}}{\partial y_{i}}\right), \boldsymbol{\sigma}_{0 y}(\boldsymbol{w}):=\lambda_{0} \operatorname{tr}\left(\boldsymbol{e}_{y}(\boldsymbol{w})\right) \boldsymbol{I}+2 \mu_{0} \boldsymbol{e}_{y}(\boldsymbol{w}), \\
& \boldsymbol{g}(\boldsymbol{w}):=\int_{\partial B \cap Y} \boldsymbol{\sigma}_{0 y}(\boldsymbol{w}) \cdot \boldsymbol{n}_{B} d \mathcal{H}^{2}(y), \\
& \boldsymbol{m}(\boldsymbol{w}):=\int_{\partial B \cap Y}\left(\boldsymbol{y}-\boldsymbol{y}_{B}\right) \wedge\left(\boldsymbol{\sigma}_{0 y}(\boldsymbol{w}) \cdot \boldsymbol{n}_{B}\right) d \mathcal{H}^{2}(y),
\end{aligned}
$$

where $\boldsymbol{n}_{B}$ stands for the outward pointing normal to $\partial B, \lambda_{0}, \mu_{0}$ are positive reals, and 


$$
\mathcal{H}:=\left\{\boldsymbol{w} \in H^{1}\left(Y \backslash B ; \mathbb{R}^{3}\right), \operatorname{div}\left(\boldsymbol{\sigma}_{0 y}(\boldsymbol{w})\right) \in\left(H^{1}\left(Y \backslash B ; \mathbb{R}^{3}\right)\right)^{\prime}\right\},
$$

the symbol $E^{\prime}$ indicating the continuous dual of a Banach space $E$. We denote by $C_{\sharp}^{\infty}(Y)$ (resp. $C_{\sharp}(Y)$ ) the set of $Y$-periodic functions of $C^{\infty}\left(\mathbb{R}^{3}\right)$ (resp. $C\left(\mathbb{R}^{3}\right)$ ), by $C_{\sharp}^{\infty}(Y \backslash B)$ the set of the restrictions of the elements of $C_{\sharp}^{\infty}(Y)$ to $Y \backslash B$, by $H_{\sharp}^{1}(Y)$ (resp. $H_{\sharp}^{1}(Y \backslash B)$ ) the completion of $C_{\sharp}^{\infty}(Y)$ (resp. $C_{\sharp}^{\infty}(Y \backslash B)$ ) with respect to the norm $w \rightarrow\left(\int_{Y}\left(|w|^{2}+|\nabla w|^{2}\right) d y\right)^{\frac{1}{2}}\left(\right.$ resp. $\left.w \rightarrow\left(\int_{Y \backslash B}\left(|w|^{2}+|\nabla w|^{2}\right) d y\right)^{\frac{1}{2}}\right)$. Our proofs are based on the two-scale convergence method of G. Allaire [2] and G. Nguetseng [23] . A sequence $\left(f_{\varepsilon}\right)$ in $L^{2}\left(0, T ; L^{2}(\Omega)\right)$ is said to be two-scale convergent to $f_{0} \in L^{2}\left(0, T ; L^{2}(\Omega \times Y)\right.$ ) with respect to $x$ (notation: $f_{\varepsilon} \longrightarrow f_{0}$ ) if for each $\varphi_{0} \in \mathcal{D}\left(\Omega \times(0, T), C_{\sharp}^{\infty}(Y)\right)$, there holds

$$
\lim _{\varepsilon \rightarrow 0} \int_{\Omega \times(0, T)} f_{\varepsilon}(x, t) \varphi_{0}\left(x, t, \frac{x}{\varepsilon}\right) d x d t=\int_{\Omega \times(0, T) \times Y} f_{0} \varphi_{0} d x d t d y .
$$

A sequence $\left(\varphi_{\varepsilon}\right) \subset L^{2}\left(0, T ; L^{2}(\Omega)\right)$ is said to be two-scale strongly convergent to $\varphi_{0} \in L^{2}\left(0, T ; L^{2}(\Omega \times Y)\right)$ (notation $\left.\varphi_{\varepsilon} \longrightarrow \varphi_{0}\right)$ if

$$
\varphi_{\varepsilon} \longrightarrow \varphi_{0} \quad \text { and } \quad \lim _{\varepsilon \rightarrow 0}\left\|\varphi_{\varepsilon}\right\|_{L^{2}\left(0, T ; L^{2}(\Omega)\right)}=\left\|\varphi_{0}\right\|_{L^{2}\left(0, T ; L^{2}(\Omega \times Y)\right)} .
$$

The symbols $\longrightarrow$ and $\longrightarrow$ will be used also to denote the two-scale convergence and the strong two-scale convergence of sequences $\left(f_{\varepsilon}\right)$ in $L^{2}(\Omega)$ independent of $t$ or functions of $x$ only by formally regarding those as constant in $t$.

We consider the vibration problem (1.1), where $\Omega:=\omega \times 0, L, \omega$ is a bounded regular domain of $\mathbb{R}^{2}$ and $B_{\varepsilon}$ is the $\varepsilon$-periodic set of parallel cylinders defined by (see fig. 1)

$$
B_{\varepsilon}:=\Omega \cap \varepsilon \bigcup_{i \in \mathbb{Z}^{3}}(\{i\}+B) .
$$

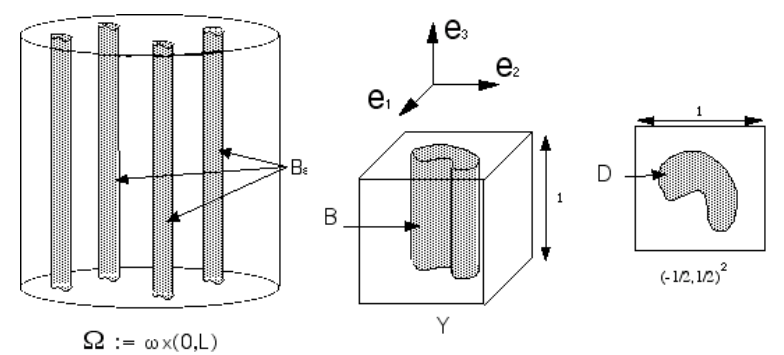

Fig. 1

We assume that the Lamé coefficients satisfy

$$
\begin{array}{ll}
\mu_{\varepsilon}(x)=\mu_{1 \varepsilon} 1_{B_{\varepsilon}}(x)+\varepsilon^{2} \mu_{0} 1_{\Omega \backslash B_{\varepsilon}}(x), & \lambda_{\varepsilon}(x)=\lambda_{1 \varepsilon} 1_{B_{\varepsilon}}(x)+\varepsilon^{2} \lambda_{0} 1_{\Omega \backslash B_{\varepsilon}}(x), \\
\mu_{1 \varepsilon} \geq c>0, \quad l_{\varepsilon}:=\frac{\lambda_{1 \varepsilon}}{\mu_{1 \varepsilon}}, & \lim _{\varepsilon \rightarrow 0} l_{\varepsilon}=l \in 0,+\infty .
\end{array}
$$


We set

$$
k:=\lim _{\varepsilon \rightarrow 0} \mu_{1 \varepsilon}, \quad \kappa:=\lim _{\varepsilon \rightarrow 0} \varepsilon^{2} \mu_{1 \varepsilon} .
$$

Under (2.8), the relative compactness of the sequence $\left(\boldsymbol{u}_{\varepsilon}\right)$ of the solutions of (1.1) in the $\star$-weak topology of $L^{\infty}\left(0, T ; L^{2}\left(\Omega ; \mathbb{R}^{3}\right)\right)$ is ensured by

$$
\begin{aligned}
& \boldsymbol{a}_{0}=0 \text { if } \mu_{1 \varepsilon}>>1, \quad 0 \leq \rho_{\varepsilon} \leq C<+\infty \text { if }\left\{\boldsymbol{b}_{0} \neq 0\right\} \text { or }\{\boldsymbol{f} \neq 0\}, \\
& \inf _{B_{\varepsilon}} \rho_{\varepsilon}>c>0 \quad \text { or } \quad \inf _{\Omega \backslash B_{\varepsilon}} \rho_{\varepsilon}>c>0, \quad \text { if } \kappa=0 .
\end{aligned}
$$

We suppose that

$$
\rho_{\varepsilon} \longrightarrow \rho,
$$

for some $\rho \in L^{2}(\Omega \times Y)$. The effective mass, the positions of the principal axes, the positions of the geometric principal axes and the moments of inertia with respect to the last mentioned axes of the fibers are characterized respectively by the constants $\bar{\rho}_{1}, \boldsymbol{y}_{G}, \boldsymbol{y}_{B}, J^{\rho}$ defined by $(2.2)$ and

$$
\begin{aligned}
\bar{\rho}_{1} & :=\int_{B} \rho d y, \quad \bar{\rho}_{1} \boldsymbol{y}_{G}:=\int_{B} \rho \boldsymbol{y} d y, \quad\left(\boldsymbol{y}_{G}=\boldsymbol{y}_{B} \text { if } \bar{\rho}_{1}=0\right), \\
J^{\rho} & :=\int_{B} \rho\left|\boldsymbol{e}_{3} \wedge\left(\boldsymbol{y}-\boldsymbol{y}_{B}\right)\right|^{2} d y
\end{aligned}
$$

We assume that (see Remark 2.2 (iii))

$$
D=\left\{\left(y_{1}, y_{2}\right) \in \mathbb{R}^{2}, \sqrt{y_{1}^{2}+y_{2}^{2}}<R\right\} \quad \text { if } \quad \liminf _{\varepsilon \rightarrow 0} \varepsilon \mu_{1 \varepsilon}<+\infty
$$

for some $R \in] 0, \frac{1}{2}[$. For simplicity the main result is stated under the additional hypotheses (see Remark $2.2(\mathrm{v})$ )

$$
\begin{gathered}
\rho_{\varepsilon} \geq c>0, \\
\rho_{\varepsilon} \leq C<+\infty .
\end{gathered}
$$

Representing by $\operatorname{Int}(s)$ the integer part of a real $s$, we set

$$
\begin{aligned}
& \boldsymbol{v}_{\varepsilon}(x, t):=\frac{1}{|B|} \boldsymbol{u}_{\varepsilon}(x, t) 1_{B_{\varepsilon}}(x) \\
& \theta_{\varepsilon}(x, t):=\frac{1}{J} \boldsymbol{u}_{\varepsilon}(x, t) .\left(\boldsymbol{e}_{3} \wedge\left(\left[\frac{\boldsymbol{x}}{\varepsilon}\right]-\boldsymbol{y}_{B}\right)\right) 1_{B_{\varepsilon}}(x) \\
& {\left[\frac{\boldsymbol{x}}{\varepsilon}\right]:=\sum_{i=1}^{3}\left[\frac{x_{i}}{\varepsilon}\right] \boldsymbol{e}_{i}, \quad\left[\frac{x_{i}}{\varepsilon}\right]:=\frac{x_{i}}{\varepsilon}-\left(\operatorname{Int}\left(\frac{x_{i}}{\varepsilon}+\frac{1}{2}\right)\right) .}
\end{aligned}
$$


Under these assumptions, we show that $\left(\boldsymbol{u}_{\varepsilon}, \boldsymbol{v}_{\varepsilon}, \theta_{\varepsilon}\right)$ converges, in the sense defined below, to $\left(\boldsymbol{u}_{0}, \boldsymbol{v}, \theta\right)$ (a geometrical interpretation of $\theta$ is given in Remark 2.2 (iv)) of

$$
\left\{\begin{array}{l}
\left(\mathcal{P}_{\text {matrix }}^{\text {hom }}\right), \\
\left(\mathcal{P}_{\text {fibers }}^{\text {hom }}(k, \kappa)\right),
\end{array}\right.
$$

where, setting $\boldsymbol{r}:=\theta \boldsymbol{e}_{3}$ and denoting by $\boldsymbol{n}$ the outward pointing normal to $\partial Y$,

$$
\left(\mathcal{P}_{\text {matrix }}^{\text {hom }}\right): \begin{cases}\rho \frac{\partial^{2} \boldsymbol{u}_{0}}{\partial t^{2}}-\operatorname{div}_{y}\left(\boldsymbol{\sigma}_{0 y}\left(\boldsymbol{u}_{0}\right)\right)=\rho \boldsymbol{f} & \text { in } \Omega \times(0, T) \times(Y \backslash B), \\ \boldsymbol{u}_{0}=\boldsymbol{v}+\boldsymbol{r} \wedge\left(\boldsymbol{y}-\boldsymbol{y}_{B}\right) & \text { in } \Omega \times(0, T) \times B, \\ \boldsymbol{\sigma}_{0 y}\left(\boldsymbol{u}_{0}\right) \cdot \boldsymbol{n}(y)=-\boldsymbol{\sigma}_{0 y}\left(\boldsymbol{u}_{0}\right) \cdot \boldsymbol{n}(-y) & \text { on } \Omega \times(0, T) \times \partial Y, \\ \boldsymbol{u}_{0} \in C\left([0, T] ; L^{2}\left(\Omega, H_{\sharp}^{1}\left(Y ; \mathbb{R}^{3}\right)\right)\right) \cap C^{1}\left([0, T] ; L^{2}\left(\Omega \times Y ; \mathbb{R}^{3}\right)\right), \\ \boldsymbol{u}_{0}(0) 1_{Y \backslash B}=\boldsymbol{a}_{0} 1_{Y \backslash B}, \quad \frac{\partial \boldsymbol{u}_{0}}{\partial t}(0) 1_{Y \backslash B}=\boldsymbol{b}_{0} 1_{Y \backslash B},\end{cases}
$$

and $\left(\mathcal{P}_{\text {fibers }}^{\text {hom }}(k, \kappa)\right)$ is given, in terms of the order of magnitude of the coefficients $k, \kappa$, by

$$
\begin{aligned}
& \underset{(0<k<+\infty)}{\left(\mathcal{P}_{\text {fibers }}^{\text {hom }}(k, 0)\right):}\left\{\begin{array}{l}
J^{\rho} \frac{\partial^{2} \theta}{\partial t^{2}}-k J \frac{\partial^{2} \theta}{\partial x_{3}^{2}} \\
=\bar{\rho}_{1}\left(\left(\boldsymbol{y}_{G}-\boldsymbol{y}_{B}\right) \wedge\left(\boldsymbol{f}-\frac{\partial^{2} \boldsymbol{v}}{\partial t^{2}}\right)\right) \cdot \boldsymbol{e}_{3}+\boldsymbol{m}\left(\boldsymbol{u}_{0}\right) \cdot \boldsymbol{e}_{3} \\
\bar{\rho}_{1} \frac{\partial^{2} \boldsymbol{v}}{\partial t^{2}}-k|B| \frac{3 l+2}{l+1} \frac{\partial^{2} v_{3}}{\partial x_{3}^{2}} \boldsymbol{e}_{3} \quad \text { in } \Omega \times(0, T), \\
=\bar{\rho}_{1} \boldsymbol{f}+\boldsymbol{g}\left(\boldsymbol{u}_{0}\right)-\bar{\rho}_{1} \frac{\partial^{2} \theta}{\partial t^{2}} \boldsymbol{e}_{3} \wedge\left(\boldsymbol{y}_{G}-\boldsymbol{y}_{B}\right) \quad \text { in } \Omega \times(0, T), \\
v_{3}, \theta \in C\left([0, T] ; L^{2}\left(\omega ; H_{0}^{1}(0, L)\right)\right) \cap C^{1}\left([0, T] ; L^{2}(\Omega)\right), \\
\boldsymbol{v} \in C^{1}\left([0, T] ; L^{2}\left(\Omega ; \mathbb{R}^{3}\right)\right), \\
\theta(0)=0, \frac{\partial \theta}{\partial t}(0)=0, \boldsymbol{v}(0)=\boldsymbol{a}_{0}, \frac{\partial \boldsymbol{v}}{\partial t}(0)=\boldsymbol{b}_{0},
\end{array}\right. \\
& \left(\mathcal{P}_{\text {fibers }}^{\text {hom }}(+\infty, 0)\right):\left\{\begin{array}{l}
\bar{\rho}_{1} \frac{\partial^{2} v_{\alpha}}{\partial t^{2}}=\bar{\rho}_{1} f_{\alpha}+\left(\boldsymbol{g}\left(\boldsymbol{u}_{0}\right)\right)_{\alpha} \quad \alpha \in\{1,2\} \quad \text { in } \Omega \times(0, T), \\
\boldsymbol{v} \in C^{1}\left([0, T], L^{2}\left(\Omega ; \mathbb{R}^{3}\right)\right), \\
v_{\alpha}(0)=0, \frac{\partial v_{\alpha}}{\partial t}(0)=\left(\boldsymbol{b}_{0}\right)_{\alpha}, \alpha \in\{1,2\}, v_{3}=\theta=0,
\end{array}\right. \\
& \begin{array}{l}
\left(\mathcal{P}_{\text {fibers }}^{\text {hom }}(+\infty, \kappa)\right): \\
(0<\kappa<+\infty)
\end{array}\left\{\begin{array}{c}
\bar{\rho}_{1} \frac{\partial^{2} v_{\alpha}}{\partial t^{2}}+\sum_{\beta=1}^{2} \kappa \frac{3 l+2}{l+1} J_{\alpha \beta} \frac{\partial^{4} v_{\beta}}{\partial x_{3}^{4}} \\
=\bar{\rho}_{1} f_{\alpha}+g_{\alpha}\left(\boldsymbol{u}_{0}\right), \alpha \in\{1,2\} \text { in } \Omega \times(0, T), \\
\boldsymbol{v} \in C\left([0, T] ; L^{2}\left(\omega, H_{0}^{2}\left(0, L ; \mathbb{R}^{3}\right)\right)\right) \cap C^{1}\left([0, T] ; L^{2}\left(\Omega ; \mathbb{R}^{3}\right)\right), \\
v_{\alpha}(0)=0, \frac{\partial v_{\alpha}}{\partial t}(0)=\left(\boldsymbol{b}_{0}\right)_{\alpha}, \alpha \in\{1,2\}, v_{3}=\theta=0,
\end{array}\right.
\end{aligned}
$$




$$
\left(\mathcal{P}_{\text {fibers }}^{\text {hom }}(+\infty,+\infty)\right): \quad \boldsymbol{v}=0, \quad \theta=0 .
$$

We establish the corrector result (2.25) under the assumption (see Remark 2.2 (iv))

$$
\boldsymbol{a}_{0}=0, \quad \boldsymbol{u}_{0}\left(x, t, \frac{x}{\varepsilon}\right) \longrightarrow \boldsymbol{u}_{0}
$$

Theorem 2.1. Assume (2.1), (2.7), (2.8), (2.10), (2.11), (2.13), (2.14), (2.15), let $\left(\boldsymbol{u}_{\varepsilon}\right)$ be the sequence of the solutions of (1.1) and let $\left(\boldsymbol{v}_{\varepsilon}\right),\left(\theta_{\varepsilon}\right)$ be defined by (2.16). Then $\left(\boldsymbol{u}_{\varepsilon}\right)$ two-scale converges to $\boldsymbol{u}_{0}$ with respect to $x$ and $\left(\boldsymbol{u}_{\varepsilon}, \boldsymbol{v}_{\varepsilon}, \theta_{\varepsilon}\right)$ converges starweakly in $\left(L^{\infty}\left(0, T ; L^{2}\left(\Omega, \mathbb{R}^{3}\right)\right)\right)^{2} \times L^{\infty}\left(0, T ; L^{2}(\Omega)\right)$ to $(\boldsymbol{u}, \boldsymbol{v}, \theta)$, where

$$
\begin{aligned}
& \boldsymbol{u}:=\int_{Y} \boldsymbol{u}_{0}(., y) d y, \quad \boldsymbol{v}=\int_{B} \boldsymbol{u}_{0}(., y) d y, \\
& \theta=\frac{1}{J} \int_{B} \boldsymbol{u}_{0}(., y) .\left(\boldsymbol{e}_{3} \wedge\left(\boldsymbol{y}-\boldsymbol{y}_{B}\right)\right) d y .
\end{aligned}
$$

The triple $\left(\boldsymbol{u}_{0}, \boldsymbol{v}, \theta\right)$ is the unique solution of (2.17). Moreover, $\left(\boldsymbol{u}_{\varepsilon}(\tau)\right)$ two-scale converges to $\boldsymbol{u}_{0}(\tau)$ with respect to $x$, for each $\tau \in 0, T$. Assume in addition (2.23), then $\left(\boldsymbol{u}_{\varepsilon}\right)$ two-scale converges strongly to $\boldsymbol{u}_{0}$ and

$$
\lim _{\varepsilon \rightarrow 0}\left\|\boldsymbol{u}_{\varepsilon}-\boldsymbol{u}_{0}\left(x, t, \frac{x}{\varepsilon}\right)\right\|_{L^{2}\left(\Omega \times(0, T) ; \mathbb{R}^{3}\right)}=0 .
$$

Remark 2.2. (i) If $0<k<+\infty$, the variable $\theta$ satisfies the vibrating string equation $\frac{\partial^{2} \theta}{\partial t^{2}}-c^{2} \frac{\partial^{2} \theta}{\partial x_{3}^{2}}=h, \theta(0)=\frac{\partial \theta}{\partial t}(0)=0, \theta\left(x^{\prime}, 0, t\right)=\theta\left(x^{\prime}, L, t\right)=0$, where $c:=\sqrt{\frac{k J}{J}}$, $h:=\frac{1}{J^{\rho}}\left(\bar{\rho}_{1}\left(\left(\boldsymbol{y}_{G}-\boldsymbol{y}_{B}\right) \wedge \boldsymbol{f}\right) \cdot e_{3}+\boldsymbol{m}\left(\boldsymbol{u}_{0}\right) \cdot \boldsymbol{e}_{3}-\bar{\rho}_{1}\left(\left(\boldsymbol{y}_{G}-\boldsymbol{y}_{B}\right) \wedge \frac{\partial^{2} \boldsymbol{v}}{\partial t^{2}}\right) \cdot \boldsymbol{e}_{3}\right)$, hence is given by

$$
\begin{aligned}
& \theta(x, t)=\sum_{n=1}^{+\infty} \frac{L}{c n \pi}\left(\int_{0}^{t} \sin \left(\frac{c n \pi}{L}(t-\tau)\right) \gamma_{n}\left(x_{1}, x_{2}, \tau\right) d \tau\right) \sqrt{\frac{2}{L}} \sin \left(\frac{n \pi}{L} x_{3}\right), \\
& \gamma_{n}\left(x_{1}, x_{2}, t\right)=\int_{0}^{L} h\left(x_{1}, x_{2}, x_{3}, t\right) \sqrt{\frac{2}{L}} \sin \left(\frac{n \pi}{L} x_{3}\right) d x_{3} .
\end{aligned}
$$

The substitution of (2.26) in (2.18), (2.19) reveals the presence of memory terms in the limit problem. Memory effects induced by homogenization are studied also in [1], [3], [21], [32]. More generally, non-local effects are likely to come about in composites with high contrast [2], [4]-[7], [9]-[11], [13], [14], [17], [25], [28]-[31]. In the case of scalar linear elliptic equations, they can be interpreted in the context of Dirichlet forms [22]. This approach breaks down in the framework of linear elasticity, any non-negative lower-semicontinuous quadratic form on $L^{2}\left(\Omega ; \mathbb{R}^{3}\right)$ being theoretically the limit of a suitable sequence of linear elasticity functionals on $H^{1}\left(\Omega ; \mathbb{R}^{3}\right)$ [12]. Passing from stationary to evolution equations, memory effects can add further to the possible non-local effects attendant on the elliptic case, even though the homogenization of the corresponding equilibrium equations leads to a classical local problem [10], Remark 3.2; [7], Remark 2.2 (v). 
(ii) If the fibers have a vanishing measure (i.e. $r_{\varepsilon}<<\varepsilon$, where $r_{\varepsilon}$ stands for their diameter) and if the elastic moduli are of order 1 in the matrix, a similar effective behavior is obtained in the inclusions, conditioned by $\tilde{k}:=\lim _{\varepsilon \rightarrow 0} \frac{r_{\varepsilon}^{2}}{\varepsilon^{2}} \mu_{1 \varepsilon}$ and $\tilde{\kappa}:=$ $\lim _{\varepsilon \rightarrow 0} \frac{r_{\varepsilon}^{4}}{\varepsilon^{2}} \mu_{1 \varepsilon}$, provided $0<\lim _{\varepsilon \rightarrow 0} \frac{1}{\varepsilon^{2}\left|\log \left(r_{\varepsilon}\right)\right|}<+\infty$. However, no torsional vibrations take place when $0<\tilde{k}<+\infty$ [10], Theorem 3.1.

(iii) If $\lim _{\varepsilon \rightarrow 0} \varepsilon \mu_{1 \varepsilon}=0$, the attempt to extend our result to the case of fibers with non circular cross sections leads to a technical complication (see Lemma 6.5).

(iv) Assumption (2.23) is verified for instance when $\boldsymbol{u}_{0}$ is continuous in at least one of the variables $(x, t)$ or $y$ (see [2], Section 5 ), which takes place provided $\boldsymbol{b}_{0}, \boldsymbol{f}, \rho$ are sufficiently regular. Under (2.23), the corrector result (2.25), combined with the second line of (2.18), indicates that the field $\boldsymbol{v}(x, t)+\theta(x, t) \boldsymbol{e}_{3} \wedge\left(\left[\frac{\boldsymbol{x}}{\varepsilon}\right]-\boldsymbol{y}_{B}\right)$ approximates the displacement in the fibers. Hence the function $\frac{\theta}{\varepsilon}$ is a local approximation of the microscopic rotation angle of the fibers. We can deduce also from (2.25) (the details are omitted) that the sequences $\left(\overline{\boldsymbol{v}}_{\varepsilon}\right)$ and $\left(\bar{\theta}_{\varepsilon}\right)$ obtained by averaging $\boldsymbol{v}_{\varepsilon}$ and $\theta_{\varepsilon}$ on each periodicity cell, namely

$$
\begin{aligned}
& \overline{\boldsymbol{v}}_{\varepsilon}(x, t):=\sum_{i \in I_{\varepsilon}}\left(f_{Y_{\varepsilon}^{i}} \boldsymbol{v}_{\varepsilon}(s, t) d s\right) 1_{Y_{\varepsilon}^{i}}(x), \\
& \bar{\theta}_{\varepsilon}(x, t):=\sum_{i \in I_{\varepsilon}}\left(f_{Y_{\varepsilon}^{i}} \theta_{\varepsilon}(s, t) d s\right) 1_{Y_{\varepsilon}^{i}}(x), \\
& Y_{\varepsilon}^{i}:=\varepsilon(i+Y), \quad I_{\varepsilon}:=\left\{i \in Z^{3}, Y_{\varepsilon}^{i} \subset \Omega\right\},
\end{aligned}
$$

converge respectively strongly to $\boldsymbol{v}$ in $L^{2}\left(0, T ; L^{2}\left(\Omega ; \mathbb{R}^{3}\right)\right)$ and strongly to $\theta$ in $L^{2}(0, T$; $\left.L^{2}(\Omega)\right)$.

(v) If (2.15) fails to hold, the effective displacement is stationary in the parts of the body where $\rho_{\varepsilon} \gg 1$ (see (6.49)). If (2.14) is not satisfied, some modifications of the data related to time in (2.17) are possibly required.

3. Case of grain-like inclusions . In this section, we assume that $\Omega$ and $B$ are regular domains of $\mathbb{R}^{3}$, and that (see fig. 2)

$$
\bar{B} \subset Y:=\left(-\frac{1}{2}, \frac{1}{2}\right)^{3} .
$$

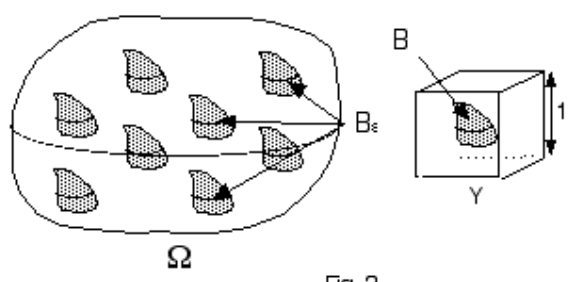

Fig. 2

The relative compactness of the sequence of the solutions of (1.1) in the $\star$-weak topology of $L^{\infty}\left(0, T ; L^{2}\left(\Omega ; \mathbb{R}^{3}\right)\right)$ is ensured by the assumptions $(2.8),(2.10)$ and 


$$
\inf _{B_{\varepsilon}} \rho_{\varepsilon}>c>0 \quad \text { or } \quad \inf _{\Omega \backslash B_{\varepsilon}} \rho_{\varepsilon}>c>0 .
$$

We introduce the inertia matrices $\boldsymbol{J}^{\rho}, \boldsymbol{J}$ and the sequence $\left(\boldsymbol{r}_{\varepsilon}\right)$ given by

$$
\begin{aligned}
J_{i j}^{\rho} & :=-\int_{B} \rho\left(\boldsymbol{y}-\boldsymbol{y}_{B}\right)_{i}\left(\boldsymbol{y}-\boldsymbol{y}_{B}\right)_{j} d y, \quad \text { if } \quad i \neq j, \\
J_{i j} & :=-\int_{B}\left(\boldsymbol{y}-\boldsymbol{y}_{B}\right)_{i}\left(\boldsymbol{y}-\boldsymbol{y}_{B}\right)_{j} d y, \quad \text { if } \quad i \neq j, \\
J_{i i}^{\rho} & :=\sum_{j \neq i} \int_{B} \rho\left|\left(\boldsymbol{y}-\boldsymbol{y}_{B}\right)_{j}\right|^{2} d y, \quad J_{i i}:=\sum_{j \neq i} \int_{B}\left|\left(\boldsymbol{y}-\boldsymbol{y}_{B}\right)_{j}\right|^{2} d y, \\
\boldsymbol{r}_{\varepsilon} & :=\boldsymbol{J}^{-1}\left(\left(\left[\frac{\boldsymbol{x}}{\varepsilon}\right]-\boldsymbol{y}_{B}\right) \wedge \boldsymbol{u}_{\varepsilon}\right) 1_{B_{\varepsilon}} .
\end{aligned}
$$

Theorem 3.1. Assume (2.8), (2.10), (2.11), (2.14), (2.15), (3.1), (3.2), let ( $\left.\boldsymbol{u}_{\varepsilon}\right)$ be the sequence of the solutions of (1.1) and let $\left(\boldsymbol{v}_{\varepsilon}\right),\left(\boldsymbol{r}_{\varepsilon}\right)$ be defined by (2.16), (3.3). Then the sequence $\left(\boldsymbol{u}_{\varepsilon}\right)$ two-scale converges to $\boldsymbol{u}_{0}$ with respect to $x$ and the sequence $\left(\boldsymbol{u}_{\varepsilon}, \boldsymbol{v}_{\varepsilon}, \boldsymbol{r}_{\varepsilon}\right)$ converges star-weakly in $\left(L^{\infty}\left(0, T ; L^{2}\left(\Omega, \mathbb{R}^{3}\right)\right)\right)^{3}$ to the triple $(\boldsymbol{u}, \boldsymbol{v}, \boldsymbol{r})$ given by (2.24) and

$$
\boldsymbol{r}=\boldsymbol{J}^{-1}\left(\int_{B}\left(\boldsymbol{y}-\boldsymbol{y}_{B}\right) \wedge \boldsymbol{u}_{0}(., y) d y\right) .
$$

The triple $\left(\boldsymbol{u}_{0}, \boldsymbol{v}, \boldsymbol{r}\right)$ is the unique solution of the system

$$
\left\{\begin{array}{l}
\left(\mathcal{P}_{\text {matrix }}^{\text {hom }}\right), \\
\left(\mathcal{P}_{\text {inclusions }}^{\text {hom }}\right),
\end{array}\right.
$$

where $\left(\mathcal{P}_{\text {matrix }}^{\text {hom }}\right)$ is given by (2.18) and

$$
\left(\mathcal{P}_{\text {inclusions }}^{\text {hom }}\right):\left\{\begin{array}{c}
\bar{\rho}_{1}\left(\frac{\partial^{2} \boldsymbol{v}}{\partial t^{2}}+\frac{\partial^{2} \boldsymbol{r}}{\partial t^{2}} \wedge\left(\boldsymbol{y}_{G}-\boldsymbol{y}_{B}\right)\right) \\
=\bar{\rho}_{1} \boldsymbol{f}+\boldsymbol{g}\left(\boldsymbol{u}_{0}\right) \quad \text { in } \Omega \times(0, T), \\
\boldsymbol{J}^{\rho} \cdot \frac{\partial^{2} \boldsymbol{r}}{\partial t^{2}}+\bar{\rho}_{1}\left(\boldsymbol{y}_{G}-\boldsymbol{y}_{B}\right) \wedge \frac{\partial^{2} \boldsymbol{v}}{\partial t^{2}} \\
=\bar{\rho}_{1}\left(\boldsymbol{y}_{G}-\boldsymbol{y}_{B}\right) \wedge \boldsymbol{f}+\boldsymbol{m}\left(\boldsymbol{u}_{0}\right) \quad \text { in } \Omega \times(0, T), \\
\boldsymbol{v}, \boldsymbol{r} \in C^{1}\left(0, T ; L^{2}\left(\Omega ; \mathbb{R}^{3}\right)\right), \\
\boldsymbol{v}(0)=\boldsymbol{a}_{0}, \quad \frac{\partial \boldsymbol{v}}{\partial t}(0)=\boldsymbol{b}_{0}, \quad \boldsymbol{r}(0)=\frac{\partial \boldsymbol{r}}{\partial t}(0)=0 .
\end{array}\right.
$$

Moreover, $\left(\boldsymbol{u}_{\varepsilon}(\tau)\right)$ two-scale converges to $\boldsymbol{u}_{0}(\tau)$ for each $\tau \in[0, T]$. Assume in addition (2.23), then $\left(\boldsymbol{u}_{\varepsilon}\right)$ two-scale converges strongly to $\boldsymbol{u}_{0}$ and the corrector result (2.25) holds.

Remark 3.2. (i) Grain-like inclusions are concerned as well with Remark 2.2 (i), (iv), (v). Regarding (ii), memory effects are obtained with particles of high mass density and diameter $r_{\varepsilon}<<\varepsilon$, provided $0<\lim _{\varepsilon \rightarrow 0} \frac{r_{\varepsilon}}{\varepsilon^{3}}<+\infty$ (see [7], [8]). 
(ii) In the fibered case, the sequence $\left(\boldsymbol{r}_{\varepsilon}\right)$ defined by (3.3) converges to $\theta \boldsymbol{e}_{3}$ (see Remark 8.1).

4. Multiphase media. We can extend our results easily to the case of a multiphase medium whereby $m \varepsilon$-periodic disconnected families $B_{\varepsilon}^{1}, \ldots, B_{\varepsilon}^{m}$ of fibers which are (for simplicity) assumed parallel to one of the coordinate axes or of grain-like inclusions are embedded in a soft matrix. The sets $B_{\varepsilon}^{1}, \ldots, B_{\varepsilon}^{m}$ are described in terms of $m$ subsets $B^{1}, . ., B^{m}$ of $Y$, connected in $\mathbb{R}^{3}$ and with disjoint closures, by setting $B_{\varepsilon}:=\bigcup_{i=1}^{m} B_{\varepsilon}^{i}, \quad B_{\varepsilon}^{i}:=\varepsilon\left(\bigcup_{j \in \mathbb{Z}^{3}} j+B^{i}\right) \cap \Omega, \quad B:=\bigcup_{i=1}^{m} B^{i}$ (see fig. 3 ). In the fibered case, $B^{i}$ is a cylinder whose axis is perpendicular to some face of the cube $Y$ (see fig. 3).

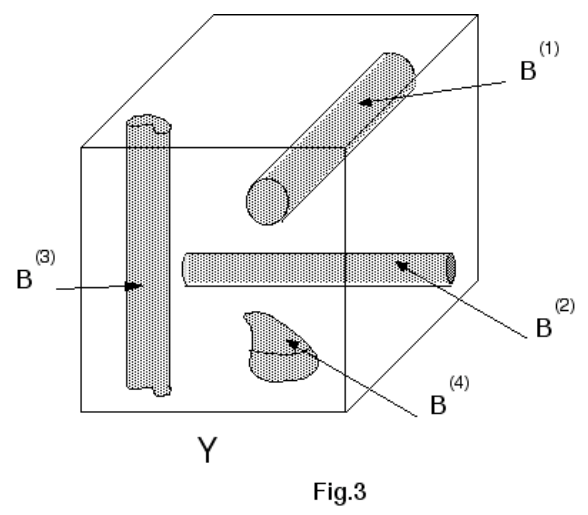

We suppose that the elastic moduli take the value $\mu_{1 \varepsilon}^{i}$ and $\lambda_{1 \varepsilon}^{i}$ on each set $B_{\varepsilon}^{i}(i \in$ $\{1, \ldots, m\})$ and take the value $\varepsilon^{2} \mu_{0}$ and $\varepsilon^{2} \lambda_{0}$ in the matrix $\Omega \backslash B_{\varepsilon}$. By repeating the argument of the proof of Theorem 3.1 and Theorem 3.2, we find that the sequence $\left(\boldsymbol{u}_{\varepsilon}\right)$ of the solutions of (1.1) two-scale converges to the unique solution $\boldsymbol{u}_{0}$ of the following equivalent variational problem

$$
\begin{aligned}
& \int_{0}^{T}\left(a\left(\boldsymbol{u}_{0}(t), \boldsymbol{w}_{0}\right) \eta(t)+\left(\boldsymbol{u}_{0}(t), \boldsymbol{w}_{0}\right)_{H} \eta^{\prime \prime}(t)\right) d t+\left(\boldsymbol{a}_{0}, \boldsymbol{w}_{0}\right)_{H} \eta^{\prime}(0) \\
& \quad-\left(\boldsymbol{b}_{0}, \boldsymbol{w}_{0}\right)_{H} \eta(0)=\int_{0}^{T}\left(\boldsymbol{f}, \boldsymbol{w}_{0}\right)_{H} \eta(t) d t, \quad \forall \boldsymbol{w}_{0} \in V, \quad \forall \eta \in \mathcal{D}(-\infty, T), \\
& \boldsymbol{v}, \boldsymbol{r} \in C^{1}\left(0, T ; L^{2}\left(\Omega ; \mathbb{R}^{3}\right)\right), \\
& \boldsymbol{u}_{0} \in L^{2}(0, T ; V), \boldsymbol{u}_{0}^{\prime} \in L^{2}(0, T ; H) .
\end{aligned}
$$

The Hilbert space $H$ is the set of all $\boldsymbol{w}_{0} \in L^{2}\left(\Omega ; L_{\sharp}^{2}\left(Y ; \mathbb{R}^{3}\right)\right)$ such that for each $i \in$ $\{1,, \ldots, m\}$, there exists a couple $\left(\boldsymbol{\psi}^{[i]}, \boldsymbol{r}^{[i]}\right) \in\left(L^{2}\left(\Omega ; \mathbb{R}^{3}\right)\right)^{2}$ such that $\boldsymbol{w}_{0}=\boldsymbol{\psi}^{[i]}+\boldsymbol{r}^{[i]} \wedge$ $\left(\boldsymbol{y}-\boldsymbol{y}_{B^{[i]}}\right)$ in $\Omega \times B^{[i]}$. Moreover, if $B_{\varepsilon}^{[i]}$ is a set of fibers parallel to $\boldsymbol{e}_{3}^{[i]}$, then $\boldsymbol{r}^{[i]}=\varphi^{[i]} \boldsymbol{e}_{3}^{[i]}$ for some $\varphi^{[i]} \in L^{2}(\Omega)$ and if in addition $\mu_{1 \varepsilon}^{[i]} \rightarrow+\infty$, then $\left(\boldsymbol{\psi}^{[i]} \cdot e_{3}^{[i]}\right)=\varphi^{[i]}=0$. The space $H$ is equipped with the inner product $\left(\boldsymbol{w}_{0}, \tilde{\boldsymbol{w}}_{0}\right)_{H}:=\int_{\Omega \times Y} \rho \boldsymbol{w}_{0} . \tilde{\boldsymbol{w}}_{0} d x d y$. The Hilbert space $V$ is the closure of $\mathcal{D}\left(\Omega ; C_{\sharp}^{\infty}(Y)\right) \cap H$ with respect to the norm $\|.\|_{V}$ defined by

$$
\left|\boldsymbol{w}_{0}\right|_{V}^{2}:=\left|\boldsymbol{w}_{0}\right|_{H}^{2}+\sum_{i=1}^{m} \bar{a}^{[i]}\left(\left(\boldsymbol{\psi}^{[i]}, \boldsymbol{r}^{[i]}\right),\left(\boldsymbol{\psi}^{[i]}, \boldsymbol{r}^{[i]}\right)\right)+\int_{\Omega \times(Y \backslash B)}\left|\nabla_{y} \boldsymbol{w}_{0}\right|^{2} d x d y
$$


The set $V$ is continuously embedded in $H$. The bilinear form $\bar{a}^{[i]}$ identical, up to a rotation of the coordinate axes, to one of the forms $\bar{a}, \bar{a}^{(2)}, \bar{a}^{(3)}, \bar{a}^{(4)}$ defined, depending on the order of magnitude of the elastic moduli and of the shape of the specified inclusions, by (7.22), (7.39), (7.45), (8.6) (if $\frac{\mu_{1 \varepsilon}^{i}}{\varepsilon^{2}} \rightarrow+\infty$, then $\bar{a}^{[i]}=0$ ). The symmetric bilinear form $a$ on $V$ is defined by

$$
a\left(\boldsymbol{w}_{0}, \boldsymbol{w}_{0}\right):=\int_{\Omega \times Y} \boldsymbol{e}_{y}\left(\boldsymbol{w}_{0}\right): \boldsymbol{\sigma}_{0 y}\left(\boldsymbol{w}_{0}\right) d x d y+\sum_{i=1}^{m} \bar{a}^{[i]}\left(\left(\boldsymbol{\psi}^{[i]}, \boldsymbol{r}^{[i]}\right),\left(\boldsymbol{\psi}^{[i]}, \boldsymbol{r}^{[i]}\right)\right)
$$

The Euler-Lagrange equations associated with (4.1) consist of a system of the type

$$
\left\{\begin{array}{l}
\left(\mathcal{P}_{\text {matrix }}^{\text {hom, multi }}\right), \\
\left(\mathcal{P}^{\text {hom } 1}\right), \\
\vdots \\
\left(\mathcal{P}^{\text {hom m }}\right),
\end{array}\right.
$$

of variables $\boldsymbol{v}^{[i]}, \boldsymbol{r}^{[i]}, \boldsymbol{u}_{0}$. The fields $\boldsymbol{v}^{[i]}$ and $\boldsymbol{r}^{[i]}$ characterize respectively the average effective displacement and the rescaled effective rotation vector in the inclusions $B_{\varepsilon}^{[i]}$. They are obtained as the weak-star limit in $L^{\infty}\left(0, T ; L^{2}\left(\Omega ; \mathbb{R}^{3}\right)\right)$ of the sequences $\boldsymbol{v}_{\varepsilon}^{[i]}$ and $\boldsymbol{r}_{\varepsilon}^{[i]}$ defined by substituting $B^{[i]}$ for $B$ in (2.16), (3.3). The effective displacement in the matrix is governed by $\left(\mathcal{P}_{\text {matrix }}^{\text {hom, multi }}\right)$. The system $\left(\mathcal{P}_{\text {matrix }}^{\text {hom, multi }}\right)$ differs from (2.18) only by its second line, namely

$$
\boldsymbol{u}_{0}=\boldsymbol{v}^{[i]}+\boldsymbol{r}^{[i]} \wedge\left(\boldsymbol{y}-\boldsymbol{y}_{B^{[i]}}\right) \text { in } \Omega \times(0, T) \times B^{[i]}, i \in\{1, \ldots, m\} .
$$

The system ( $\left.\mathcal{P}^{\text {hom }}{ }^{[i]}\right)$ governs the behavior of the effective displacement in $B_{\varepsilon}^{[i]}$. In the case of grain-like inclusions $\left(\mathcal{P}^{\text {hom }}[i]\right)$ is given by (3.6), being understood that all quantities defined in terms of $B$ (that is $\boldsymbol{v}, \boldsymbol{r}, \boldsymbol{g}, \boldsymbol{m}, \boldsymbol{y}_{G}, \boldsymbol{y}_{B}$, etc..) are now defined in terms of $B^{[i]}$ and labelled with the index [i]. If $B_{\varepsilon}^{[i]}$ consists of fibers parallel to $\boldsymbol{e}_{3}^{[i]}$, then $\boldsymbol{r}^{[i]}=\theta^{[i]} \boldsymbol{e}_{3}^{[i]}$ and $\left(\mathcal{P}^{\text {hom }}[i]\right)$ is a system of equations of $\left(\boldsymbol{v}^{[i]}, \theta^{[i]}\right)$ given in any orthonormal basis $\left(\boldsymbol{e}_{1}^{[i]}, \boldsymbol{e}_{2}^{[i]}, \boldsymbol{e}_{3}^{[i]}\right)$ by a system of the type (2.19), (2.20), (2.21), (2.22), according to the order of magnitude of $\mu_{1 \varepsilon}^{[i]}$.

5. Case of equilibrium equations.. In this section we complete and correct in the linear case the results obtained by the author with G. Bouchitté in [9] . The main novelty of our results in the elliptic case, compared to the results already available in [9] , concerns the case of fibers with elastic moduli of order 1 . Let $\boldsymbol{u}_{\varepsilon}$ be the solution of

$$
-\operatorname{div}\left(\boldsymbol{\sigma}_{\varepsilon}\left(\boldsymbol{u}_{\varepsilon}\right)\right)=\rho_{\varepsilon} \boldsymbol{f} \quad \text { in } \quad \Omega, \quad \boldsymbol{u}_{\varepsilon} \in H_{0}^{1}\left(\Omega, \mathbb{R}^{3}\right), \quad \boldsymbol{f} \in L^{2}\left(\Omega, \mathbb{R}^{3}\right) .
$$

Let $V$ and $H$ be the Hilbert spaces and let $a$ be the positive symmetric bilinear form defined in Section 4. By repeating the argument of the proofs of Theorem 2.1 and Theorem 3.1, we obtain: 
Corollary 5.1. Assume that $\boldsymbol{u}_{\varepsilon}$ two-scale converges, up to a subsequence, to some $\boldsymbol{u}_{0} \in L^{2}\left(\Omega \times Y ; \mathbb{R}^{3}\right)$. Then

$$
\boldsymbol{u}_{0} \in V \quad \text { and } \quad a\left(\boldsymbol{u}_{0}, \boldsymbol{w}_{0}\right)=\left(\boldsymbol{f}, \boldsymbol{w}_{0}\right)_{H}, \quad \forall \boldsymbol{w}_{0} \in V .
$$

When two-phase composites are considered, the effective problem (5.2) is in general ill-posed. More precisely, we show in the next proposition that in the two-phase case, unless very stiff fibers with elastic moduli of order greater than or equal to $\frac{1}{\varepsilon^{2}}$ are considered, the bilinear form $a$ fails to be coercive on $V$. Then, the problem (5.2) has no solution if $\boldsymbol{f}$ is not parallel to the fibers a.e., and has infinitely many solutions otherwise. In the former case, it follows from Corollary 5.1 that $\lim _{\varepsilon \rightarrow 0}\left|\boldsymbol{u}_{\varepsilon}\right|_{L^{2}\left(\Omega ; \mathbb{R}^{3}\right)}=+\infty$. Heuristically, this means that the effective composite can not "resist" to transverse body forces. In all likelihood, in the corresponding hyperbolic case, there holds $\lim _{T \rightarrow+\infty}|\boldsymbol{u}(T)|_{L^{2}\left(\Omega ; \mathbb{R}^{3}\right)}=+\infty$ for the same choice of $\boldsymbol{f}$ independent of $t$ (see Remark $5.3(\mathrm{v})$ ). This means that the effective composite "collapses". Hence both the elliptic model and the hyperbolic model seem to be unsatisfactory on a physical point of view when the bilinear form $a$ is not coercive on $V$ and when the body forces are not parallel a.e. to the fibers (see Remark 5.3 (iii)). In the following proposition, we state several necessary and sufficient conditions ensuring the coercivity of $a$ on $V$. The multiphase media satisfying these conditions (see Proposition $5.2(\mathrm{v})$ ) are likely to provide a physically relevant model of composite exhibiting torsion effects.

PROPOSITION 5.2. a) The following assertions are equivalent:

(i) The form a is coercive on $V$.

(ii) The problem (5.2) has a unique solution for all $\boldsymbol{f} \in L^{2}\left(\Omega ; \mathbb{R}^{3}\right)$.

(iii) The following estimate is satisfied:

$$
\int_{\Omega}|\boldsymbol{w}|^{2} d x \leq C F_{\varepsilon}(\boldsymbol{w}), \quad \forall \boldsymbol{w} \in H_{0}^{1}\left(\Omega ; \mathbb{R}^{3}\right) ; \quad F_{\varepsilon}(\boldsymbol{w}):=\frac{1}{2} \int_{\Omega} \boldsymbol{e}(\boldsymbol{w}): \boldsymbol{\sigma}_{\varepsilon}(\boldsymbol{w}) d x .
$$

(iv) For every $\boldsymbol{f} \in L^{2}\left(\Omega ; \mathbb{R}^{3}\right)$, the sequence $\left(\boldsymbol{u}_{\varepsilon}\right)$ has a bounded subsequence in $L^{2}(\Omega$; $\left.\mathbb{R}^{3}\right)$.

(v) One of the following conditions (a) or (b) is verified:

(a) the set of inclusions contains an $\varepsilon$-periodic distribution of parallel fibers with elastic moduli of order greater than or equal to $\frac{1}{\varepsilon^{2}}$,

(b) the set of inclusions contains three disconnected $\varepsilon$-periodic distributions of parallel fibers with elastic moduli of order greater than or equal to 1, distributed in three independent directions.

(vi) For every $\boldsymbol{f} \in L^{2}\left(\Omega ; \mathbb{R}^{3}\right)$, the sequence $\left(\boldsymbol{u}_{\varepsilon}\right)$ two-scale converges to the unique solution of (5.2).

b) If a is not coercive and if $\boldsymbol{f}$ does not belong a.e. to the subspace spanned by the directions of the fibers, then (5.2) has no solution.

Let us revisit now the example studied in [9] (Example 2) by G. Bouchitte and the author, who considered the case of a union of three non intersecting families of parallel fibers $B_{\varepsilon}=B_{\varepsilon}^{[1]} \cup B_{\varepsilon}^{[2]} \cup B_{\varepsilon}^{[3]}$, the fibers constituting $B_{\varepsilon}^{[i]}$ being parallel to $\boldsymbol{e}_{i}$ and having elastic moduli $\mu_{\varepsilon}^{[i]}$ of order 1 (that is $\left.\mu_{\varepsilon}^{[i]} \rightarrow k^{[i]} \in\right] 0,+\infty[$ ). Notice that the assertion (v) of Proposition 5.2 is satisfied, hence, by (vi), the sequence $\left(\boldsymbol{u}_{\varepsilon}\right)$ twoscale converges to the unique solution $\boldsymbol{u}_{0}$ of the problem (5.2). The Hilbert space $H$, equipped with the inner product $\left(\boldsymbol{w}_{0}, \tilde{\boldsymbol{w}}_{0}\right)_{H}:=\int_{\Omega \times Y} \rho \boldsymbol{w}_{0} . \tilde{\boldsymbol{w}}_{0} d x d y$, is the subset of $L^{2}\left(\Omega ; L_{\sharp}^{2}\left(Y ; \mathbb{R}^{3}\right)\right)$ consisting of those $\boldsymbol{w}_{0}$ such that for each $i \in\{1,2,3\}$ there exists a 
couple $\left(\boldsymbol{\psi}^{[i]}, \varphi^{[i]}\right) \in L^{2}\left(\Omega ; \mathbb{R}^{3}\right) \times L^{2}(\Omega)$ such that $\boldsymbol{w}_{0}=\boldsymbol{\psi}^{[i]}+\varphi^{[i]} \boldsymbol{e}_{i} \wedge\left(\boldsymbol{y}-\boldsymbol{y}_{B^{[i]}}\right)$ in $\Omega \times B^{[i]}$. The set $V$ is the closure of $\mathcal{D}\left(\Omega ; C_{\sharp}^{\infty}\left(Y ; \mathbb{R}^{3}\right)\right) \cap H$ with respect to the norm $|\cdot|_{V}$ defined by (4.2). The effective energy is defined on $V \times V$ by

$$
\begin{aligned}
& \frac{1}{2} a\left(\boldsymbol{w}_{0}, \boldsymbol{w}_{0}\right):=\frac{1}{2} \int_{\Omega \times Y} \boldsymbol{e}_{y}\left(\boldsymbol{w}_{0}\right): \boldsymbol{\sigma}_{0 y}\left(\boldsymbol{w}_{0}\right) d x d y+\frac{1}{2} \sum_{i=1}^{3} \bar{a}^{[i]}\left(\boldsymbol{w}_{0}, \boldsymbol{w}_{0}\right), \\
& \bar{a}^{[i]}\left(\boldsymbol{w}_{0}, \boldsymbol{w}_{0}\right):=k^{[i]} \int_{\Omega}\left|B^{[i]}\right| \frac{3 l+2}{l+1}\left|\frac{\partial \psi_{i}^{[i]}}{\partial x_{i}}\right|^{2} d x+\sum_{i=1}^{3} k^{[i]} \int_{\Omega} J^{[i]}\left|\frac{\partial \varphi^{[i]}}{\partial x_{i}}\right|^{2} d x .
\end{aligned}
$$

The second term of the right-hand side of the equation in the second line of (5.4) characterizes the torsional energy stored in the fibers. Formula (5.4) corrects in the linear case Formula (2.25) of [9], where the torsional terms are missing. As already said, the result stated in Theorem 2.4 of [9] is false. In fact, the crucial part of the proof of Theorem 2.4 of [9] was undone (see Remark 5.3 (iv)).

Remark 5.3. (i) Under the assumptions of Corollary 5.1 and the notations of Section 4 , the sequence $\left(\boldsymbol{v}_{\varepsilon}^{[i]}, \boldsymbol{r}_{\varepsilon}^{[i]}\right)$ converges weakly in $\left(L^{2}\left(\Omega ; \mathbb{R}^{3}\right)\right)^{2}$ to $\left(\boldsymbol{v}^{[i]}, \boldsymbol{r}^{[i]}\right)$ for each $i \in$ $\{1, \ldots, m\}$. The field $\boldsymbol{u}_{0}$ and the fields $\boldsymbol{v}^{[i]}, \boldsymbol{r}^{[i]}$ are solution of the system deduced formally from ( 4.4) by replacing the symbols of the type $\int_{A \times(0, T)} . . d m d t, L^{p}(0, T ; X)$, $\Omega \times(0, T), \boldsymbol{w}(\tau), \boldsymbol{w}(0), \frac{\partial \boldsymbol{w}}{\partial t}, \boldsymbol{a}_{0}, \boldsymbol{b}_{0} \ldots$ by $\int_{A} . . d m, X, \Omega, \boldsymbol{w}, 0,0,0,0 \ldots$ If ( 5.3) takes place and if the sequence $\left(\boldsymbol{u}_{0}\left(x, \frac{x}{\varepsilon}\right)\right)$ two-scale converges strongly to the solution $\boldsymbol{u}_{0}$ of the effective elliptic problem (see Remark 2.2 (iv)), then the corrector result $\lim _{\varepsilon \rightarrow 0}\left\|\boldsymbol{u}_{\varepsilon}-\boldsymbol{u}_{0}\left(x, \frac{x}{\varepsilon}\right)\right\|_{L^{2}\left(\Omega ; \mathbb{R}^{3}\right)}=0$ can be proved in similar manner as in the hyperbolic case.

(ii) In the elliptic case, the variable $\boldsymbol{u}_{0}$ can be eliminated in the effective problem: by the system of equations deduced from ( $\mathcal{P}_{\text {matrix }}^{\text {hom, multi }}$ ) as described in (i) and by Formula $\boldsymbol{u}=\int_{Y} \boldsymbol{u}_{0}(., y) d y$, we have, in the basis $\left(\boldsymbol{e}_{1}, \boldsymbol{e}_{2}, \boldsymbol{e}_{3}\right)$,

$$
\begin{aligned}
& \frac{1}{2} a\left(\boldsymbol{w}_{0}, \boldsymbol{w}_{0}\right):=\frac{1}{2} \int_{\Omega \times Y} \boldsymbol{e}_{y}\left(\boldsymbol{w}_{0}\right): \boldsymbol{\sigma}_{0 y}\left(\boldsymbol{w}_{0}\right) d x d y+\frac{1}{2} \sum_{i=1}^{3} \bar{a}^{[i]}\left(\boldsymbol{w}_{0}, \boldsymbol{w}_{0}\right), \\
& \boldsymbol{u}=\int_{Y} \boldsymbol{\gamma}_{0} d y+\sum_{i=1}^{m} \sum_{j=1}^{3} v_{j}^{[i]} \int_{Y} \boldsymbol{\xi}_{0}^{j[i]} d y+r_{j}^{[i]} \int_{Y} \boldsymbol{\eta}_{0}^{j[i]} d y,
\end{aligned}
$$

where $\boldsymbol{\xi}_{0}^{j[i]}, \boldsymbol{\eta}_{0}^{j[i]}, \boldsymbol{\gamma}_{0}(x,$.$) are the unique solution of$

$$
\begin{aligned}
&-\operatorname{div}_{y}\left(\boldsymbol{\sigma}_{0 y}\left(\boldsymbol{\xi}_{0}^{j[i]}\right)\right)=0 \text { in } Y \backslash B, \boldsymbol{\xi}_{0}^{j[i]}=\boldsymbol{e}_{j} \quad \text { in } B^{[i]}, \boldsymbol{\xi}_{0}^{j[i]}=0 \text { in } B \backslash B^{[i]}, \\
&-\operatorname{div}_{y}\left(\boldsymbol{\sigma}_{0 y}\left(\boldsymbol{\eta}_{0}^{j[i]}\right)\right)=0 \text { in } Y \backslash B, \boldsymbol{\eta}_{0}^{j[i]}=\boldsymbol{e}_{j} \wedge\left(\boldsymbol{y}-\boldsymbol{y}_{B^{[i]}}\right) \text { in } B^{[i]}, \boldsymbol{\eta}_{0}^{j[i]}=0 \text { in } B \backslash B^{[i]}, \\
&-\operatorname{div}_{y}\left(\boldsymbol{\sigma}_{0 y}\left(\boldsymbol{\gamma}_{0}\right)\right)=\rho \boldsymbol{f} \quad \text { in } \Omega \times(Y \backslash B), \quad \boldsymbol{\gamma}_{0}=0 \text { in } \Omega \times B, \\
& \boldsymbol{\sigma}_{0 y}\left(\boldsymbol{\zeta}_{0}\right) . \boldsymbol{n}(y)=-\boldsymbol{\sigma}_{0 y}\left(\boldsymbol{\zeta}_{0}\right) \cdot \boldsymbol{n}(-y) \text { on } \partial Y, \boldsymbol{\zeta}_{0} \in H_{\sharp}^{1}\left(Y ; \mathbb{R}^{3}\right), \\
& \boldsymbol{\zeta}_{0} \in\left\{\boldsymbol{\xi}_{0}^{j[i]}, \boldsymbol{\eta}_{0}^{j[i]}, \boldsymbol{\gamma}_{0}(x, .)\right\} .
\end{aligned}
$$


In the case of a two-phase composite (that is $m=1, B=B^{[1]}$ ), by (2.3), (5.6) and the Gauss-Green's Theorem there holds

$$
\begin{aligned}
& \boldsymbol{\xi}_{0}^{j}=\boldsymbol{e}_{j}, \quad \boldsymbol{g}\left(\boldsymbol{\xi}_{0}^{j}\right)=\boldsymbol{m}\left(\boldsymbol{\xi}_{0}^{j}\right)=\boldsymbol{g}\left(\boldsymbol{\eta}_{0}^{j}\right)=\boldsymbol{m}\left(\boldsymbol{\eta}_{0}^{j}\right)=0, \\
& \boldsymbol{g}\left(\boldsymbol{u}_{0}\right)=\boldsymbol{g}\left(\gamma_{0}\right)=\left(\int_{Y \backslash B} \rho d y\right) \boldsymbol{f}, \\
& \boldsymbol{m}\left(\boldsymbol{u}_{0}\right)=\boldsymbol{m}\left(\gamma_{0}\right)=\left(\int_{Y \backslash B} \rho\left(\boldsymbol{y}-\boldsymbol{y}_{B}\right) d y\right) \wedge \boldsymbol{f} .
\end{aligned}
$$

A similar computation can be done in the case of hyperbolic equations, when the mass density is supposed to vanish in the matrix, namely when $\rho 1_{Y \backslash B}=0$. Then the fields $\boldsymbol{u}_{0}$ and $\boldsymbol{u}$ are given in terms of the fields $\boldsymbol{v}^{[i]}$ and $\boldsymbol{r}^{[i]}(i \in\{1, \ldots, m\})$ simply by substituting 0 for $\gamma_{0}$ in (5.5). Under the same assumption, similar problems are tackled in [25] in the fibered case and in [26] in the case of grain-like inclusions, by using asymptotic expansions.

(iii) The case where $a$ is not coercive on $V$ and where $\boldsymbol{f}$ belongs a.e. to the subspace spanned by the directions of the fibers may have some interest on a physical level. However it seems difficult to find out in this case whether the sequence of the solutions of (5.1) is bounded in $L^{2}\left(\Omega ; \mathbb{R}^{3}\right)$ or not.

(iv) In [9], we have employed the $\Gamma$-convergence method, which is convenient for elliptic problems but not for hyperbolic problems, and which consists in establishing the convergence of the sequence of energy functionals $\left(F_{\varepsilon}\right)$ (see (5.3)), in some sense, to the effective energy $F(\boldsymbol{u}):=\inf \left\{\frac{1}{2} a\left(\boldsymbol{w}_{0}, \boldsymbol{w}_{0}\right), \boldsymbol{w}_{0} \in V, \int_{Y} \boldsymbol{w}_{0} d y=\boldsymbol{u}\right\}$. This approach allowed us to state our results in Example I and in Example III in the context of a simplified model of small deformation nonlinear elasticity. The crucial step in the $\Gamma$ convergence method is the so-called "upper bound" (see for intance [15] for all details relative to this notion of convergence). Our omission in Example II is that we did not check properly the proof of the "upper bound" and announced that this proof was the same as in Example I up to minor modifications (see [9], p.178,l.(-7) ), which is not true. Indeed, we have only established in Example II a lower bound for the effective energy.

(v) If a is coercive on $V$ and if $\boldsymbol{f}$ is independent of $t$, then it can be shown that the sequence $(\boldsymbol{u}(T))_{T>0}$ is bounded in $L^{2}\left(\Omega ; \mathbb{R}^{2}\right)$.

6. Preliminary results and a priori estimates.. The following section is devoted to the study, in the fibered case, of the asymptotic behavior of the sequence $\left(\boldsymbol{u}_{\varepsilon}\right)$ of the solutions of (1.1) and of the sequences $\left(\boldsymbol{v}_{\varepsilon}\right)$ and $\left(\theta_{\varepsilon}\right)$ defined by (2.16) (cf. Proposition 6.4). It includes also a technical lemma (Lemma 6.1) concerning the twoscale convergence and a theorem (Theorem 6.2) gathering some classical theoretical results about hyperbolic equations that will be employed to establish the well-posed nature of Problem (2.17) and the corrector result (2.25).

A fundamental property of the two-scale convergence (defined by $(2.5)$ ) is that any sequence bounded in $L^{2}\left(0, T ; L^{2}(\Omega)\right)$ admits a two-scale convergent subsequence. A sequence $\left(\varphi_{\varepsilon}\right) \subset L^{2}\left(0, T ; L^{2}(\Omega)\right)$ is said to be admissible if it two-scale converges to some $\varphi_{0} \in L^{2}\left(0, T ; L^{2}(\Omega \times Y)\right)$ and if, for every two-scale convergent sequence $\left(f_{\varepsilon}\right)$, there holds

$$
\lim _{\varepsilon \rightarrow 0} \int_{\Omega \times(0, T)} f_{\varepsilon} \varphi_{\varepsilon} d x d t=\int_{\Omega \times(0, T) \times Y} f_{0} \varphi_{0} d x d t d y
$$


It turns out that the set of all admissible sequences is equal to the set of all sequences $\left(\varphi_{\varepsilon}\right) \subset L^{2}\left(0, T ; L^{2}(\Omega)\right)$ satisfying $(2.6)$ for some $\varphi_{0} \in L^{2}\left(0, T ; L^{2}(\Omega \times Y)\right)$ (that is the set of all two-scale strongly convergent sequences). Indeed, the following implication is proved in [[2], Theorem 1.8]

$$
\begin{aligned}
& f_{\varepsilon} \longrightarrow f_{0} \quad \text { and } \quad \varphi_{\varepsilon} \longrightarrow \varphi_{0} \Rightarrow \\
& \lim _{\varepsilon \rightarrow 0} \int_{\Omega \times(0, T)} f_{\varepsilon} \varphi_{\varepsilon} d x d t=\int_{\Omega \times(0, T) \times Y} f_{0} \varphi_{0} d x d t d y .
\end{aligned}
$$

Conversely, if $\left(\varphi_{\varepsilon}\right)$ is admissible, one sees by substituting $\varphi_{\varepsilon}$ for $f_{\varepsilon}$ in (6.1) that $\left(\varphi_{\varepsilon}\right)$ is two-scale strongly convergent.

Lemma 6.1. (i) Let $h_{0} \in L^{\infty}\left(0, T ; L^{\infty}\left(\Omega, C_{\sharp}(Y)\right)\right) \cup L_{\sharp}^{\infty}(Y, C(\overline{\Omega \times(0, T)}))$ and let $h_{\varepsilon}(x, t):=h_{0}\left(x, t, \frac{x}{\varepsilon}\right)$. Then for every sequence $\left(\chi_{\varepsilon}\right) \subset L^{2}\left(0, T ; L^{2}(\Omega)\right)$ the following implications hold:

$$
\begin{array}{lll}
\chi_{\varepsilon} \longrightarrow \chi_{0} & \Longrightarrow & \chi_{\varepsilon} h_{\varepsilon} \longrightarrow \chi_{0} h_{0}, \\
\chi_{\varepsilon} \longrightarrow \chi_{0} & \Longrightarrow & \chi_{\varepsilon} h_{\varepsilon} \longrightarrow \chi_{0} h_{0} .
\end{array}
$$

(ii) If $\left(f_{\varepsilon}\right)$ is bounded in $L^{\infty}\left(0, T ; L^{2}(\Omega)\right)$ and two-scale converges to $f_{0}$, then $f_{0} \in$ $L^{\infty}\left(0, T ; L^{2}(\Omega \times Y)\right)$. If in addition $\left(f_{\varepsilon}\right)$ is bounded in $W^{1, \infty}\left(0, T ; L^{2}(\Omega)\right)$, then $f_{0} \in$ $W^{1, \infty}\left(0, T ; L^{2}(\Omega \times Y)\right)$ and $\left(\frac{\partial f_{\varepsilon}}{\partial t}\right)$ two-scale converges to $\frac{\partial f_{0}}{\partial t}$. Besides, if $f_{\varepsilon}(0) \longrightarrow a_{0}$, then $a_{0}=f_{0}(0)$ and $\left(f_{\varepsilon}(\tau)\right) \longrightarrow f_{0}(\tau), \forall \tau \in[0, T]$. Moreover, if $\left(\frac{\partial f_{\varepsilon}}{\partial t}\right) \longrightarrow \frac{\partial f_{0}}{\partial t}$ and $f_{\varepsilon}(0) \longrightarrow a_{0}$, then $\left(f_{\varepsilon}(\tau)\right) \longrightarrow f_{0}(\tau), \forall \tau \in[0, T]$.

Proof. (i) Assuming $\left(\chi_{\varepsilon}\right) \longrightarrow \chi_{0}$, we fix a sequence $\left(f_{\varepsilon}\right)$ bounded in $L^{2}(0, T$; $\left.L^{2}(\Omega)\right)$, a positive real $\eta>0$ and a function $\psi_{0} \in C\left(\overline{\Omega \times(0, T)}, C_{\sharp}(Y)\right)$ such that

$$
f_{\varepsilon} \longrightarrow f_{0}, \quad\left\|\chi_{0}-\psi_{0}\right\|_{L^{2}\left(0, T ; L^{2}(\Omega \times Y)\right)}<\eta .
$$

Since $h_{0} \psi_{0} \in L^{2}\left(0, T ; L^{2}\left(\Omega, C_{\sharp}^{\infty}(Y)\right)\right) \cup L_{\sharp}^{2}(Y, C(\overline{\Omega \times(0, T)}))$, the sequence $\left(h_{\varepsilon} \psi_{\varepsilon}\right)$ $\left(\psi_{\varepsilon}(x, t):=\psi_{0}\left(x, t, \frac{x}{\varepsilon}\right)\right)$ is admissible with respect to the two-scale convergence (see [2], Lemma 5.2, Corollary 5.4). Thanks to (6.5) and to the strong two-scale convergence of $\left(\chi_{\varepsilon}-\psi_{\varepsilon}\right)$ to $\chi_{0}-\psi_{0}$ we infer

$$
\begin{aligned}
& \limsup _{\varepsilon \rightarrow 0}\left|\int_{\Omega \times(0, T)} \chi_{\varepsilon} h_{\varepsilon} f_{\varepsilon} d x d t-\int_{\Omega \times(0, T) \times Y} \chi_{0} h_{0} f_{0} d x d t d y\right| \\
& \leq \limsup _{\varepsilon \rightarrow 0}\left|\int_{\Omega \times(0, T)} h_{\varepsilon}\left(\chi_{\varepsilon}-\psi_{\varepsilon}\right) f_{\varepsilon} d x d t\right| \\
& \quad+\limsup _{\varepsilon \rightarrow 0}\left|\int_{\Omega \times(0, T)} h_{\varepsilon} \psi_{\varepsilon} f_{\varepsilon} d x d t-\int_{\Omega \times(0, T) \times Y} \chi_{0} h_{0} f_{0} d x d t d y\right| \\
& \leq \limsup _{\varepsilon \rightarrow 0}\left\|h_{\varepsilon}\right\|_{L^{\infty}}\left\|\chi_{\varepsilon}-\psi_{\varepsilon}\right\|_{L^{2}}\left\|f_{\varepsilon}\right\|_{L^{2}}+\left|\int_{\Omega \times(0, T) \times Y} h_{0}\left(\psi_{0}-\chi_{0}\right) f_{0} d x d t d y\right| \\
& \leq C \eta,
\end{aligned}
$$


hence $\chi_{\varepsilon} h_{\varepsilon} \longrightarrow \chi_{0} h_{0}$. Supposing now $\left(\chi_{\varepsilon}\right) \longrightarrow \chi_{0}$, fixing a sequence $\left(\varphi_{\varepsilon}\right)$ such that $\left(\varphi_{\varepsilon}\right) \longrightarrow \varphi_{0}$, we deduce from (6.3) that $\left(\varphi_{\varepsilon} h_{\varepsilon}\right) \longrightarrow \varphi_{0} h_{0}$, thus $\lim _{\varepsilon \rightarrow 0} \int_{\Omega \times(0, T)} \chi_{\varepsilon} h_{\varepsilon} \varphi_{\varepsilon} d x d t=$ $\int_{\Omega \times(0, T) \times Y} \chi_{0} h_{0} \varphi_{0} d x d t d y$. (ii) If $\left(f_{\varepsilon}\right)$ is bounded in $L^{\infty}\left(0, T ; L^{2}(\Omega)\right)$ and two-scale converges to $f_{0}$, fixing $\varphi_{0} \in C\left(\overline{\Omega \times(0, T)}, C_{\sharp}(Y)\right)$ and setting $\varphi_{\varepsilon}(x, t):=\varphi_{0}\left(x, t, \frac{x}{\varepsilon}\right)$, noticing that

$$
\begin{aligned}
\int_{\Omega \times(0, T)} f_{\varepsilon} \varphi_{\varepsilon} d x d t & \leq \int_{0}^{T}\left\|f_{\varepsilon}(., t)\right\|_{L^{2}(\Omega)}\left\|\varphi_{\varepsilon}(., t)\right\|_{L^{2}(\Omega)} d t \\
& \leq C \int_{0}^{T}\left\|\varphi_{\varepsilon}(., t)\right\|_{L^{2}(\Omega)} d t
\end{aligned}
$$

and that $\lim _{\varepsilon \rightarrow 0}\left\|\varphi_{\varepsilon}(., t)\right\|_{L^{2}(\Omega)}=\left\|\varphi_{0}(., t)\right\|_{L^{2}(\Omega \times Y)}, \forall t \in(0, T)$, by passing to the limit as $\varepsilon \rightarrow 0$ in (6.6) in accordance with (6.2) and the Dominated Convergence Theorem we infer

$$
\int_{\Omega \times(0, T) \times Y} f_{0} \varphi_{0} d x d t d y \leq C\left\|\varphi_{0}\right\|_{L^{1}\left(0, T, L^{2}(\Omega \times Y)\right)}, \quad \forall \varphi_{0} \in C\left(\overline{\Omega \times(0, T)}, C_{\sharp}(Y)\right),
$$

hence $f_{0} \in L^{\infty}\left(0, T ; L^{2}(\Omega \times Y)\right)$. If in addition $\left(\frac{\partial f_{\varepsilon}}{\partial t}\right)$ is bounded in $L^{\infty}\left(0, T ; L^{2}(\Omega)\right)$, by the same argument $\left(\frac{\partial f_{\varepsilon}}{\partial t}\right)$ two-scale converges up to a subsequence to some $\xi_{0} \in$ $L^{\infty}\left(0, T ; L^{2}(\Omega \times Y)\right)$, thus

$$
\begin{aligned}
\int_{\Omega \times(0, T) \times Y} \xi_{0} \psi_{0} d x d t d y & =\lim _{\varepsilon \rightarrow 0} \int_{\Omega \times(0, T)} \frac{\partial f_{\varepsilon}}{\partial t} \psi_{0}\left(x, t, \frac{x}{\varepsilon}\right) d x d t \\
& =-\lim _{\varepsilon \rightarrow 0} \int_{\Omega \times(0, T)} f_{\varepsilon} \frac{\partial \psi_{0}}{\partial t}\left(x, t, \frac{x}{\varepsilon}\right) d x d t \\
& =-\int_{\Omega \times(0, T) \times Y} f_{0} \frac{\partial \psi_{0}}{\partial t} d x d t d y, \quad \forall \psi_{0} \in \mathcal{D}\left(\Omega \times(0, T) ; C_{\sharp}^{\infty}(Y)\right) .
\end{aligned}
$$

Hence $\frac{\partial f_{0}}{\partial t}=\xi_{0}, f_{0} \in W^{1, \infty}\left(0, T ; L^{2}(\Omega \times Y)\right)$, and the convergence holds for the whole sequence. If $f_{\varepsilon}(0) \longrightarrow a_{0}$, fixing $\tau \in[0, T]$ and an admissible sequence $\left(\varphi_{\varepsilon}\right) \subset L^{2}(\Omega)$ such that $\left(\varphi_{\varepsilon}\right) \longrightarrow \varphi_{0} \in L^{2}(\Omega \times Y)$ and applying (6.4) with $h_{0}(x, t, y):=1_{[0, \tau]}(t)$, we obtain

$$
\begin{aligned}
\lim _{\varepsilon \rightarrow 0} \int_{\Omega} f_{\varepsilon}(\tau) \varphi_{\varepsilon} d x & =\lim _{\varepsilon \rightarrow 0} \int_{\Omega}\left(\int_{0}^{T} \frac{\partial f_{\varepsilon}}{\partial t} 1_{[0, \tau]}(t) d t+f_{\varepsilon}(0)\right) \varphi_{\varepsilon} d x \\
& =\int_{\Omega \times(0, T) \times Y} \frac{\partial f_{0}}{\partial t}(t) 1_{[0, \tau]}(t) \varphi_{0} d x d t d y+\int_{\Omega \times Y} a_{0} \varphi_{0} d x d y \\
& =\int_{\Omega \times Y}\left(f_{0}(\tau)-f_{0}(0)+a_{0}\right) \varphi_{0} d x d y,
\end{aligned}
$$

hence $f_{\varepsilon}(\tau) \longrightarrow f_{0}(\tau)-f_{0}(0)+a_{0}, \forall \tau \in[0, T]$. Fixing $\left.\varphi_{0} \in \mathcal{D}\left(\Omega \times(0, T) ; C_{\sharp}^{\infty}(Y)\right)\right)$, setting $\varphi_{\varepsilon}(x, t):=\varphi_{0}\left(x, t, \frac{x}{\varepsilon}\right)$ and applying the Dominated Convergence Theorem, we 
infer

$$
\begin{gathered}
\int_{\Omega \times(0, T) \times Y} f_{0} \varphi_{0} d x d y d t=\lim _{\varepsilon \rightarrow 0} \int_{\Omega \times(0, T)} f_{\varepsilon} \varphi_{\varepsilon} d x d t=\int_{0}^{T} \lim _{\varepsilon \rightarrow 0}\left(\int_{\Omega} f_{\varepsilon}(\tau) \varphi_{\varepsilon}(\tau) d x\right) d t \\
\left.=\int_{\Omega \times(0, T) \times Y}\left(f_{0}-f_{0}(0)+a_{0}\right) \varphi_{0} d x d t d y, \quad \forall \varphi_{0} \in \mathcal{D}\left(\Omega \times(0, T) ; C_{\sharp}^{\infty}(Y)\right)\right),
\end{gathered}
$$

hence $f_{0}(0)=a_{0}$. If $f_{\varepsilon}(0) \longrightarrow a_{0}$ and $\frac{\partial f_{\varepsilon}}{\partial t} \longrightarrow \frac{\partial f_{0}}{\partial t}$, we deduce from the previous reasoning that $f_{0}(0)=a_{0}$ and notice that $(6.8)$ holds for any two-scale converging sequence $\left(\varphi_{\varepsilon}\right)$.

The abstract results collected in the next theorem are proved in [19] (see Theorem 8.1 p. 287, Theorem 8.2 and Lemma 8.3 p. 298), [16] (see Formula (5.20) p. 667, and Theorem 1 p. 670), [18] (see Remark 1.3 p. 155). Henceforth, the derivatives in $\mathcal{D}^{\prime}(0, T ; H)$ are identified with the time derivatives in $\mathcal{D}^{\prime}(\Omega \times(0, T) \times Y)$ and are denoted both by $\frac{\partial \zeta}{\partial t}$ or by $\zeta^{\prime}$.

THEOREM 6.2. Let $V$ and $H$ be separable Hilbert spaces such that $V \subset H=$ $H^{\prime} \subset V^{\prime}$, with continuous and dense imbeddings. Let $\|.\|_{V},|\cdot|_{H},((., .))_{V},(., .)_{H}$ denote their respective norm and inner product. Let $a: V \times V \rightarrow \mathbb{R}$ be a continuous bilinear symmetric form on $V$. Let $A \in \mathcal{L}\left(V, V^{\prime}\right)$ be defined by $a(\xi, \tilde{\xi})=$ $(A \xi, \tilde{\xi})_{\left(V^{\prime}, V\right)}, \forall(\xi, \tilde{\xi}) \in V^{2}$. Assume that

$$
\exists(\lambda, \alpha) \in \mathbb{R}_{+} \times \mathbb{R}_{+}^{*}, \quad a(\xi, \xi)+\lambda|\xi|_{H}^{2} \geq \alpha\|\xi\|_{V}^{2}, \forall \xi \in V .
$$

Let $h \in L^{2}(0, T ; H), \xi_{0} \in V, \xi_{1} \in H$. Then there exists a unique solution $\xi$ of

$$
\begin{aligned}
& A \xi(t)+\xi^{\prime \prime}(t)=h(t), \quad \xi \in L^{2}(0, T ; V), \\
& \xi^{\prime} \in L^{2}(0, T ; H), \quad \xi(0)=\xi_{0}, \quad \xi^{\prime}(0)=\xi_{1},
\end{aligned}
$$

where $\xi^{\prime}=\frac{\partial \xi}{\partial t}, \xi^{\prime \prime}=\frac{\partial^{2} \xi}{\partial t^{2}}$. What is more,

$$
\xi \in C([0, T] ; V) \cap C^{1}([0, T] ; H), \quad \xi^{\prime} \in L^{2}(0, T ; V), \quad \xi^{\prime \prime} \in L^{2}\left(0, T ; V^{\prime}\right) .
$$

Besides, setting $e(\tau):=\frac{1}{2}\left[\left(\xi^{\prime}(\tau), \xi^{\prime}(\tau)\right)_{H}+a(\xi(\tau), \xi(\tau))\right], \forall \tau \in[0, T]$, there holds

$$
e(\tau)=e(0)+\int_{0}^{\tau}\left(h, \xi^{\prime}\right)_{H} d t, \quad \forall \tau \in[0, T]
$$

Moreover, Problem (6.10) is equivalent to

$$
\begin{gathered}
\int_{0}^{T}\left(a(\xi(t), \tilde{\xi}) \eta(t)+(\xi(t), \tilde{\xi})_{H} \eta^{\prime \prime}(t)\right) d t+\left(\xi_{0}, \tilde{\xi}\right)_{H} \eta^{\prime}(0) \\
-\left(\xi_{1}, \tilde{\xi}\right)_{H} \eta(0)=\int_{0}^{T}(h, \tilde{\xi})_{H} \eta(t) d t, \\
\forall \tilde{\xi} \in V, \quad \forall \eta \in \mathcal{D}(]-\infty, T[) ; \quad \xi \in L^{2}(0, T ; V), \xi^{\prime} \in L^{2}(0, T ; H) .
\end{gathered}
$$

The next lemma concerns both the fibered case and the case of grain-like particles. The estimate (6.14) will be employed in the demonstration of Proposition 6.4 as a 
means to prove the boundedness of the sequence $\left(\boldsymbol{u}_{\varepsilon}\right)$ of the solutions of (1.1) and also in Section 7, in order to establish the corrector result (2.25).

LEMma 6.3. Under the assumptions (2.7) and either (2.1) or (3.1), there holds

$$
\begin{aligned}
& \text { if } \inf _{B_{\varepsilon}} \rho_{\varepsilon}>c>0 \quad \text { or } \quad \inf _{\Omega \backslash B_{\varepsilon}} \rho_{\varepsilon}>c>0, \text { then } \\
& \begin{aligned}
& \int_{\Omega}|\boldsymbol{w}|^{2}(\tau) d x \leq C \int_{\Omega \times(0, T)} \rho_{\varepsilon}\left|\frac{\partial \boldsymbol{w}}{\partial t}\right|^{2} d x d t \\
&+C \int_{\Omega} \varepsilon^{2}|e(\boldsymbol{w})|^{2}(\tau) d x+C \int_{\Omega}|\boldsymbol{w}|^{2}(0) d x, \\
& \forall \tau \in[0, T], \quad \forall \boldsymbol{w} \in C\left([0, T] ; H_{0}^{1}\left(\Omega ; \mathbb{R}^{3}\right)\right) \cap C^{1}\left([0, T] ; L^{2}\left(\Omega ; \mathbb{R}^{3}\right)\right) .
\end{aligned}
\end{aligned}
$$

Proof. For each $w \in L^{2}(\Omega)$ we define, setting $w=0$ in $\Omega \backslash \mathbb{R}^{3}$,

$$
\widehat{w}_{\varepsilon}(x):=\sum_{i \in J_{\varepsilon}}\left(f_{B_{\varepsilon}^{i}} w d s\right) 1_{Y_{\varepsilon}^{i}}(x), \quad J_{\varepsilon}:=\left\{i \in \mathbb{Z}^{3}, Y_{\varepsilon}^{i} \cap \Omega \neq \emptyset\right\} .
$$

By making suitable changes of variables in the Poincaré-Wirtinger inequality $\int_{Y} \mid w$ $-\left.\left(f_{B} w d s\right)\right|^{2} d x \leq C \int_{Y}|\nabla w|^{2} d x, \forall w \in H^{1}(Y)$, we infer that $\int_{\Omega}\left|\boldsymbol{w}-\widehat{\boldsymbol{w}}_{\varepsilon}\right|^{2} d x \leq$ $C \int_{\Omega} \varepsilon^{2}|\boldsymbol{\nabla}(\boldsymbol{w})|^{2} d x, \forall \boldsymbol{w} \in H^{1}\left(\Omega ; \mathbb{R}^{3}\right)$. Therefore, by Korn's inequality in $H_{0}^{1}\left(\Omega ; \mathbb{R}^{3}\right)$ we have

$$
\int_{\Omega}\left|\boldsymbol{w}-\widehat{\boldsymbol{w}}_{\varepsilon}\right|^{2} d x \leq C \int_{\Omega} \varepsilon^{2}|\boldsymbol{e}(\boldsymbol{w})|^{2} d x, \forall \boldsymbol{w} \in H_{0}^{1}\left(\Omega, \mathbb{R}^{3}\right) .
$$

By (6.15) there holds $\int_{\Omega}\left|\widehat{\boldsymbol{w}}_{\varepsilon}\right|^{2} d x \leq C \int_{B_{\varepsilon}}|\boldsymbol{w}|^{2} d x, \forall \boldsymbol{w} \in L^{2}\left(\Omega ; \mathbb{R}^{3}\right)$, hence we infer from $(6.16)$

$$
\int_{\Omega}|\boldsymbol{w}|^{2} d x \leq C \varepsilon^{2} \int_{\Omega}|\boldsymbol{e}(\boldsymbol{w})|^{2} d x+C \int_{B_{\varepsilon}}|\boldsymbol{w}|^{2} d x, \forall \boldsymbol{w} \in H_{0}^{1}\left(\Omega, \mathbb{R}^{3}\right) .
$$

If $\inf _{B_{\varepsilon}} \rho_{\varepsilon}>c>0$, then

$$
\begin{aligned}
& \int_{B_{\varepsilon}}|\boldsymbol{w}|^{2}(\tau) d x= \int_{B_{\varepsilon}}\left|\int_{0}^{\tau} \frac{\partial \boldsymbol{w}}{\partial t}(s) d s+\boldsymbol{w}(0)\right|^{2} d x \\
& \leq C \int_{\Omega \times(0, T)} \rho_{\varepsilon}\left|\frac{\partial \boldsymbol{w}}{\partial t}\right|^{2} d x d t+C \int_{\Omega}|\boldsymbol{w}|^{2}(0) d x \\
& \\
& \quad \forall \boldsymbol{w} \in C\left([0, T] ; H_{0}^{1}\left(\Omega ; \mathbb{R}^{3}\right)\right) \cap C^{1}\left([0, T] ; L^{2}\left(\Omega ; \mathbb{R}^{3}\right)\right) .
\end{aligned}
$$

Assertion (6.14) follows then from (6.17) and (6.18). Otherwise, if $\inf _{\Omega \backslash B_{\varepsilon}} \rho_{\varepsilon}>c>0$, we repeat the same argument, substituting $Y \backslash B$ for $Y$.

The following proposition specifies, in the fibered case, the asymptotic behavior of several sequences associated to the sequence $\left(\boldsymbol{u}_{\varepsilon}\right)$ of the solutions of (1.1).

Proposition 6.4. There exists a unique solution $\boldsymbol{u}_{\varepsilon}$ of (1.1). Moreover, 


$$
\frac{\partial \boldsymbol{u}_{\varepsilon}}{\partial t} \in L^{2}\left(0, T ; H_{0}^{1}\left(\Omega ; \mathbb{R}^{3}\right)\right), \quad \frac{\partial^{2} \boldsymbol{u}_{\varepsilon}}{\partial t^{2}} \in L^{2}\left(0, T ; H^{-1}\left(\Omega ; \mathbb{R}^{3}\right)\right) .
$$

Under (2.8), (2.10), there exists a constant $C>0$ such that

$$
\begin{array}{lr}
\int_{\Omega \backslash B_{\varepsilon}} \varepsilon^{2}\left|\boldsymbol{e}\left(\boldsymbol{u}_{\varepsilon}\right)(\tau)\right|^{2} d x+\int_{\Omega}\left(\rho_{\varepsilon}\left|\frac{\partial \boldsymbol{u}_{\varepsilon}}{\partial t}\right|^{2}+\left|\boldsymbol{u}_{\varepsilon}\right|^{2}+\left|\boldsymbol{v}_{\varepsilon}\right|^{2}+\left|\theta_{\varepsilon}\right|^{2}\right) & (\tau) d x \leq C, \\
\int_{B_{\varepsilon}}\left|\boldsymbol{e}\left(\boldsymbol{u}_{\varepsilon}\right)(\tau)\right|^{2} d x+\int_{\Omega}\left|\frac{\partial v_{\varepsilon 3}}{\partial x_{3}}(\tau)\right|^{2} d x \leq \frac{C}{\mu_{1 \varepsilon}}, & \forall \tau \in[0, T], \\
\int_{\Omega}\left|v_{\varepsilon 1}(\tau)\right|^{2}+\left|v_{\varepsilon 2}(\tau)\right|^{2}+\left|\frac{v_{\varepsilon 3}}{\varepsilon}(\tau)\right|^{2} d x \leq \frac{C}{\varepsilon^{2} \mu_{1 \varepsilon}}, & \forall \tau \in[0, T],
\end{array}
$$

and fields $\boldsymbol{u}_{0} \in L^{\infty}\left(0, T ; L^{2}\left(\Omega \times Y ; \mathbb{R}^{3}\right)\right), \boldsymbol{u}, \boldsymbol{v} \in L^{\infty}\left(0, T ; L^{2}\left(\Omega ; \mathbb{R}^{3}\right)\right), \theta \in L^{\infty}(0, T ;$ $\left.L^{2}(\Omega)\right), \boldsymbol{\Xi}^{m}, \boldsymbol{\Xi}^{f} \in L^{\infty}\left(0, T ; L^{2}\left(\Omega \times Y ; \mathbb{S}^{3}\right)\right)$, such that, up to a subsequence,

$$
\begin{aligned}
& \boldsymbol{u}_{\varepsilon} \rightarrow \boldsymbol{u}_{0}, \quad \varepsilon \boldsymbol{e}\left(\boldsymbol{u}_{\varepsilon}\right) 1_{\Omega \backslash B_{\varepsilon}} \uplus \boldsymbol{\Xi}^{m}, \quad \boldsymbol{e}\left(\boldsymbol{u}_{\varepsilon}\right) 1_{B_{\varepsilon}} \uplus \boldsymbol{\Xi}^{f}, \\
& \boldsymbol{u}_{\varepsilon} \stackrel{\star}{*} \boldsymbol{u}, \boldsymbol{v}_{\varepsilon} \stackrel{\star}{*} \boldsymbol{v}, \theta_{\varepsilon} \stackrel{\star}{*} \theta, \frac{\partial v_{\varepsilon}}{\partial x_{3}} \stackrel{\star}{*} \frac{\partial v_{3}}{\partial x_{3}} \text { star-weakly in } L^{\infty}\left(0, T ; L^{2}\right) .
\end{aligned}
$$

Besides the next relations are satisfied

$$
\begin{aligned}
& \boldsymbol{u}_{0} \in L^{\infty}\left(0, T ; L^{2}\left(\Omega ; H_{\sharp}^{1}\left(Y ; \mathbb{R}^{3}\right)\right), \quad v_{3} \in L^{\infty}\left(0, T ; L^{2}\left(\omega ; H_{0}^{1}(0, L)\right)\right),\right. \\
& \boldsymbol{u}_{0}=\boldsymbol{v}+\theta \boldsymbol{e}_{3} \wedge\left(\boldsymbol{y}-\boldsymbol{y}_{B}\right) \quad \text { in } \Omega \times(0, T) \times B, \quad \boldsymbol{u}=\int_{Y} \boldsymbol{u}_{0}(., y) d y, \\
& \boldsymbol{v}=f_{B} \boldsymbol{u}_{0}(., y) d y, \quad \theta=\frac{1}{J} \int_{B} \boldsymbol{u}_{0}(., y) .\left(\boldsymbol{e}_{3} \wedge\left(\boldsymbol{y}-\boldsymbol{y}_{B}\right)\right) d y, \\
& \boldsymbol{\Xi}^{m}=\boldsymbol{e}_{y}\left(\boldsymbol{u}_{0}\right), \quad \boldsymbol{\Xi}^{f}=0 \quad \text { in } \Omega \times(0, T) \times Y \backslash B, \\
& \frac{\partial v_{3}}{\partial x_{3}}=\int_{-\frac{1}{2}}^{\frac{1}{2}} \Xi_{33}^{f} d y_{3} \quad \text { in } \Omega \times(0, T) \times B, \\
& \frac{\partial \theta}{\partial x_{3}}=\frac{2}{J}\left(\int_{B} \boldsymbol{\Xi}^{f} .\left(\boldsymbol{e}_{3} \wedge\left(\boldsymbol{y}-\boldsymbol{y}_{B}\right)\right) d y\right)_{3} \text { in } \Omega \times(0, T), \\
& \theta \in L^{\infty}\left(0, T ; L^{2}\left(\omega ; H_{0}^{1}(0, L)\right)\right),
\end{aligned}
$$

the last two lines of (6.22) being obtained under the additional assumption (2.13). Moreover,

$$
\theta=v_{3}=0 \quad \text { if } \quad k=+\infty, \quad \boldsymbol{v}=0 \quad \text { if } \quad \kappa=+\infty .
$$

If $\kappa \in] 0,+\infty]$, there exists $\zeta_{0} \in L^{\infty}\left(0, T ; L^{2}\left(\omega \times Y ; H_{0}^{1}(0, L)\right)\right), \Xi^{b} \in L^{\infty}(0, T$; $\left.L^{2}\left(\Omega \times Y ; \mathbb{S}^{3}\right)\right), \xi \in L^{\infty}\left(0, T ; L^{2}\left(\omega ; H_{0}^{1}(0, L)\right)\right)$ such that up to a subsequence,

$$
\frac{u_{\varepsilon 3}}{\varepsilon} 1_{B_{\varepsilon}} \longrightarrow \zeta_{0}, \quad \quad \frac{1}{\varepsilon} \boldsymbol{e}\left(\boldsymbol{u}_{\varepsilon}\right) 1_{B_{\varepsilon}} \longrightarrow \boldsymbol{\Xi}^{b}
$$


and

$$
\begin{aligned}
& v_{1}, v_{2} \in L^{\infty}\left(0, T ; L^{2}\left(\omega ; H_{0}^{2}(0, L)\right)\right), \\
& \zeta_{0}=\xi-\sum_{\alpha=1}^{2} \frac{\partial v_{\alpha}}{\partial x_{3}}\left(\boldsymbol{y}-\boldsymbol{y}_{B}\right)_{\alpha} \quad \text { in } \Omega \times(0, T) \times B, \\
& \int_{-\frac{1}{2}}^{\frac{1}{2}} \Xi_{33}^{b} d y_{3}=\frac{\partial \zeta_{0}}{\partial x_{3}}=\frac{\partial \xi}{\partial x_{3}}-\sum_{\alpha=1}^{2} \frac{\partial^{2} v_{\alpha}}{\partial x_{3}^{2}}\left(\boldsymbol{y}-\boldsymbol{y}_{B}\right)_{\alpha} \quad \text { in } \Omega \times(0, T) \times B .
\end{aligned}
$$

Under the additional hypothesis (2.14), we have for any $k \in] 0,+\infty]$,

$$
\begin{aligned}
& \boldsymbol{u}_{0} \in W^{1, \infty}\left(0, T ; L^{2}\left(\Omega \times Y ; \mathbb{R}^{3}\right)\right), \\
& \frac{\partial \boldsymbol{u}_{\varepsilon}}{\partial t} \longrightarrow \frac{\partial \boldsymbol{u}_{0}}{\partial t}, \quad \boldsymbol{u}_{\varepsilon}(\tau) \longrightarrow \boldsymbol{u}_{0}(\tau), \forall \tau \in[0, T]
\end{aligned}
$$

Proof. The problem (1.1) is equivalent to (6.13), where $H:=L^{2}\left(\Omega ; \mathbb{R}^{3}\right),(\boldsymbol{\xi}, \tilde{\boldsymbol{\xi}})_{H}:=$ $\int_{\Omega} \rho_{\varepsilon} \boldsymbol{\xi} \cdot \tilde{\boldsymbol{\xi}} d x, V:=H_{0}^{1}\left(\Omega ; \mathbb{R}^{3}\right)\left(V^{\prime}=H^{-1}\left(\Omega ; \mathbb{R}^{3}\right)\right), a(\boldsymbol{\xi}, \tilde{\boldsymbol{\xi}}):=\int_{\Omega} \boldsymbol{\sigma}_{\varepsilon}(\boldsymbol{\xi}): \boldsymbol{e}(\tilde{\boldsymbol{\xi}}) d x$, $\left(\boldsymbol{\xi}_{0}, \boldsymbol{\xi}_{1}, \boldsymbol{h}\right)=\left(\boldsymbol{a}_{0}, \boldsymbol{b}_{0}, \boldsymbol{f}\right)$. By (2.14) and (2.15), $H$ is a Hilbert space and the assumptions of Theorem 6.2 are satisfied. Therefore (1.1) has a unique solution and (6.19) follows from (6.11). By (6.12) we have, for all $\tau \in[0, T]$,

$$
\begin{aligned}
\frac{1}{2} \int_{\Omega}\left(\rho_{\varepsilon}\left|\frac{\partial \boldsymbol{u}_{\varepsilon}}{\partial t}\right|^{2}+\boldsymbol{\sigma}_{\varepsilon}\left(\boldsymbol{u}_{\varepsilon}\right): \boldsymbol{e}\left(\boldsymbol{u}_{\varepsilon}\right)\right)(\tau) d x \\
\quad=\frac{1}{2} \int_{\Omega}\left(\rho_{\varepsilon}\left|\boldsymbol{b}_{0}\right|^{2}+\boldsymbol{\sigma}_{\varepsilon}\left(\boldsymbol{a}_{0}\right): \boldsymbol{e}\left(\boldsymbol{a}_{0}\right)\right) d x+\int_{\Omega \times(0, \tau)} \rho_{\varepsilon} \boldsymbol{f} \cdot \frac{\partial \boldsymbol{u}_{\varepsilon}}{\partial t} d x d t
\end{aligned}
$$

By (2.10) there holds $\int_{\Omega}\left(\rho_{\varepsilon}\left|\boldsymbol{b}_{0}\right|^{2}+\boldsymbol{\sigma}_{\varepsilon}\left(\boldsymbol{a}_{0}\right): \boldsymbol{e}\left(\boldsymbol{a}_{0}\right)\right) d x+\int_{\Omega \times(0, T)} \rho_{\varepsilon}|\boldsymbol{f}|^{2} d x d t \leq C$, hence

$$
\begin{aligned}
\int_{\Omega}\left(\rho_{\varepsilon}\left|\frac{\partial \boldsymbol{u}_{\varepsilon}}{\partial t}\right|^{2} d x+\sigma_{\varepsilon}\left(\boldsymbol{u}_{\varepsilon}\right): \boldsymbol{e}\left(\boldsymbol{u}_{\varepsilon}\right)\right)(\tau) d x \\
\leq C\left(1+\sqrt{\int_{\Omega \times(0, T)} \rho_{\varepsilon}\left|\frac{\partial \boldsymbol{u}_{\varepsilon}}{\partial t}\right|^{2} d x d t}\right), \forall \tau \in[0, T] .
\end{aligned}
$$

By integrating (6.28) with respect to $\tau$ over $(0, T)$, we deduce that $\int_{\Omega \times(0, T)} \rho_{\varepsilon}\left|\frac{\partial \boldsymbol{u}_{\varepsilon}}{\partial t}\right|^{2}$ $d x d t \leq C$ and then, coming back to (6.28), that

$$
\int_{\Omega} \rho_{\varepsilon}\left|\frac{\partial \boldsymbol{u}_{\varepsilon}}{\partial t}\right|^{2}(\tau) d x+\int_{\Omega} \boldsymbol{\sigma}_{\varepsilon}\left(\boldsymbol{u}_{\varepsilon}\right): \boldsymbol{e}\left(\boldsymbol{u}_{\varepsilon}\right)(\tau) d x \leq C, \quad \forall \tau \in[0, T] .
$$

We infer from (1.1), (2.8), (2.16), (6.29) that

$$
\begin{aligned}
\int_{\Omega} \rho_{\varepsilon}\left|\frac{\partial \boldsymbol{u}_{\varepsilon}}{\partial t}\right|^{2}(\tau)+\varepsilon^{2}\left|\boldsymbol{e}\left(\boldsymbol{u}_{\varepsilon}\right)\right|^{2}(\tau) d x \leq C, \\
\int_{B_{\varepsilon}}\left|e\left(\boldsymbol{u}_{\varepsilon}\right)\right|^{2}(\tau) d x+\int_{\Omega}\left|\frac{\partial v_{\varepsilon 3}}{\partial x_{3}}\right|^{2}(\tau) d x \leq \frac{C}{\mu_{1 \varepsilon}} .
\end{aligned}
$$


By (6.17) and by the inequality (see [9], Formula (4.32))

$$
(6.31) \int_{B_{\varepsilon}}\left(\left|w_{1}\right|^{2}+\left|w_{2}\right|^{2}+\left|\frac{w_{3}}{\varepsilon}\right|^{2}\right) d x \leq \frac{C}{\varepsilon^{2}} \int_{B_{\varepsilon}}|\boldsymbol{e}(\boldsymbol{w})|^{2} d x, \quad \forall \boldsymbol{w} \in H_{0}^{1}\left(\Omega ; \mathbb{R}^{3}\right),
$$

deduced by making appropriate changes of variables in the Korn's inequality $\int_{B}|\boldsymbol{w}|^{2} d x$ $\leq C \int_{B}|\boldsymbol{e}(\boldsymbol{w})|^{2} d x, \forall \boldsymbol{w} \in\left\{\boldsymbol{\xi} \in H^{1}\left(B ; \mathbb{R}^{3}\right), \boldsymbol{\xi}\left(y_{1}, y_{2},-\frac{1}{2}\right)=0\right\}$, we have

$$
\int_{\Omega}|\boldsymbol{w}|^{2} d x \leq C \varepsilon^{2} \int_{\Omega}|\boldsymbol{e}(\boldsymbol{w})|^{2} d x+\frac{C}{\varepsilon^{2}} \int_{B_{\varepsilon}}|\boldsymbol{e}(\boldsymbol{w})|^{2} d x, \quad \forall \boldsymbol{w} \in H_{0}^{1}\left(\Omega ; \mathbb{R}^{3}\right) .
$$

If $\kappa>0$, then by (2.9), (6.29) and (6.32) there holds $\int_{\Omega}\left|\boldsymbol{u}_{\varepsilon}(\tau)\right|^{2} d x \leq C \int_{\Omega} \boldsymbol{\sigma}_{\varepsilon}\left(\boldsymbol{u}_{\varepsilon}\right)$ : $\boldsymbol{e}\left(\boldsymbol{u}_{\varepsilon}\right)(\tau) d x \leq C$. Otherwise, if $\kappa=0$, then by (2.10), (6.14) and (6.29) we have

$$
\begin{aligned}
\int_{\Omega}\left|\boldsymbol{u}_{\varepsilon}(\tau)\right|^{2}(\tau) d x & \leq C \int_{\Omega \times(0, T)} \rho_{\varepsilon}\left|\frac{\partial \boldsymbol{u}_{\varepsilon}}{\partial t}\right|^{2} d x d t+C \int_{\Omega} \boldsymbol{\sigma}_{\varepsilon}\left(\boldsymbol{u}_{\varepsilon}\right): \boldsymbol{e}\left(\boldsymbol{u}_{\varepsilon}\right)(\tau) d x+C \int_{\Omega}\left|\boldsymbol{a}_{0}\right|^{2} d x \\
& \leq C .
\end{aligned}
$$

The estimate

$$
\int_{\Omega}\left|\boldsymbol{u}_{\varepsilon}(\tau)\right|^{2} d x \leq C, \quad \forall \tau \in[0, T]
$$

is proved. We deduce from (2.16) and (6.33) that

$$
\int_{\Omega}\left|\boldsymbol{v}_{\varepsilon}\right|^{2}(\tau)+\left|\theta_{\varepsilon}\right|^{2}(\tau) d x \leq C, \quad \forall \tau \in[0, T] .
$$

By substituting $\boldsymbol{u}_{\varepsilon}(\tau)$ for $\boldsymbol{w}$ in (6.31), taking (2.16) and (6.30) into account we infer

$$
\begin{aligned}
\int_{\Omega}\left|v_{\varepsilon 1}(\tau)\right|^{2}+\left|v_{\varepsilon 2}(\tau)\right|^{2}+\left|\frac{v_{\varepsilon 3}}{\varepsilon}(\tau)\right|^{2} d x & \leq \frac{C}{\varepsilon^{2}} \int_{\Omega}\left|\boldsymbol{e}\left(\boldsymbol{u}_{\varepsilon}\right)\right|^{2}(\tau) d x \\
& \leq \frac{C}{\varepsilon^{2} \mu_{1 \varepsilon}}, \quad \forall \tau \in[0, T],
\end{aligned}
$$

which, joined with (6.30), (6.33), (6.34) completes the proof of (6.20). Taking Lemma 6.1 into account, we deduce that the convergences (6.21), (6.26) take place, up to a subsequence, for suitable $\boldsymbol{u}_{0} \in L^{\infty}\left(0, T ; L^{2}\left(\Omega \times Y ; \mathbb{R}^{3}\right)\right),\left(\boldsymbol{\Xi}^{m}, \boldsymbol{\Xi}^{f}\right) \in\left(L^{\infty}\left(0, T ; L^{2}(\Omega \times Y\right.\right.$; $\mathbb{S})))^{2},(\boldsymbol{u}, \boldsymbol{v}) \in\left(L^{\infty}\left(0, T ; L^{2}\left(\Omega ; \mathbb{R}^{3}\right)\right)\right)^{2}, \theta \in L^{\infty}\left(0, T ; L^{2}(\Omega)\right)$. In order to establish the identification relations (6.22), we test the convergences (6.21) with appropriate fields. Choosing first $\boldsymbol{\Psi} \in \mathcal{D}\left(\Omega \times(0, T) ; C_{\sharp}^{\infty}(Y ; \mathbb{S})\right)$ and passing to the limit as $\varepsilon \rightarrow 0$ in the equation

$$
\begin{aligned}
\int_{\Omega \times(0, T)} & \varepsilon \boldsymbol{e}\left(\boldsymbol{u}_{\varepsilon}\right): \boldsymbol{\Psi}\left(x, t, \frac{x}{\varepsilon}\right) d x d t= \\
& -\varepsilon \int_{\Omega \times(0, T)} \boldsymbol{u}_{\varepsilon} \cdot \operatorname{div}_{x} \boldsymbol{\Psi}\left(x, t, \frac{x}{\varepsilon}\right) d x d t-\int_{\Omega \times(0, T)} \boldsymbol{u}_{\varepsilon} \cdot \operatorname{div}_{y} \boldsymbol{\Psi}\left(x, t, \frac{x}{\varepsilon}\right) d x d t,
\end{aligned}
$$

we find $\int_{\Omega \times(0, T) \times Y} \boldsymbol{\Xi}^{m}: \boldsymbol{\Psi} d x d t d y=-\int_{\Omega \times(0, T) \times Y} \boldsymbol{u}_{0} \cdot \boldsymbol{d i v}_{y} \boldsymbol{\Psi} d x d t d y$ and deduce by the arbitrary choice of $\boldsymbol{\Psi}$ that $\boldsymbol{u}_{0} \in L^{\infty}\left(0, T ; L^{2}\left(\Omega ; H_{\sharp}^{1}(Y)\right)\right)$ and $\boldsymbol{e}_{y}\left(\boldsymbol{u}_{0}\right)=\boldsymbol{\Xi}^{m}$. By (6.20), the sequence $\left(\varepsilon \boldsymbol{e}\left(\boldsymbol{u}_{\varepsilon}\right) 1_{B_{\varepsilon}}\right)$ converges strongly to 0 in $L^{2}$. Choosing $\boldsymbol{\Psi} \in$ 
$\mathcal{D}\left(\Omega \times(0, T) ; \mathcal{D}_{\sharp}(B ; \mathbb{S})\right)$ we deduce $\int_{\Omega \times(0, T) \times Y} \boldsymbol{\Xi}^{m}: \boldsymbol{\Psi} d x d t d y=0$. We infer that $\boldsymbol{e}_{y}\left(\boldsymbol{u}_{0}\right)=0$ in $\Omega \times(0, T) \times B$. Therefore, for a. e. $(x, t) \in \Omega \times(0, T)$, the restriction of $\boldsymbol{u}_{0}(x, t,$.$) to B$ is a rigid displacement. By the periodicity of $\boldsymbol{u}_{0}$ there holds $\boldsymbol{u}_{0}\left(x, t, y_{1}, y_{2},-\frac{1}{2}\right)=\boldsymbol{u}_{0}\left(x, t, y_{1}, y_{2}, \frac{1}{2}\right)$, hence

$$
\boldsymbol{u}_{0}=\boldsymbol{a}+b \boldsymbol{e}_{3} \wedge\left(\boldsymbol{y}-\boldsymbol{y}_{B}\right), \quad \text { in } \Omega \times(0, T) \times B,
$$

for a suitable $(\boldsymbol{a}, b) \in L^{\infty}\left(0, T ; L^{2}\left(\Omega ; \mathbb{R}^{3}\right)\right) \times L^{\infty}\left(0, T ; L^{2}(\Omega)\right)$. Fixing $\boldsymbol{\varphi} \in \mathcal{D}(\Omega \times(0, T)$; $\mathbb{R}^{3}$ ), taking (2.2), (2.16), (6.35), the convergences $\boldsymbol{v}_{\varepsilon} \stackrel{*}{\rightarrow} \boldsymbol{v}$ and $\boldsymbol{u}_{\varepsilon} \longrightarrow \boldsymbol{u}_{0}$ and Lemma 6.1 (i) into account, we get

$$
\begin{aligned}
\int_{\Omega \times(0, T)} \boldsymbol{v} \cdot \boldsymbol{\varphi} d x d t & =\frac{1}{|B|} \lim _{\varepsilon \rightarrow 0} \int_{\Omega \times(0, T)} \boldsymbol{u}_{\varepsilon} \cdot \boldsymbol{\varphi}(x, t) 1_{B}\left(\left[\frac{x}{\varepsilon}\right]\right) d x d t \\
& =\frac{1}{|B|} \int_{\Omega \times(0, T) \times B} \boldsymbol{u}_{0} \cdot \boldsymbol{\varphi}(x, t) 1_{B}(y) d x d t d y=\int_{\Omega \times(0, T)} \boldsymbol{a} \cdot \boldsymbol{\varphi} d x d t,
\end{aligned}
$$

hence $\boldsymbol{a}=\boldsymbol{v}=f_{B} \boldsymbol{u}_{0}(., y) d y$. By testing the convergences $\theta_{\varepsilon} \stackrel{*}{\rightarrow} \theta, \boldsymbol{u}_{\varepsilon} \rightarrow \boldsymbol{u}_{0}$ with a function $\varphi \in \mathcal{D}(\Omega \times(0, T))$ and with the sequence $\left(\varphi_{\varepsilon}\right)$ given by $\varphi_{\varepsilon}(x, t):=\varphi(x, t)\left(\boldsymbol{e}_{3} \wedge\right.$ $\left.\left(\left[\frac{\boldsymbol{x}}{\varepsilon}\right]-\boldsymbol{y}_{B}\right)\right) 1_{B}\left(\left[\frac{x}{\varepsilon}\right]\right)\left(\left(\varphi_{\varepsilon}\right)\right.$ is admissible by Lemma 6.1 (i)), thanks to $(2.2),(2.16)$, (6.35) we find

$$
\begin{aligned}
\int_{\Omega \times(0, T)} \theta \varphi d x d t & =\lim _{\varepsilon \rightarrow 0} \int_{\Omega \times(0, T)} \theta_{\varepsilon} \varphi d x d t \\
& =\lim _{\varepsilon \rightarrow 0} \frac{1}{J} \int_{B_{\varepsilon} \times(0, T)} \boldsymbol{u}_{\varepsilon} \cdot\left(\boldsymbol{e}_{3} \wedge\left(\left[\frac{\boldsymbol{x}}{\varepsilon}\right]-\boldsymbol{y}_{B}\right)\right) \varphi d x d t \\
& =\frac{1}{J} \int_{\Omega \times(0, T) \times B} \boldsymbol{u}_{0} \cdot\left(\boldsymbol{e}_{3} \wedge\left(\boldsymbol{y}-\boldsymbol{y}_{B}\right)\right) \varphi d x d t d y \\
& =\frac{1}{J} \int_{\Omega \times(0, T) \times B}\left(\boldsymbol{v}+b \boldsymbol{e}_{3} \wedge\left(\boldsymbol{y}-\boldsymbol{y}_{B}\right)\right) .\left(\boldsymbol{e}_{3} \wedge\left(\boldsymbol{y}-\boldsymbol{y}_{B}\right)\right) \varphi d x d t d y \\
& =\frac{1}{J} \int_{\Omega \times(0, T) \times B} b\left|\boldsymbol{e}_{3} \wedge\left(\boldsymbol{y}-\boldsymbol{y}_{B}\right)\right|^{2} \varphi d x d t d y=\int_{\Omega \times(0, T)} b \varphi d x d t,
\end{aligned}
$$

hence $\theta=b=\frac{1}{J} \int_{B} \boldsymbol{u}_{0}(., y) .\left(\boldsymbol{e}_{3} \wedge\left(\boldsymbol{y}-\boldsymbol{y}_{B}\right)\right) d y$. By (1.1), (2.16) and (6.20), the sequence $\left(v_{\varepsilon 3}\right)$ is bounded in $L^{\infty}\left(0, T ; L^{2}\left(\omega ; H_{0}^{1}(0, L)\right)\right)$, thus $v_{3} \in L^{\infty}\left(0, T ; L^{2}\left(\omega ; H_{0}^{1}(0, L)\right)\right)$. Choosing $\varphi \in \mathcal{D}\left(\Omega \times(0, T) ; C_{\sharp}^{\infty}(Y)\right)$ such that $\frac{\partial \varphi}{\partial y_{3}}=0$ and $\varphi=0$ in $\Omega \times(0, T) \times$ $(Y \backslash B)$ and passing to the limit as $\varepsilon \rightarrow 0$ in the equation $\int_{\Omega \times(0, T)} \frac{\partial u_{\varepsilon}}{\partial x_{3}} \varphi\left(x, t, \frac{x}{\varepsilon}\right) d x=$ $-\int_{\Omega \times(0, T)} u_{\varepsilon 3} \frac{\partial \varphi}{\partial x_{3}}\left(x, t, \frac{x}{\varepsilon}\right) d x d t$, we obtain

$$
\begin{aligned}
\int_{\Omega \times(0, T) \times B} \Xi_{33}^{f} \varphi d x d t d y & =-\int_{\Omega \times(0, T) \times B}\left(\boldsymbol{v}+\theta \boldsymbol{e}_{3} \wedge\left(\boldsymbol{y}-\boldsymbol{y}_{B}\right)\right)_{3} \frac{\partial \varphi}{\partial x_{3}} d x d t d y \\
& =-\int_{\Omega \times(0, T) \times B} v_{3} \frac{\partial \varphi}{\partial x_{3}} d x d t d y .
\end{aligned}
$$

We infer from the arbitrary choice of $\varphi$ that $\int_{-\frac{1}{2}}^{\frac{1}{2}} \Xi_{33}^{f}\left(x, t, y_{1}, y_{2}, s\right) d s=\frac{\partial v_{3}}{\partial x_{3}}$ a.e. in $\Omega \times(0, T) \times D$. The proof of $(6.22)$ is achieved provided we establish that under (2.13), there holds

$$
\begin{aligned}
& \theta \in L^{\infty}\left(0, T ; L^{2}\left(\omega ; H_{0}^{1}(0, L)\right)\right), \\
& \frac{\partial \theta}{\partial x_{3}}=\frac{2}{J} \int_{B}\left(-\left(\boldsymbol{y}-\boldsymbol{y}_{B}\right)_{2} \Xi_{13}^{f}+\left(\boldsymbol{y}-\boldsymbol{y}_{B}\right)_{1} \Xi_{23}^{f}\right) d y .
\end{aligned}
$$


To that aim, we fix $\varphi \in C^{\infty}(\overline{\Omega \times(0, T)})$, set $\varphi=0$ on $\mathbb{R}^{3} \times \overline{(0, T)} \backslash \overline{\Omega \times(0, T)}$ and define

$$
\begin{aligned}
& \bar{\varphi}_{\varepsilon}(x, t):=\sum_{i \in I_{\varepsilon}}\left(f_{D_{\varepsilon}^{i}} \varphi\left(s_{1}, s_{2}, x_{3}, t\right) d s_{1} d s_{2}\right) 1_{P_{\varepsilon}^{i}}\left(x_{1}, x_{2}\right), \\
& P_{\varepsilon}^{i}:=\varepsilon(\{i\}+P), \quad P:=\left(-\frac{1}{2}, \frac{1}{2}\right)^{2}, \\
& D_{\varepsilon}^{i}:=\varepsilon(\{i\}+D), \quad I_{\varepsilon}:=\left\{i \in \mathbb{Z}^{2}, P_{\varepsilon}^{i} \cap \omega \neq \emptyset\right\},
\end{aligned}
$$

$$
\begin{aligned}
& \boldsymbol{M}_{\varepsilon}(x, t):= \\
& \left(\begin{array}{ccc}
0 & 0 & -\left(\left[\frac{x_{2}}{\varepsilon}\right]-\left(\boldsymbol{y}_{B}\right)_{2}\right) \bar{\varphi}_{\varepsilon} \\
0 & 0 & \left(\left[\frac{x_{1}}{\varepsilon}\right]-\left(\boldsymbol{y}_{B}\right)_{1}\right) \bar{\varphi}_{\varepsilon} \\
-\left(\left[\frac{x_{2}}{\varepsilon}\right]-\left(\boldsymbol{y}_{B}\right)_{2}\right) \bar{\varphi}_{\varepsilon} & \left(\left[\frac{x_{1}}{\varepsilon}\right]-\left(\boldsymbol{y}_{B}\right)_{1}\right) \bar{\varphi}_{\varepsilon} & 0
\end{array}\right) 1_{B_{\varepsilon}}(x) .
\end{aligned}
$$

Denoting by $\boldsymbol{n}$ the outward pointing normal to $\partial B_{\varepsilon}$, noticing that $\frac{\partial \bar{\varphi}_{\varepsilon}}{\partial x_{1}}=\frac{\partial \bar{\varphi}_{\varepsilon}}{\partial x_{2}}=0$ in $B_{\varepsilon}$ and that $n_{3}=0$ on $\partial B_{\varepsilon} \cap \Omega$, by integration by parts we get, for all $\tau \in[0, T]$,

$$
\begin{aligned}
& \int_{B_{\varepsilon}} \boldsymbol{e}\left(\boldsymbol{u}_{\varepsilon}\right): \boldsymbol{M}_{\varepsilon}(\tau) d x= \\
& \quad-\int_{B_{\varepsilon}}\left(-u_{\varepsilon 1}\left(\left[\frac{x_{2}}{\varepsilon}\right]-\left(\boldsymbol{y}_{B}\right)_{2}\right)+u_{\varepsilon 2}\left(\left[\frac{x_{1}}{\varepsilon}\right]-\left(\boldsymbol{y}_{B}\right)_{1}\right)\right) \frac{\partial \bar{\varphi}_{\varepsilon}}{\partial x_{3}}(\tau) d x \\
& \quad+\int_{\partial B_{\varepsilon}}\left(-\left(\left[\frac{x_{2}}{\varepsilon}\right]-\left(\boldsymbol{y}_{B}\right)_{2}\right) n_{1}+\left(\left[\frac{x_{1}}{\varepsilon}\right]-\left(\boldsymbol{y}_{B}\right)_{1}\right) n_{2}\right) u_{\varepsilon 3} \bar{\varphi}_{\varepsilon}(\tau) d \mathcal{H}^{2}(x) .
\end{aligned}
$$

If $\liminf _{\varepsilon \rightarrow 0} \varepsilon \mu_{1 \varepsilon}<+\infty$, then under (2.13) the set $D$ is a disk of center 0 , hence by (2.1), (2.7) we have $\boldsymbol{y}_{B}=0, \boldsymbol{n} 1_{\partial B_{\varepsilon} \cap \Omega}=\frac{1}{R}\left(\left[\frac{x_{1}}{\varepsilon}\right] \boldsymbol{e}_{1}+\left[\frac{x_{2}}{\varepsilon}\right] \boldsymbol{e}_{2}\right) 1_{\partial B_{\varepsilon} \cap \Omega}$, therefore the term of the second line of (6.40) is equal to zero. Otherwise, if $\lim _{\varepsilon \rightarrow 0} \varepsilon \mu_{1 \varepsilon}=+\infty$, then by (6.44) the term of the second line of (6.40) is negligible. Taking (2.16) into account, we infer

$$
\int_{B_{\varepsilon}} \boldsymbol{e}\left(\boldsymbol{u}_{\varepsilon}\right): \boldsymbol{M}_{\varepsilon}(\tau) d x=-J \int_{\Omega} \theta_{\varepsilon} \frac{\partial \bar{\varphi}_{\varepsilon}}{\partial x_{3}}(\tau) d x+o(1)
$$

By (6.38), we have

$$
\left.\left\|\varphi-\bar{\varphi}_{\varepsilon}\right\|_{L^{\infty}} \leq C \varepsilon\|\nabla \varphi\|_{L^{\infty}}, \quad\left\|\frac{\partial\left(\varphi-\bar{\varphi}_{\varepsilon}\right)}{\partial x_{3}}\right\|_{L^{\infty}} \leq C \varepsilon\left\|\nabla^{2} \varphi\right\|_{L^{\infty}} \cdot\right)
$$

By (6.42) and (6.3) (applied with $h_{0}=1_{B}, \chi_{0}(x, y):=\varphi(x)\left(\boldsymbol{y}-\boldsymbol{y}_{B}\right)_{\alpha}, \chi_{\varepsilon}(x):=$ $\left.\chi_{0}\left(x,\left[\frac{x}{\varepsilon}\right]\right), \alpha \in\{1,2\}\right)$, there holds

$$
\boldsymbol{M}_{\varepsilon} \longrightarrow\left(\begin{array}{ccc}
0 & 0 & -\left(\boldsymbol{y}-\boldsymbol{y}_{B}\right)_{2} \varphi \\
0 & 0 & \left(\boldsymbol{y}-\boldsymbol{y}_{B}\right)_{1} \varphi \\
-\left(\boldsymbol{y}-\boldsymbol{y}_{B}\right)_{2} \varphi & \left(\boldsymbol{y}-\boldsymbol{y}_{B}\right)_{1} \varphi & 0
\end{array}\right) 1_{B}(y)
$$

By passing to the limit as $\varepsilon \rightarrow 0$ in (6.41), in accordance with (6.2), (6.21), (6.42), we obtain

$$
\begin{aligned}
& 2 \int_{\Omega \times(0, T) \times B}\left(-\left(\boldsymbol{y}-\boldsymbol{y}_{B}\right)_{2} \Xi_{13}^{f}+\left(\boldsymbol{y}-\boldsymbol{y}_{B}\right)_{1} \Xi_{23}^{f}\right) \varphi d x d y d t= \\
&-J \int_{\Omega \times(0, T)} \theta \frac{\partial \varphi}{\partial x_{3}} d x d t .
\end{aligned}
$$


As (6.43) takes place for all $\varphi \in C^{\infty}(\overline{\Omega \times(0, T)})$, we deduce (6.37). The proof of (6.22) is achieved. If $k=+\infty$, we infer from $(2.9),(6.20),(6.21)$ that $v_{3}=0$ and $\boldsymbol{\Xi}^{f}=0$, then from (6.37) that $\theta=0$. If $\kappa=+\infty$, then by (2.9), (6.20), (6.21) we have $\boldsymbol{v}=0$. Assertion (6.23) is proved. If $\kappa>0$, Assertion (6.24) results from (2.9) and (6.20). The relations stated in (6.25) are obtained by fitting the argument developed in [9] (see Proposition 3.8 and the argumentation p.180). If (2.14) is verified, then by (6.20) the sequence $\left(\boldsymbol{u}_{\varepsilon}\right)$ is bounded in $W^{1, \infty}\left(0, T ; L^{2}\left(\Omega ; \mathbb{R}^{3}\right)\right)$. Assertion (6.26) follows then from Lemma 6.1 (ii).

The estimate established in the next lemma is used in the proof of (6.37).

Lemma 6.5. Assume (2.1), (2.7), (2.8). Let $\left(\boldsymbol{u}_{\varepsilon}\right)$ be the sequence of the solutions of (1.1) and let $\boldsymbol{n}$ denote the outward pointing normal to $B_{\varepsilon}$. Let $\varphi \in C(\overline{\Omega \times(0, T)})$ and let $\bar{\varphi}_{\varepsilon}$ be defined by (6.38). Then the following estimate holds

$$
\left|\int_{\partial B_{\varepsilon}}\left(-\left(\left[\frac{x_{2}}{\varepsilon}\right]-\left(\boldsymbol{y}_{B}\right)_{2}\right) n_{1}+\left(\left[\frac{x_{1}}{\varepsilon}\right]-\left(\boldsymbol{y}_{B}\right)_{1}\right) n_{2}\right) u_{\varepsilon 3} \bar{\varphi}_{\varepsilon}(\tau) d \mathcal{H}^{2}\right| \leq \frac{C}{\varepsilon \mu_{1 \varepsilon}} .
$$

Proof. By the inequality (proved below)

$$
\int_{\partial D}\left|w-f_{D} w d \mathcal{L}^{2}\right|^{2} d \mathcal{H}^{1} \leq C \int_{D}|\nabla w|^{2} d y, \forall w \in H^{1}(D),
$$

we have

$$
\int_{\partial D \times] 0, L[}|\boldsymbol{w}-\overline{\boldsymbol{w}}|^{2} d \mathcal{H}^{2} \leq C \int_{D \times(0, L)}|\nabla \boldsymbol{w}|^{2} d x, \forall \boldsymbol{w} \in H^{1}\left(D \times(0, L) ; \mathbb{R}^{3}\right),
$$

where $\overline{\boldsymbol{w}}(x):=f_{D} \boldsymbol{w}\left(s_{1}, s_{2}, x_{3}\right) d s_{1} d s_{2}$. Since $W:=H^{1}\left(D \times(0, L) ; \mathbb{R}^{3}\right) \cap L^{2}\left(D ; H_{0}^{1}(0, L ;\right.$ $\left.\mathbb{R}^{3}\right)$ ) contains no non-vanishing rigid displacement, we infer from Korn's inequality (see [24], Theorem 2.5 p.19) that

$$
\int_{\partial D \times] 0, L[}|\boldsymbol{w}-\overline{\boldsymbol{w}}|^{2} d \mathcal{H}^{2} \leq C \int_{D \times(0, L)}|\boldsymbol{e}(\boldsymbol{w})|^{2} d x, \quad \forall \boldsymbol{w} \in W .
$$

Fixing $\boldsymbol{i}=\left(i_{1}, i_{2}\right) \in \mathbb{Z}^{2}$, setting $w_{\alpha}\left(y_{1}, y_{2}, y_{3}\right):=u_{\varepsilon \alpha}\left(\varepsilon\left(y_{1}-i_{1}\right), \varepsilon\left(y_{2}-i_{2}\right), y_{3}\right), w_{3}(y):=$ $\frac{1}{\varepsilon} u_{\varepsilon 3}\left(\varepsilon\left(y_{1}-i_{1}\right), \varepsilon\left(y_{2}-i_{2}\right), y_{3}\right)$, by making suitable changes of variables in $(6.46)$ and by summation over $i \in I_{\varepsilon}$, where $I_{\varepsilon}$ is defined by (6.38), taking (6.20) into account we deduce

$$
\int_{\partial B_{\varepsilon} \cap \Omega}\left|u_{\varepsilon 3}-\bar{u}_{\varepsilon 3}\right|^{2} d \mathcal{H}^{2} \leq \frac{C}{\varepsilon} \int_{B_{\varepsilon}}\left|\boldsymbol{e}\left(\boldsymbol{u}_{\varepsilon}\right)\right|^{2} d x \leq \frac{C}{\varepsilon \mu_{1 \varepsilon}} .
$$

On the other hand, noticing that by (6.38) there holds $\frac{\partial \bar{g}_{\varepsilon}}{\partial x_{\alpha}}=0$ in $B_{\varepsilon}$ for all $\alpha \in\{1,2\}$ and $g \in H^{1}(\Omega)$, we infer from the Gauss-Green's Theorem that

$$
\int_{\partial B_{\varepsilon}}\left(-\left(\left[\frac{x_{2}}{\varepsilon}\right]-\left(\boldsymbol{y}_{B}\right)_{2}\right) n_{1}+\left(\left[\frac{x_{1}}{\varepsilon}\right]-\left(\boldsymbol{y}_{B}\right)_{1}\right) n_{2}\right) \bar{u}_{\varepsilon 3} \bar{\varphi}_{\varepsilon}(\tau) d \mathcal{H}^{2}(x)=0 .
$$

Assertion (6.44) follows from (6.47) and (6.48).

Proof of (6.45). If (6.45) is false, there exists a sequence $\left(w_{n}\right)$ in $H^{1}(D)$ such that $f_{D} w_{n} d \mathcal{L}^{2}=0, \int_{\partial D}\left|w_{n}\right|^{2} d \mathcal{H}^{1}=1, \lim _{n \rightarrow+\infty} \int_{D}\left|\nabla w_{n}\right|^{2} d y=0$. By the PoincaréWirtinger's inequality $\int_{D}\left|w-f_{D} w d \mathcal{L}^{2}\right|^{2} d y \leq C \int_{D}|\nabla w|^{2} d y$, there holds $w_{n} \rightarrow 0$ in 
$H^{1}(D)$, hence, by the continuity of the trace application from $H^{1}(D)$ to $L^{2}(\partial D)$, $\int_{\partial D}\left|w_{n}\right|^{2} d \mathcal{H}^{1} \rightarrow 0$. This contradiction establishes (6.45).

Justification of Remark $2.2(v)$. Assume that $\rho_{\varepsilon}>>1$ on some $\varepsilon$-periodic subset $G_{\varepsilon}$ of $\Omega$ (that is $1_{G_{\varepsilon}}=1_{G}\left(\frac{x}{\varepsilon}\right)$ for some $\left.G \subset Y\right)$. Then by (6.20) the sequence $\left(\frac{\partial \boldsymbol{u}_{\varepsilon}}{\partial t} 1_{G_{\varepsilon}}\right)$ two-scale converges to 0 . Noticing that by $(6.3)$ there holds $\left(\boldsymbol{u}_{\varepsilon} 1_{G_{\varepsilon}}\right) \rightarrow \boldsymbol{u}_{0} 1_{G}$ and $\left(\boldsymbol{u}_{\varepsilon}(0) 1_{G_{\varepsilon}}\right) \longrightarrow \boldsymbol{a}_{0} 1_{G}$, we deduce from Lemma 6.1 (ii) that $\frac{\partial \boldsymbol{u}_{0}}{\partial t} 1_{G}=0$, hence

$$
\boldsymbol{u}_{0}(\tau) 1_{G}=\boldsymbol{u}_{0}(0) 1_{G}=a_{0} 1_{G}, \forall \tau \in[0, T]
$$

7. Proof of Theorem 2.1. Our proof, which combines the energy method of Tartar [33] with the two-scale convergence method of Allaire and Nguetseng [2], [23], relies on the appropriate choice of an admissible sequence of oscillating test fields $\left(\phi_{\varepsilon}\right)$. We will multiply (1.1) by $\left(\phi_{\varepsilon}\right)$ and, by passing to the limit as $\varepsilon \rightarrow 0$ in accordance with the convergences (6.21) established in Proposition 6.4, we will obtain the variational problem satisfied by the triple $\left(\boldsymbol{u}_{0}, \boldsymbol{v}, \theta\right)$ given, according to the order of magnitude of $k$ and $\kappa$, by (7.21), (7.38) or (7.44). Then, noticing that this variational problem is equivalent to (6.13) for a suitable choice of $H, V, a, h, \xi_{0}, \xi_{1}$, we will deduce from Theorem 6.2 the existence, the uniqueness and the regularity of its solution and the initial-boundary conditions. Consequently, the convergences established in (6.21) for subsequences of $\left(\boldsymbol{u}_{\varepsilon}\right),\left(\boldsymbol{v}_{\varepsilon}\right),\left(\theta_{\varepsilon}\right)$, take place for the complete sequences. Then, we will prove that this variational problem is equivalent to (2.17). Finally, we will establish the corrector result $(2.25)$. We set

$$
\begin{aligned}
& H:=\left\{\left(\boldsymbol{w}_{0}, \boldsymbol{\psi}, \varphi\right) \in L^{2}\left(\Omega \times Y ; \mathbb{R}^{3}\right) \times L^{2}\left(\Omega ; \mathbb{R}^{3}\right) \times L^{2}(\Omega),\right. \\
& \left.\boldsymbol{w}_{0}=\boldsymbol{\psi}+\varphi \boldsymbol{e}_{3} \wedge\left(\boldsymbol{y}-\boldsymbol{y}_{B}\right) \text { in } \Omega \times B\right\}, \\
& \left(\left(\boldsymbol{w}_{0}, \boldsymbol{\psi}, \varphi\right),\left(\tilde{\boldsymbol{w}}_{0}, \tilde{\boldsymbol{\psi}}, \tilde{\varphi}\right)\right)_{H}:=\int_{\Omega \times Y} \rho \boldsymbol{w}_{0} . \tilde{\boldsymbol{w}}_{0} d x d y .
\end{aligned}
$$

By (2.14), (2.15) there holds $0<c \leq \rho \leq C<+\infty$, hence the application $(.,)_{H}$ is an inner product on $H$ and the associated norm is equivalent to $\left(\int_{\Omega \times Y}\left|\boldsymbol{w}_{0}\right|^{2} d x d y\right)^{\frac{1}{2}}=$ $\left(\int_{\Omega \times(Y \backslash B)}\left|\boldsymbol{w}_{0}\right|^{2} d x d y+|B| \int_{\Omega}|\boldsymbol{\psi}|^{2} d x+J \int_{\Omega}|\varphi|^{2} d x\right)^{\frac{1}{2}}$ (see $\left.(2.2)\right)$. If $\left(\left(\boldsymbol{w}_{0 n}, \boldsymbol{\psi}_{n}, \varphi_{n}\right)\right)$ is a Cauchy sequence in $H$, then the sequences $\left(\boldsymbol{w}_{0 n}\right),\left(\boldsymbol{\psi}_{n}\right),\left(\varphi_{n}\right)$ converge strongly in $L^{2}$ and, up to a subsequence, almost everywhere respectively to some $\boldsymbol{w}_{0}, \boldsymbol{\psi}, \varphi$. Since $\boldsymbol{w}_{0 n}=\boldsymbol{\psi}_{n}+\varphi_{n} \boldsymbol{e}_{3} \wedge\left(\boldsymbol{y}-\boldsymbol{y}_{B}\right)$ in $\Omega \times B, \forall n \in \mathbb{N}$, there holds $\boldsymbol{w}_{0}=\boldsymbol{\psi}+\varphi \boldsymbol{e}_{3} \wedge\left(\boldsymbol{y}-\boldsymbol{y}_{B}\right)$ in $\Omega \times B$, thus $\left(\boldsymbol{w}_{0}, \boldsymbol{\psi}, \varphi\right) \in H$. We infer that $H$ is a Hilbert space. In order to define $\left(\phi_{\varepsilon}\right)$, we choose $\left(\boldsymbol{w}_{0}, \boldsymbol{\psi}, \varphi\right) \in L^{2}(0, T ; H)$ satisfying

$$
\begin{gathered}
\boldsymbol{w}_{0} \in C^{\infty}\left([0, T] ; \mathcal{D}\left(\Omega ; C_{\sharp}^{\infty}\left(Y ; \mathbb{R}^{3}\right)\right)\right), \\
\boldsymbol{w}_{0}(T)=\frac{\partial \boldsymbol{w}_{0}}{\partial t}(T)=0,
\end{gathered}
$$

set

$$
B^{\varepsilon}:=\{\boldsymbol{y} \in Y, \operatorname{dist}(\boldsymbol{y}, B)<\varepsilon\}, \quad B_{\sharp}^{\varepsilon}:=\bigcup_{i \in \mathbb{Z}^{3}}\{i\}+B^{\varepsilon}, \quad B_{\varepsilon}^{\varepsilon}=\Omega \cap \varepsilon B_{\sharp}^{\varepsilon},
$$


( $B_{\varepsilon}^{\varepsilon}$ denotes the $\varepsilon^{2}$-neighborhood of $B_{\varepsilon}$ in $\Omega$ ), fix $\eta_{\varepsilon} \in C_{\sharp}^{\infty}(Y)$ such that

$$
0 \leq \eta_{\varepsilon} \leq 1, \quad \eta_{\varepsilon}=1 \quad \text { in } \quad B, \quad \eta_{\varepsilon}=0 \quad \text { in } \quad Y \backslash B^{\varepsilon}, \quad\left|\nabla \eta_{\varepsilon}\right|<\frac{C}{\varepsilon}
$$

and introduce the field $\chi_{\varepsilon}$ given, according to the order of magnitude of $k$ and $\kappa$ by (7.17), (7.42) or (7.46). Notice that there holds

$$
\eta_{\varepsilon}\left(\frac{x}{\varepsilon}\right)\left(\left|\chi_{\varepsilon}\right|+\left|\frac{\partial \chi_{\varepsilon}}{\partial t}\right|+\left|\frac{\partial^{2} \chi_{\varepsilon}}{\partial t^{2}}\right|\right)\left(x, t, \frac{x}{\varepsilon}\right) \leq C \varepsilon, \quad\left|\boldsymbol{e}\left(\boldsymbol{\chi}_{\varepsilon}\left(x, t, \frac{x}{\varepsilon}\right)\right)\right| \leq C
$$

and that, due to $(7.2)$, we have $\boldsymbol{\chi}_{\varepsilon}=0$ on $\left.\left.\partial \Omega \times\right] 0, T\right]$ for small epsilons. Then we set

$$
\phi_{\varepsilon}(x, t):=\eta_{\varepsilon}\left(\frac{x}{\varepsilon}\right) \boldsymbol{\chi}_{\varepsilon}\left(x, t, \frac{x}{\varepsilon}\right)+\boldsymbol{w}_{0}\left(x, t, \frac{x}{\varepsilon}\right) .
$$

By multiplying (1.1) by $\phi_{\varepsilon}$, after integrations by parts we obtain

$$
\begin{aligned}
\int_{\Omega \times(0, T)} \rho_{\varepsilon} \boldsymbol{u}_{\varepsilon} \cdot \frac{\partial^{2} \phi_{\varepsilon}}{\partial t^{2}} & d x d t+\int_{\Omega} \rho_{\varepsilon} \boldsymbol{a}_{0} \cdot \frac{\partial \phi_{\varepsilon}}{\partial t}(0) d x-\int_{\Omega} \rho_{\varepsilon} \boldsymbol{b}_{0} \cdot \boldsymbol{\phi}_{\varepsilon}(0) d x \\
& +\int_{\Omega \times(0, T)} \boldsymbol{e}\left(\boldsymbol{u}_{\varepsilon}\right): \boldsymbol{\sigma}_{\varepsilon}\left(\boldsymbol{\phi}_{\varepsilon}\right) d x d t=\int_{\Omega \times(0, T)} \rho_{\varepsilon} \boldsymbol{f} . \boldsymbol{\phi}_{\varepsilon} d x d t .
\end{aligned}
$$

By (7.6) and (7.7) there holds

$$
\left|\boldsymbol{\phi}_{\varepsilon}-\boldsymbol{w}_{0}\left(x, t, \frac{x}{\varepsilon}\right)\right|+\left|\frac{\partial^{n} \boldsymbol{\phi}_{\varepsilon}}{\partial t^{n}}-\frac{\partial^{n} \boldsymbol{w}_{0}}{\partial t^{n}}\left(x, t, \frac{x}{\varepsilon}\right)\right| \leq C \varepsilon, \quad(n \in\{1,2\}) .
$$

We deduce from (2.11), (7.9), (6.3) (applied to $\chi_{\varepsilon}=\rho_{\varepsilon}, h_{0} \in\left\{\boldsymbol{w}_{0}, \frac{\partial^{2} \boldsymbol{w}_{0}}{\partial t^{2}}, \boldsymbol{w}_{0}(0)\right.$, $\left.\left.\frac{\partial^{2} \boldsymbol{w}_{0}}{\partial t^{2}}(0)\right\}\right)$ that

$$
\begin{aligned}
\rho_{\varepsilon} \boldsymbol{\phi}_{\varepsilon} \longrightarrow \rho \boldsymbol{w}_{0}, & \rho_{\varepsilon} \frac{\partial^{n} \boldsymbol{\phi}_{\varepsilon}}{\partial t^{n}} \longrightarrow \rho \frac{\partial^{n} \boldsymbol{w}_{0}}{\partial t^{n}}, \quad(n \in\{1,2\}), \\
\rho_{\varepsilon} \boldsymbol{\phi}_{\varepsilon}(0) \longrightarrow \rho \boldsymbol{w}_{0}(0), & \rho_{\varepsilon} \frac{\partial \boldsymbol{\phi}_{\varepsilon}}{\partial t}(0) \longrightarrow \rho \frac{\partial \boldsymbol{w}_{0}}{\partial t}(0) .
\end{aligned}
$$

Since by (6.2), (6.21), (7.10), we have

$$
\begin{aligned}
& \lim _{\varepsilon \rightarrow 0} \int_{\Omega \times(0, T)} \rho_{\varepsilon} \boldsymbol{u}_{\varepsilon} \cdot \frac{\partial^{2} \boldsymbol{\phi}_{\varepsilon}}{\partial t^{2}} d x d t+\int_{\Omega} \rho_{\varepsilon} \boldsymbol{a}_{0} \cdot \frac{\partial \phi_{\varepsilon}}{\partial t}(0) d x-\int_{\Omega} \rho_{\varepsilon} \boldsymbol{b}_{0} \cdot \boldsymbol{\phi}_{\varepsilon}(0) d x= \\
& \int_{\Omega \times(0, T) \times Y} \rho \boldsymbol{u}_{0} \cdot \frac{\partial^{2} \boldsymbol{w}_{0}}{\partial t^{2}} d x d t d y+\int_{\Omega \times Y} \rho \boldsymbol{a}_{0} \cdot \frac{\partial \boldsymbol{w}_{0}}{\partial t}(0) d x d y-\int_{\Omega \times Y} \rho \boldsymbol{b}_{0} \cdot \boldsymbol{w}_{0}(0) d x d y, \\
& \lim _{\varepsilon \rightarrow 0} \int_{\Omega \times(0, T)} \rho_{\varepsilon} \boldsymbol{f} \cdot \boldsymbol{\phi}_{\varepsilon} d x d t=\int_{\Omega \times(0, T) \times Y} \rho \boldsymbol{f} \cdot \boldsymbol{w}_{0} d x d t d y,
\end{aligned}
$$

we only have to evaluate the limit of $\left(\int_{\Omega \times(0, T)} \boldsymbol{e}\left(\boldsymbol{u}_{\varepsilon}\right): \boldsymbol{\sigma}_{\varepsilon}\left(\phi_{\varepsilon}\right) d x d t\right)$. To that aim, we 
set

$$
\begin{aligned}
& \int_{\Omega \times(0, T)} \boldsymbol{e}\left(\boldsymbol{u}_{\varepsilon}\right): \boldsymbol{\sigma}_{\varepsilon}\left(\phi_{\varepsilon}\right) d x d t=I_{1 \varepsilon}+I_{2 \varepsilon}+I_{3 \varepsilon} ; \\
& I_{1 \varepsilon}:=\int_{\Omega \backslash B_{\varepsilon}^{\varepsilon} \times(0, T)} \boldsymbol{e}\left(\boldsymbol{u}_{\varepsilon}\right): \boldsymbol{\sigma}_{\varepsilon}\left(\phi_{\varepsilon}\right) d x d t, \\
& I_{2 \varepsilon}:=\int_{B_{\varepsilon} \backslash B_{\varepsilon} \times(0, T)} \boldsymbol{e}\left(\boldsymbol{u}_{\varepsilon}\right): \boldsymbol{\sigma}_{\varepsilon}\left(\phi_{\varepsilon}\right) d x d t, \\
& I_{3 \varepsilon}:=\int_{B_{\varepsilon} \times(0, T)} e\left(\boldsymbol{u}_{\varepsilon}\right): \boldsymbol{\sigma}_{\varepsilon}\left(\phi_{\varepsilon}\right) d x d t .
\end{aligned}
$$

By (7.5), (7.7) there holds $\boldsymbol{\phi}_{\varepsilon} 1_{\Omega \backslash B_{\varepsilon}^{\varepsilon}}=\boldsymbol{w}_{0}\left(x, t, \frac{x}{\varepsilon}\right) 1_{\Omega \backslash B_{\varepsilon}^{\varepsilon}},\left|\frac{1}{\varepsilon} \boldsymbol{\sigma}_{\varepsilon}\left(\boldsymbol{\phi}_{\varepsilon}\right)-\boldsymbol{\sigma}_{0 y}\left(\boldsymbol{w}_{0}\right)\left(x, t, \frac{x}{\varepsilon}\right)\right|$ $1_{\Omega \backslash B_{\varepsilon}^{\varepsilon}} \leq C \varepsilon$, hence by (6.3) applied with $h_{0}:=\boldsymbol{\sigma}_{0 y}\left(\boldsymbol{w}_{0}\right)$ and $\chi_{\varepsilon}=1_{\Omega \backslash B_{\varepsilon}^{\varepsilon}}$, we have

$$
\frac{1}{\varepsilon} \boldsymbol{\sigma}_{\varepsilon}\left(\phi_{\varepsilon}\right) 1_{\Omega \backslash B_{\varepsilon}^{\varepsilon}} \longrightarrow \boldsymbol{\sigma}_{0 y}\left(\boldsymbol{w}_{0}\right) 1_{Y \backslash B}(y),
$$

yielding, thanks to the convergence $\varepsilon \boldsymbol{e}\left(\boldsymbol{u}_{\varepsilon}\right) \longrightarrow \boldsymbol{e}_{y}\left(\boldsymbol{u}_{0}\right)$ (see (6.21), (6.22)),

$$
\lim _{\varepsilon \rightarrow 0} I_{1 \varepsilon}=\int_{\Omega \times(0, T) \times Y \backslash B} \boldsymbol{e}_{y}\left(\boldsymbol{u}_{0}\right): \boldsymbol{\sigma}_{0 y}\left(\boldsymbol{w}_{0}\right) d x d t d y .
$$

By (7.4), (7.5), (7.6), (7.7) there holds $\left|\frac{1}{\varepsilon} \boldsymbol{\sigma}_{\varepsilon}\left(\phi_{\varepsilon}\right) 1_{B_{\varepsilon}^{\varepsilon} \backslash B_{\varepsilon}}\right| \leq C$ and $\mathcal{L}^{3}\left(B_{\varepsilon}^{\varepsilon} \backslash B_{\varepsilon}\right) \leq C \varepsilon$, therefore

$$
\frac{1}{\varepsilon} \sigma_{\varepsilon}\left(\phi_{\varepsilon}\right) 1_{B_{\varepsilon}^{\varepsilon} \backslash B_{\varepsilon}} \longrightarrow 0
$$

and

$$
\lim _{\varepsilon \rightarrow 0} I_{2 \varepsilon}=0 .
$$

We distinguish then several cases:

Case $0<k<+\infty$. We set

$$
\begin{aligned}
& \boldsymbol{\chi}_{\varepsilon}(x, t, y):= \\
& \overline{\boldsymbol{\psi}}_{\varepsilon}(x, t)+\bar{\varphi}_{\varepsilon}(x, t) \boldsymbol{e}_{3} \wedge\left(\boldsymbol{y}-\boldsymbol{y}_{B}\right)-\boldsymbol{w}_{0}(x, t, y)+\varepsilon \boldsymbol{w}_{1 \varepsilon}(x, t, y),
\end{aligned}
$$

where $\boldsymbol{y}_{B}$ is given by $(2.2), \overline{\boldsymbol{\psi}}_{\varepsilon}, \bar{\varphi}_{\varepsilon}$ by $(6.38)$ and $\boldsymbol{w}_{1 \varepsilon} \in L_{\sharp}^{2}\left(Y ; L^{2}\left(\Omega \times(0, T) ; \mathbb{R}^{3}\right)\right)$ by

$$
\boldsymbol{w}_{1 \varepsilon}(x, t, y):=\left(\begin{array}{c}
-\frac{l_{\varepsilon}}{2\left(l_{\varepsilon}+1\right)} \frac{\partial \bar{\psi}_{\varepsilon 3}}{\partial x_{3}}\left(\boldsymbol{y}-\boldsymbol{y}_{B}\right)_{1} \\
-\frac{l_{\varepsilon}}{2\left(l_{\varepsilon}+1\right)} \frac{\partial \bar{\psi}_{\varepsilon 3}}{\partial x_{3}}\left(\boldsymbol{y}-\boldsymbol{y}_{B}\right)_{2} \\
-\frac{\partial \bar{\psi}_{\varepsilon 1}}{\partial x_{3}}\left(\boldsymbol{y}-\boldsymbol{y}_{B}\right)_{1}-\frac{\partial \bar{\psi}_{\varepsilon 2}}{\partial x_{3}}\left(\boldsymbol{y}-\boldsymbol{y}_{B}\right)_{2}
\end{array}\right), \quad \forall y \in Y .
$$

By (7.7) and (7.17) we have $\boldsymbol{\phi}_{\varepsilon}=\overline{\boldsymbol{\psi}}_{\varepsilon}(x, t)+\bar{\varphi}_{\varepsilon}(x, t) \boldsymbol{e}_{3} \wedge\left(\left[\frac{\boldsymbol{x}}{\varepsilon}\right]-\boldsymbol{y}_{B}\right)+\varepsilon \boldsymbol{w}_{1 \varepsilon}\left(x, t,\left[\frac{\boldsymbol{x}}{\varepsilon}\right]\right)$ in $B_{\varepsilon}$, hence

$$
\begin{aligned}
& \boldsymbol{\sigma}_{\varepsilon}\left(\boldsymbol{\phi}_{\varepsilon}\right) 1_{B_{\varepsilon}}= \\
& \mu_{1 \varepsilon}\left(\begin{array}{ccc}
0 & 0 & -\frac{\partial \bar{\varphi}_{\varepsilon}}{\partial x_{3}}\left(\left[\frac{x_{2}}{\varepsilon}\right]-\left(\boldsymbol{y}_{B}\right)_{2}\right) \\
0 & 0 & \frac{\partial \underline{\varphi}_{\varepsilon}}{\partial x_{3}}\left(\left[\frac{x_{1}}{\varepsilon}\right]-\left(\boldsymbol{y}_{B}\right)_{1}\right) \\
-\frac{\partial \overline{\boldsymbol{\varphi}}_{\varepsilon}}{\partial x_{3}}\left(\left[\frac{x_{2}}{\varepsilon}\right]-\left(\boldsymbol{y}_{B}\right)_{2}\right) & \frac{\partial \bar{\varphi}_{\varepsilon}}{\partial x_{3}}\left(\left[\frac{x_{1}}{\varepsilon}\right]-\left(\boldsymbol{y}_{B}\right)_{1}\right) & \frac{3 l_{\varepsilon}+2}{l_{\varepsilon}+1} \frac{\partial \bar{\psi}_{\varepsilon 3}}{\partial x_{3}}
\end{array}\right) 1_{B_{\varepsilon}} \\
& +\varepsilon\left(\lambda_{1 \varepsilon} \operatorname{tr} \boldsymbol{e}_{x}\left(\boldsymbol{w}_{1 \varepsilon}\right)\left(x, t, \frac{x}{\varepsilon}\right) \boldsymbol{I}+2 \mu_{1 \varepsilon} \boldsymbol{e}_{x}\left(\boldsymbol{w}_{1 \varepsilon}\right)\left(x, t, \frac{x}{\varepsilon}\right)\right) 1_{B_{\varepsilon}} .
\end{aligned}
$$


We deduce from (2.8), (2.9), (6.3), (6.42) that

$$
\begin{aligned}
& \boldsymbol{\sigma}_{\varepsilon}\left(\phi_{\varepsilon}\right) 1_{B_{\varepsilon}} \longrightarrow \\
& \quad k\left(\begin{array}{ccc}
0 & 0 & -\frac{\partial \varphi}{\partial x_{3}}\left(\boldsymbol{y}-\boldsymbol{y}_{B}\right)_{2} \\
0 & 0 & \frac{\partial \varphi}{\partial x_{3}}\left(\boldsymbol{y}-\boldsymbol{y}_{B}\right)_{1} \\
-\frac{\partial \varphi}{\partial x_{3}}\left(\boldsymbol{y}-\boldsymbol{y}_{B}\right)_{2} & \frac{\partial \varphi}{\partial x_{3}}\left(\boldsymbol{y}-\boldsymbol{y}_{B}\right)_{1} & \frac{3 l+2}{l+1} \frac{\partial \psi_{3}}{\partial x_{3}}
\end{array}\right) 1_{B}(y),
\end{aligned}
$$

and then, taking the convergence $\boldsymbol{e}\left(\boldsymbol{u}_{\varepsilon}\right) 1_{B_{\varepsilon}} \longrightarrow \boldsymbol{\Xi}^{f}$ (see (6.21)) and (6.22) into account, infer

$$
\begin{aligned}
& \lim _{\varepsilon \rightarrow 0} I_{3 \varepsilon} \\
& =k \int_{\Omega \times(0, T) \times B}\left(\Xi_{33}^{f} \frac{3 l+2}{l+1} \frac{\partial \psi_{3}}{\partial x_{3}}-2 \Xi_{13}^{f} \frac{\partial \varphi}{\partial x_{3}}\left(\boldsymbol{y}-\boldsymbol{y}_{B}\right)_{2}+2 \Xi_{23}^{f} \frac{\partial \varphi}{\partial x_{3}}\left(\boldsymbol{y}-\boldsymbol{y}_{B}\right)_{1}\right) d x d t d y \\
& \quad=k|B| \frac{3 l+2}{l+1} \int_{\Omega \times(0, T)} \frac{\partial v_{3}}{\partial x_{3}} \frac{\partial \psi_{3}}{\partial x_{3}} d x d t+k J \int_{\Omega \times(0, T)} \frac{\partial \theta}{\partial x_{3}} \frac{\partial \varphi}{\partial x_{3}} d x d t .
\end{aligned}
$$

Passing to the limit as $\varepsilon \rightarrow 0$ in (7.8), thanks to (7.11), (7.12), (7.14), (7.16), (7.20), we obtain

$$
\begin{aligned}
\int_{\Omega \times(0, T) \times Y} \rho \boldsymbol{u}_{0} \cdot \frac{\partial^{2} \boldsymbol{w}_{0}}{\partial t^{2}} d x d t d y+\int_{\Omega \times Y} \rho \boldsymbol{a}_{0} \cdot \frac{\partial \boldsymbol{w}_{0}}{\partial t}(0) d x d y \\
-\int_{\Omega \times Y} \rho \boldsymbol{b}_{0} \cdot \boldsymbol{w}_{0}(0) d x d y+\int_{\Omega \times(0, T) \times(Y \backslash B)} \boldsymbol{e}_{y}\left(\boldsymbol{u}_{0}\right): \boldsymbol{\sigma}_{0 y}\left(\boldsymbol{w}_{0}\right) d x d t d y \\
+k|B| \frac{3 l+2}{l+1} \int_{\Omega \times(0, T)} \frac{\partial v_{3}}{\partial x_{3}} \frac{\partial \psi_{3}}{\partial x_{3}} d x d t+k J \int_{\Omega \times(0, T)} \frac{\partial \theta}{\partial x_{3}} \frac{\partial \varphi}{\partial x_{3}} d x d t \\
=\int_{\Omega \times(0, T) \times Y} \rho \boldsymbol{f} \cdot \boldsymbol{w}_{0} d x d t d y,
\end{aligned}
$$

for all $\left(\boldsymbol{w}_{0}, \boldsymbol{\psi}, \varphi\right) \in L^{2}(0, T ; H)$ satisfying $(7.2),(7.3)$. We set

$$
\begin{aligned}
& \xi=\left(\boldsymbol{u}_{0}, \boldsymbol{v}, \theta\right), \quad \xi_{0}=\left(\boldsymbol{a}_{0}, \boldsymbol{a}_{0}, 0\right), \quad \xi_{1}=\left(\boldsymbol{b}_{0}, \boldsymbol{b}_{0}, 0\right), \quad h=(\boldsymbol{f}, \boldsymbol{f}, 0), \\
& V:=\left\{\left(\boldsymbol{w}_{0}, \boldsymbol{\psi}, \varphi\right) \in H, \psi_{3}, \varphi \in L^{2}\left(\omega ; H_{0}^{1}(0, L)\right), \boldsymbol{w}_{0} \in L^{2}\left(\Omega ; H_{\sharp}^{1}\left(Y ; \mathbb{R}^{3}\right)\right)\right\}, \\
& \bar{a}((\boldsymbol{v}, \theta),(\boldsymbol{\psi}, \varphi)):=k|B| \frac{3 l+2}{l+1} \int_{\Omega} \frac{\partial v_{3}}{\partial x_{3}} \frac{\partial \psi_{3}}{\partial x_{3}} d x+k J \int_{\Omega} \frac{\partial \theta}{\partial x_{3}} \frac{\partial \varphi}{\partial x_{3}} d x, \\
& a\left(\left(\boldsymbol{u}_{0}, \boldsymbol{v}, \theta\right),\left(\boldsymbol{w}_{0}, \boldsymbol{\psi}, \varphi\right)\right):=\int_{\Omega \times(Y \backslash B)} \boldsymbol{e}_{y}\left(\boldsymbol{u}_{0}\right): \boldsymbol{\sigma}_{y}\left(\boldsymbol{w}_{0}\right) d x d y+\bar{a}((\boldsymbol{v}, \theta),(\boldsymbol{\psi}, \varphi)), \\
& \left(\left(\left(\boldsymbol{u}_{0}, \boldsymbol{v}, \theta\right),\left(\boldsymbol{w}_{0}, \boldsymbol{\psi}, \varphi\right)\right)\right)_{V}:=\left(\left(\boldsymbol{u}_{0}, \boldsymbol{v}, \theta\right),\left(\boldsymbol{w}_{0}, \boldsymbol{\psi}, \varphi\right)\right)_{H}+\bar{a}((\boldsymbol{v}, \theta),(\boldsymbol{\psi}, \varphi)) \\
& +\int_{\Omega \times(Y \backslash B)} \boldsymbol{\nabla}_{y} \boldsymbol{u}_{0} . \boldsymbol{\nabla}_{y} \boldsymbol{w}_{0} d x d y .
\end{aligned}
$$

By (6.22) and (6.26) there holds $\xi \in L^{2}(0, T ; V), \frac{\partial \xi}{\partial t} \in L^{2}(0, T ; H)$, hence by a density argument the variational formulation (7.21) is equivalent to (6.13). By (7.1), (7.22) and the second Korn's inequality in $H^{1}\left(\Omega \times(Y \backslash B) ; \mathbb{R}^{3}\right)$ (see [24], p. 14), for all $\tilde{\xi}=\left(\boldsymbol{w}_{0}, \boldsymbol{\psi}, \varphi\right) \in V$ we have

$$
\begin{aligned}
\|\tilde{\xi}\|_{V}^{2} & \leq C\left\|\boldsymbol{w}_{0}\right\|_{H^{1}\left(\Omega \times(Y \backslash B) ; \mathbb{R}^{3}\right)}^{2}+C a(\tilde{\xi}, \tilde{\xi}) \\
& \leq C\left\|\boldsymbol{w}_{0}\right\|_{L^{2}\left(\Omega \times(Y \backslash B) ; \mathbb{R}^{3}\right)}^{2}+C\left\|\boldsymbol{e}\left(\boldsymbol{w}_{0}\right)\right\|_{L^{2}\left(\Omega \times(Y \backslash B) ; \mathbb{R}^{3}\right)}^{2}+C a(\tilde{\xi}, \tilde{\xi}) \\
& \leq C|\tilde{\xi}|_{H}^{2}+C a(\tilde{\xi}, \tilde{\xi}),
\end{aligned}
$$


yielding (6.9). Applying Theorem 6.2, we deduce that $\xi=\left(\boldsymbol{u}_{0}, \boldsymbol{v}, \theta\right)$ is the unique solution of (7.21) and, taking (6.10), (6.11), (7.22) into account, that

$$
\xi \in C([0, T] ; V) \cap C^{1}([0, T] ; H), \xi(0)=\left(\boldsymbol{a}_{0}, \boldsymbol{a}_{0}, 0\right), \frac{\partial \xi}{\partial t}(0)=\left(\boldsymbol{b}_{0}, \boldsymbol{b}_{0}, 0\right) .
$$

We infer from (7.24), from the following inequalities (deduced from (7.1), (7.22))

$$
\begin{aligned}
&\left\|\boldsymbol{w}_{0}\right\|_{L^{2}\left(\Omega ; H_{\sharp}^{1}\left(Y ; \mathbb{R}^{3}\right)\right)}+\|\boldsymbol{\psi}\|_{L^{2}\left(\Omega ; \mathbb{R}^{3}\right)}+\left\|\psi_{3}\right\|_{L^{2}\left(\omega ; H_{0}^{1}(0, L)\right)} \\
&+\|\varphi\|_{L^{2}\left(\omega ; H_{0}^{1}(0, L)\right)} \leq C\left\|\left(\boldsymbol{w}_{0}, \boldsymbol{\psi}, \varphi\right)\right\|_{V}, \quad \forall\left(\boldsymbol{w}_{0}, \boldsymbol{\psi}, \varphi\right) \in V, \\
&\left\|\boldsymbol{w}_{0}\right\|_{L^{2}\left(\Omega \times Y ; \mathbb{R}^{3}\right)}+\|\boldsymbol{\psi}\|_{L^{2}\left(\Omega ; \mathbb{R}^{3}\right)}+\|\varphi\|_{L^{2}(\Omega)} \leq C\left|\left(\boldsymbol{w}_{0}, \boldsymbol{\psi}, \varphi\right)\right|_{H}, \\
& \quad \forall\left(\boldsymbol{w}_{0}, \boldsymbol{\psi}, \varphi\right) \in H,
\end{aligned}
$$

and from the next elementary implication (verified by any pair of normed linear spaces $\left(E_{1}, E_{2}\right)$

$$
\begin{aligned}
& \Upsilon \in C^{k}\left([0, T] ; E_{1}\right) \text { and } L \in \mathcal{L}\left(E_{1}, E_{2}\right) \Rightarrow \\
& {\left[L \circ \Upsilon \in C^{k}\left([0, T] ; E_{2}\right) \text { and } \frac{d^{s}}{d t^{s}}(L \circ \Upsilon)=L \circ \frac{d^{s}}{d t^{s}} \Upsilon(s \leq k)\right],}
\end{aligned}
$$

applied to $\Upsilon=\xi=\left(\boldsymbol{u}_{0}, \boldsymbol{v}, \theta\right), E_{1} \in\{H, V\}, E_{2} \in\left\{L^{2}\left(\Omega ; H_{\sharp}^{1}\left(Y ; \mathbb{R}^{3}\right)\right), L^{2}\left(\Omega ; \mathbb{R}^{3}\right), L^{2}(\omega ;\right.$ $\left.\left.H_{0}^{1}(0, L)\right), L^{2}\left(\Omega \times Y ; \mathbb{R}^{3}\right), L^{2}(\Omega)\right\}$ and $L$ chosen among the seven continuous linear operators characterized by $(7.25)$, that

$$
\begin{aligned}
& \boldsymbol{u}_{0} \in C\left([0, T] ; L^{2}\left(\Omega ; H_{\sharp}^{1}\left(Y ; \mathbb{R}^{3}\right)\right)\right) \cap C^{1}\left([0, T] ; L^{2}\left(\Omega \times Y ; \mathbb{R}^{3}\right)\right), \\
& \boldsymbol{u}_{0}(0)=\boldsymbol{a}_{0}, \frac{\partial \boldsymbol{u}_{0}}{\partial t}(0)=\boldsymbol{b}_{0}, \\
& \boldsymbol{v} \in C^{1}\left([0, T] ; L^{2}\left(\Omega ; \mathbb{R}^{3}\right)\right), \boldsymbol{v}(0)=\boldsymbol{a}_{0}, \frac{\partial \boldsymbol{v}}{\partial t}(0)=\boldsymbol{b}_{0}, \\
& v_{3}, \theta \in C\left([0, T] ; L^{2}\left(\omega ; H_{0}^{1}(0, L)\right)\right) C^{1}\left([0, T] ; L^{2}(\Omega)\right), \\
& \theta(0)=0, \frac{\partial \theta}{\partial t}(0)=0 .
\end{aligned}
$$

In order to prove that the variational problem (7.21) is equivalent to (2.17), we integrate (7.21) with respect to $y$ over $B$. Since $\boldsymbol{u}_{0}=\boldsymbol{v}+\theta \boldsymbol{e}_{3} \wedge\left(\boldsymbol{y}-\boldsymbol{y}_{B}\right)$ and 
$\boldsymbol{w}_{0}=\boldsymbol{\psi}+\varphi \boldsymbol{e}_{3} \wedge\left(\boldsymbol{y}-\boldsymbol{y}_{B}\right)$ in $\Omega \times(0, T) \times B$, taking (2.12) into account, we obtain

$$
\begin{aligned}
& \int_{\Omega \times(0, T) \times(Y \backslash B)} \rho \boldsymbol{u}_{0} \cdot \frac{\partial^{2} \boldsymbol{w}_{0}}{\partial t^{2}} d x d t d y+\int_{\Omega \times(Y \backslash B)} \rho \boldsymbol{a}_{0} \cdot \frac{\partial \boldsymbol{w}_{0}}{\partial t}(0) d x d y \\
& -\int_{\Omega \times(Y \backslash B)} \rho \boldsymbol{b}_{0} \cdot \boldsymbol{w}_{0}(0) d x d y+\int_{\Omega \times(0, T) \times(Y \backslash B)} \boldsymbol{e}_{y}\left(\boldsymbol{u}_{0}\right): \boldsymbol{\sigma}_{0 y}\left(\boldsymbol{w}_{0}\right) d x d t d y \\
& +\int_{\Omega \times(0, T)} \bar{\rho}_{1}\left(\boldsymbol{v}+\theta \boldsymbol{e}_{3} \wedge\left(\boldsymbol{y}_{G}-\boldsymbol{y}_{B}\right)\right) \cdot \frac{\partial^{2} \boldsymbol{\psi}}{\partial t^{2}} d x d t+\int_{\Omega} \bar{\rho}_{1} \boldsymbol{a}_{0} \cdot \frac{\partial \boldsymbol{\psi}}{\partial t}(0) d x \\
& -\int_{\Omega} \bar{\rho}_{1} \boldsymbol{b}_{0} \cdot \boldsymbol{\psi}(0) d x+k|B| \frac{3 l+2}{l+1} \int_{\Omega \times(0, T)} \frac{\partial v_{3}}{\partial x_{3}} \frac{\partial \psi_{3}}{\partial x_{3}} d x d t \\
& +\int_{\Omega \times(0, T)}\left(J^{\rho} \theta+\bar{\rho}_{1}\left(\left(\boldsymbol{y}_{G}-\boldsymbol{y}_{B}\right) \wedge \boldsymbol{v}\right) \cdot \boldsymbol{e}_{3}\right) \frac{\partial^{2} \varphi}{\partial t^{2}} d x d t \\
& +\int_{\Omega} \bar{\rho}_{1}\left(\left(\boldsymbol{y}_{G}-\boldsymbol{y}_{B}\right) \wedge \boldsymbol{a}_{0}\right) \cdot \boldsymbol{e}_{3} \frac{\partial \varphi}{\partial t}(0) d x-\int_{\Omega} \bar{\rho}_{1}\left(\left(\boldsymbol{y}_{G}-\boldsymbol{y}_{B}\right) \wedge \boldsymbol{b}_{0}\right) \cdot \boldsymbol{e}_{3} \varphi(0) d x \\
& +k J \int_{\Omega \times(0, T)} \frac{\partial \theta}{\partial x_{3}} \frac{\partial \varphi}{\partial x_{3}} d x d t=\int_{\Omega \times(0, T) \times(Y \backslash B)} \rho \boldsymbol{f} \cdot \boldsymbol{w}_{0} d x d t d y \\
& +\int_{\Omega \times(0, T)} \bar{\rho}_{1} \boldsymbol{f} \cdot \boldsymbol{\psi} d x d t+\int_{\Omega \times(0, T)} \bar{\rho}_{1}\left(\left(\boldsymbol{y}_{G}-\boldsymbol{y}_{B}\right) \wedge \boldsymbol{f}\right) \cdot \boldsymbol{e}_{3} \varphi d x d t .
\end{aligned}
$$

Choosing

$$
\psi=0, \quad \varphi=0
$$

noticing that $\boldsymbol{e}_{y}\left(\boldsymbol{u}_{0}\right): \boldsymbol{\sigma}_{0 y}\left(\boldsymbol{w}_{0}\right)=\boldsymbol{\sigma}_{0 y}\left(\boldsymbol{u}_{0}\right): \boldsymbol{\nabla}_{y}\left(\boldsymbol{w}_{0}\right)$, we find

$$
\begin{gathered}
\int_{\Omega \times(0, T) \times(Y \backslash B)} \rho \boldsymbol{u}_{0} \cdot \frac{\partial^{2} \boldsymbol{w}_{0}}{\partial t^{2}} d x d t d y+\int_{\Omega \times(Y \backslash B)} \rho \boldsymbol{a}_{0} \cdot \frac{\partial \boldsymbol{w}_{0}}{\partial t}(0) d x d y \\
-\int_{\Omega \times(Y \backslash B)} \rho \boldsymbol{b}_{0} \cdot \boldsymbol{w}_{0}(0) d x d y+\int_{\Omega \times(0, T) \times(Y \backslash B)} \boldsymbol{\sigma}_{0 y}\left(\boldsymbol{u}_{0}\right): \nabla_{y}\left(\boldsymbol{w}_{0}\right) d x d t d y \\
=\int_{\Omega \times(0, T) \times(Y \backslash B)} \rho \boldsymbol{f} \cdot \boldsymbol{w}_{0} d x d t d y,
\end{gathered}
$$

and, letting $\boldsymbol{w}_{0}$ vary over $\mathcal{D}\left(\Omega \times(0, T) \times(Y \backslash B) ; \mathbb{R}^{3}\right)$, deduce

$$
\rho \frac{\partial^{2} \boldsymbol{u}_{0}}{\partial t^{2}}-\operatorname{div}_{y}\left(\boldsymbol{\sigma}_{0 y}\left(\boldsymbol{u}_{0}\right)\right)=\rho \boldsymbol{f} \quad \text { in } \Omega \times(0, T) \times(Y \backslash B) .
$$

By integrating (7.29) by parts for an arbitrary $\boldsymbol{w}_{0}$ satisfying (7.2), (7.3), (7.28), we infer from (7.30) that $\int_{\Omega \times(0, T) \times \partial Y} \boldsymbol{\sigma}_{0 y}\left(\boldsymbol{u}_{0}\right) . \boldsymbol{n} . \boldsymbol{w}_{0} d x d t d \mathcal{H}^{1}(y)=0$ (n:= outward pointing normal to $\partial Y)$. Noticing that by (6.22) there holds $\boldsymbol{\sigma}_{0 y}\left(\boldsymbol{u}_{0}\right) \cdot \boldsymbol{n}=0$ on $\partial Y \cap \bar{B}$, we deduce

$$
\boldsymbol{\sigma}_{0 y}\left(\boldsymbol{u}_{0}\right) \cdot \boldsymbol{n}(x, t, y)=-\boldsymbol{\sigma}_{0 y}\left(\boldsymbol{u}_{0}\right) \cdot \boldsymbol{n}(x, t,-y) \quad \text { on } \quad \Omega \times(0, T) \times \partial Y .
$$

Fixing $\left(\boldsymbol{w}_{0}, \boldsymbol{\psi}, \varphi\right) \in L^{2}(0, T ; H)$ satisfying (7.2), (7.3), we infer from (2.3), (7.1), (7.31) 
$\left(\boldsymbol{n}=-\boldsymbol{n}_{B}\right)$ that

$$
\begin{aligned}
& -\int_{\Omega \times(0, T) \times \partial(Y \backslash B)} \boldsymbol{\sigma}_{0 y}\left(\boldsymbol{u}_{0}\right) \cdot \boldsymbol{n} \cdot \boldsymbol{w}_{0} d x d t d \mathcal{H}^{1}(y) \\
& \quad=\int_{\Omega \times(0, T) \times \partial B} \boldsymbol{\sigma}_{0 y}\left(\boldsymbol{u}_{0}\right) \cdot \boldsymbol{n}_{B} \cdot\left(\boldsymbol{\psi}+\varphi \boldsymbol{e}_{3} \wedge\left(\boldsymbol{y}-\boldsymbol{y}_{B}\right)\right) d x d t d \mathcal{H}^{1}(y) \\
& \quad=\int_{\Omega \times(0, T)}\left(\boldsymbol{g}\left(\boldsymbol{u}_{0}\right) \cdot \boldsymbol{\psi}+\boldsymbol{m}\left(\boldsymbol{u}_{0}\right) \cdot \boldsymbol{e}_{3} \varphi\right) d x d t .
\end{aligned}
$$

By multiplying (7.30) by $\boldsymbol{w}_{0}$ and by integrating it by parts over $\Omega \times(0, T) \times(Y \backslash B)$, thanks to $(7.26),(7.31),(7.32)$ we obtain

$$
\begin{aligned}
& \int_{\Omega \times(0, T) \times(Y \backslash B)} \rho \boldsymbol{u}_{0} \cdot \frac{\partial^{2} \boldsymbol{w}_{0}}{\partial t^{2}} d x d t d y+\int_{\Omega \times(Y \backslash B)} \rho \boldsymbol{a}_{0} \cdot \frac{\partial \boldsymbol{w}_{0}}{\partial t}(0) d x d y \\
& -\int_{\Omega \times(Y \backslash B)} \rho \boldsymbol{b}_{0} \cdot \boldsymbol{w}_{0}(0) d x d y+\int_{\Omega \times(0, T) \times(Y \backslash B)} \boldsymbol{e}_{y}\left(\boldsymbol{u}_{0}\right): \boldsymbol{\sigma}_{0 y}\left(\boldsymbol{w}_{0}\right) d x d t d y \\
& \quad+\int_{\Omega \times(0, T)}\left(\boldsymbol{g}\left(\boldsymbol{u}_{0}\right) \boldsymbol{\psi}+\boldsymbol{m}\left(\boldsymbol{u}_{0}\right) \cdot \boldsymbol{e}_{3} \varphi\right) d x d t=\int_{\Omega \times(0, T) \times(Y \backslash B)}^{\rho f \cdot \boldsymbol{w}_{0} d x d t d y}
\end{aligned}
$$

By subtracting (7.33) from (7.27), we find

$$
\begin{aligned}
& \int_{\Omega \times(0, T)} \bar{\rho}_{1}\left(\boldsymbol{v}+\theta \boldsymbol{e}_{3} \wedge\left(\boldsymbol{y}_{G}-\boldsymbol{y}_{B}\right)\right) \cdot \frac{\partial^{2} \boldsymbol{\psi}}{\partial t^{2}} d x d t \\
& -\int_{\Omega \times(0, T)} \boldsymbol{g}\left(\boldsymbol{u}_{0}\right) \cdot \boldsymbol{\psi} d x d t+k|B| \int_{\Omega \times(0, T)} \frac{3 l+2}{l+1} \frac{\partial v_{3}}{\partial x_{3}} \frac{\partial \psi_{3}}{\partial x_{3}} d x d t \\
& +\int_{\Omega \times(0, T)}\left(J^{\rho} \theta+\bar{\rho}_{1}\left(\left(\boldsymbol{y}_{G}-\boldsymbol{y}_{B}\right) \wedge \boldsymbol{v}\right) \cdot \boldsymbol{e}_{3}\right) \frac{\partial^{2} \varphi}{\partial t^{2}} d x d t-\int_{\Omega \times(0, T)} \boldsymbol{m}\left(\boldsymbol{u}_{0}\right) \cdot \boldsymbol{e}_{3} \varphi d x d t \\
& +k J \int_{\Omega \times(0, T)} \frac{\partial \theta}{\partial x_{3}} \frac{\partial \varphi}{\partial x_{3}} d x d t+\int_{\Omega} \bar{\rho}_{1} \boldsymbol{a}_{0} \cdot \frac{\partial \boldsymbol{\psi}}{\partial t}(0) d x-\int_{\Omega} \bar{\rho}_{1} \boldsymbol{b}_{0} \cdot \boldsymbol{\psi}(0) d x \\
& -\int_{\Omega} \bar{\rho}_{1}\left(\left(\boldsymbol{y}_{G}-\boldsymbol{y}_{B}\right) \wedge \boldsymbol{b}_{0}\right) \cdot \boldsymbol{e}_{3} \varphi(0) d x+\int_{\Omega} \bar{\rho}_{1}\left(\left(\boldsymbol{y}_{G}-\boldsymbol{y}_{B}\right) \wedge \boldsymbol{a}_{0}\right) \cdot \boldsymbol{e}_{3} \frac{\partial \varphi}{\partial t}(0) d x \\
& \quad=\int_{\Omega \times(0, T)} \bar{\rho}_{1} \boldsymbol{f} \cdot \boldsymbol{\psi} d x d t+\int_{\Omega \times(0, T)} \bar{\rho}_{1}\left(\left(\boldsymbol{y}_{G}-\boldsymbol{y}_{B}\right) \wedge \boldsymbol{f}\right) \cdot \boldsymbol{e}_{3} \varphi d x d t .
\end{aligned}
$$

Making $(\boldsymbol{\psi}, \varphi)$ vary in $\mathcal{D}\left(\Omega \times(0, T) ; \mathbb{R}^{3}\right) \times \mathcal{D}(\Omega \times(0, T))$, we infer

$$
\begin{gathered}
\bar{\rho}_{1} \frac{\partial^{2} \boldsymbol{v}}{\partial t^{2}}+\bar{\rho}_{1} \frac{\partial^{2} \theta}{\partial t^{2}} \boldsymbol{e}_{3} \wedge\left(\boldsymbol{y}_{G}-\boldsymbol{y}_{B}\right)-k|B| \frac{3 l+2}{l+1} \frac{\partial^{2} v_{3}}{\partial x_{3}^{2}} \boldsymbol{e}_{3}= \\
\bar{\rho}_{1} \boldsymbol{f}+\boldsymbol{g}\left(\boldsymbol{u}_{0}\right) \text { in } \Omega \times(0, T), \\
\bar{\rho}_{1}\left(\left(\boldsymbol{y}_{G}-\boldsymbol{y}_{B}\right) \wedge \frac{\partial^{2} \boldsymbol{v}}{\partial t^{2}}\right) \cdot \boldsymbol{e}_{3}+J^{\rho} \frac{\partial^{2} \theta}{\partial t^{2}}-k J \frac{\partial^{2} \theta}{\partial x_{3}^{2}}= \\
\bar{\rho}_{1}\left(\left(\boldsymbol{y}_{G}-\boldsymbol{y}_{B}\right) \wedge \boldsymbol{f}\right) \cdot \boldsymbol{e}_{3}+\boldsymbol{m}\left(\boldsymbol{u}_{0}\right) \cdot \boldsymbol{e}_{3} \quad \text { in } \Omega \times(0, T) .
\end{gathered}
$$

By (6.22), (7.26), (7.30), (7.31), (7.34), the triple $\left(\boldsymbol{u}_{0}, \boldsymbol{v}, \theta\right)$ is a solution of (2.17), (2.19). Conversely, any solution of (2.17), (2.19) satisfies (7.21).

Case $k=+\infty, \kappa=0$. By (2.9), (2.10) we have

$$
\lim _{\varepsilon \rightarrow 0} \mu_{1 \varepsilon}=+\infty, \quad \lim _{\varepsilon \rightarrow 0} \varepsilon^{2} \mu_{1 \varepsilon}=0, \quad \boldsymbol{a}_{0}=0 .
$$


We consider again the sequence $\left(\boldsymbol{\chi}_{\varepsilon}\right)$ defined by (7.17), and assume now that

$$
\varphi=0, \quad \psi_{3}=0
$$

yielding by (7.18), $\left|\boldsymbol{\sigma}_{\varepsilon}\left(\phi_{\varepsilon}\right) 1_{B_{\varepsilon}}\right| \leq C \mu_{1 \varepsilon} \varepsilon$. Taking (7.12), (7.35) and the estimate $\int_{B_{\varepsilon} \times(0, T)}\left|\boldsymbol{e}\left(\boldsymbol{u}_{\varepsilon}\right)\right|^{2} d x d t \leq \frac{C}{\mu_{1 \varepsilon}}$ (see (6.20)) into account, we deduce

$$
\begin{aligned}
\limsup _{\varepsilon \rightarrow 0} I_{3 \varepsilon} & \leq C \limsup _{\varepsilon \rightarrow 0} \mu_{1 \varepsilon} \varepsilon \sqrt{\int_{B_{\varepsilon} \times(0, T)}\left|\boldsymbol{e}\left(\boldsymbol{u}_{\varepsilon}\right)\right|^{2} d x d t} \\
& \leq C \limsup _{\varepsilon \rightarrow 0} \varepsilon \sqrt{\mu_{1 \varepsilon}}=0 .
\end{aligned}
$$

By (7.11), (7.12), (7.14), (7.16), (7.35), (7.37), passing to the limit as $\varepsilon \rightarrow 0$ in (7.8) we obtain

$$
\begin{aligned}
& \int_{\Omega \times(0, T) \times Y} \rho \boldsymbol{u}_{0} \cdot \frac{\partial^{2} \boldsymbol{w}_{0}}{\partial t^{2}} d x d t d y-\int_{\Omega \times Y} \rho \boldsymbol{b}_{0} \cdot \boldsymbol{w}_{0}(0) d x d y \\
& \quad+\int_{\Omega \times(0, T) \times(Y \backslash B)} \boldsymbol{e}_{y}\left(\boldsymbol{u}_{0}\right): \boldsymbol{\sigma}_{0 y}\left(\boldsymbol{w}_{0}\right) d x d t d y=\int_{\Omega \times(0, T) \times Y} \rho \boldsymbol{f} \cdot \boldsymbol{w}_{0} d x d t d y .
\end{aligned}
$$

This variational problem is equivalent to $(6.10)$, where (notice that by $(6.22),(6.23)$, (6.26), $\left.\xi=\left(\boldsymbol{u}_{0}, \boldsymbol{v}, \theta\right) \in L^{2}\left(0, T ; V^{(2)}\right), \xi^{\prime} \in L^{2}\left(0, T ; H^{(2)}\right)\right)$

$$
\begin{aligned}
& H^{(2)}:=\left\{\left(\boldsymbol{w}_{0}, \boldsymbol{\psi}, \theta\right) \in H, \boldsymbol{\psi}_{3}=\theta=0\right\} \\
& (., .)_{H^{(2)}}:=(., .)_{H}, \quad V^{(2)}:=V \cap H^{(2)},((., .))_{V^{(2)}}:=((., .))_{V}, \\
& h^{(2)}:=\left(\boldsymbol{f} 1_{Y \backslash B}+\left(f_{1} \boldsymbol{e}_{1}+f_{2} \boldsymbol{e}_{2}\right) 1_{B}, f_{1} \boldsymbol{e}_{1}+f_{2} \boldsymbol{e}_{2}, 0\right), \\
& \bar{a}^{(2)}:=0, \quad a^{(2)}:=a, \quad \xi_{0}^{(2)}:=0, \\
& \xi_{1}^{(2)}:=\left(\boldsymbol{b}_{0} 1_{Y \backslash B}+\left(\left(\boldsymbol{b}_{0}\right)_{1} \boldsymbol{e}_{1}+\left(\boldsymbol{b}_{0}\right)_{2} \boldsymbol{e}_{2}\right) 1_{B},\left(\boldsymbol{b}_{0}\right)_{1} \boldsymbol{e}_{1}+\left(\boldsymbol{b}_{0}\right)_{2} \boldsymbol{e}_{2}, 0\right),
\end{aligned}
$$

the spaces $H$ and $V$ being given by (7.1), (7.22). By (7.23), (7.39), the estimate (6.9) is satisfied. We deduce from Theorem 6.2 that $\xi=\left(\boldsymbol{u}_{0}, \boldsymbol{v}, \theta\right)$ is the unique solution of (7.38) and that $\xi \in C\left([0, T] ; V^{(2)}\right) \cap C^{1}\left([0, T] ; H^{(2)}\right), \xi(0)=0, \frac{\partial \xi}{\partial t}(0)=\xi_{1}^{(2)}$, yielding by $(7.25),(7.39)$ the initial-boundary conditions and regularity properties stated in (2.18), (2.20). By integrating (7.38) with respect to $y$ over $B$, we get

$$
\begin{aligned}
& \int_{\Omega \times(0, T) \times(Y \backslash B)} \rho \boldsymbol{u}_{0} \cdot \frac{\partial^{2} \boldsymbol{w}_{0}}{\partial t^{2}} d x d t d y-\int_{\Omega \times(Y \backslash B)} \rho \boldsymbol{b}_{0} \cdot \boldsymbol{w}_{0}(0) d x d y \\
& +\int_{\Omega \times(0, T) \times(Y \backslash B)} \boldsymbol{e}_{y}\left(\boldsymbol{u}_{0}\right): \boldsymbol{\sigma}_{0 y}\left(\boldsymbol{w}_{0}\right) d x d t d y+\int_{\Omega \times(0, T)} \bar{\rho}_{1}\left(v_{1} \frac{\partial^{2} \psi_{1}}{\partial t^{2}}+v_{2} \frac{\partial^{2} \psi_{2}}{\partial t^{2}}\right) d x d t \\
& \quad-\int_{\Omega} \bar{\rho}_{1}\left(\left(\boldsymbol{b}_{0}\right)_{1} \psi_{1}(0)+\left(\boldsymbol{b}_{0}\right)_{2} \psi_{2}(0)\right) d x \\
& \quad=\int_{\Omega \times(0, T) \times(Y \backslash B)} \rho \boldsymbol{f} \cdot \boldsymbol{w}_{0} d x d t d y+\int_{\Omega \times(0, T)} \bar{\rho}_{1}\left(f_{1} \psi_{1}+f_{2} \psi_{2}\right) d x d t .
\end{aligned}
$$

Setting $\psi_{1}=\psi_{2}=0$ in (7.40), we find (7.29) and deduce (7.30), (7.31), (7.32), (7.33) (substituting 0 for $\boldsymbol{a}_{0}, \psi_{3}, \varphi$ ). Then, substracting (7.33) from (7.40), we find

$$
\begin{aligned}
\int_{\Omega \times(0, T)} \bar{\rho}_{1}\left(v_{1} \frac{\partial^{2} \psi_{1}}{\partial t^{2}}+v_{2} \frac{\partial^{2} \psi_{2}}{\partial t^{2}}\right) d x d t-\int_{\Omega} \bar{\rho}_{1}\left(\left(\boldsymbol{b}_{0}\right)_{1} \psi_{1}(x, 0)+\left(\boldsymbol{b}_{0}\right)_{2} \psi_{2}(x, 0)\right) d x \\
=\int_{\Omega \times(0, T)}\left(\left(\boldsymbol{g}\left(\boldsymbol{u}_{0}\right)\right)_{1} \boldsymbol{\psi}_{1}+\left(\boldsymbol{g}\left(\boldsymbol{u}_{0}\right)\right)_{2} \boldsymbol{\psi}_{2}\right) d x d t+\int_{\Omega \times(0, T)} \bar{\rho}_{1}\left(f_{1} \psi_{1}+f_{2} \psi_{2}\right) d x d t
\end{aligned}
$$


Making $\psi_{1}, \psi_{2}$ vary in $\mathcal{D}(\Omega \times(0, T))$, we deduce that

$$
\begin{aligned}
& \bar{\rho}_{1} \frac{\partial^{2} v_{1}}{\partial t^{2}}=\bar{\rho}_{1} f_{1}+\left(\boldsymbol{g}\left(\boldsymbol{u}_{0}\right)\right)_{1} \text { in } \quad \Omega \times(0, T), \\
& \bar{\rho}_{1} \frac{\partial^{2} v_{2}}{\partial t^{2}}=\bar{\rho}_{1} f_{2}+\left(\boldsymbol{g}\left(\boldsymbol{u}_{0}\right)\right)_{2} \text { in } \quad \Omega \times(0, T),
\end{aligned}
$$

hence $\left(\boldsymbol{u}_{0}, \boldsymbol{v}, \theta\right)$ is solution of $(2.17),(2.20)$.

Case $0<\kappa<+\infty$. We assume (7.36), and consider the sequence $\left(\boldsymbol{\chi}_{\varepsilon}\right)$ defined by

$$
\boldsymbol{\chi}_{\varepsilon}(x, t, y):=\overline{\boldsymbol{\psi}}_{\varepsilon}(x, t)-\boldsymbol{\psi}(x, t)+\varepsilon \boldsymbol{w}_{1 \varepsilon}(x, t, y)+\varepsilon^{2} \boldsymbol{w}_{2 \varepsilon}(x, t, y),
$$

where $\overline{\boldsymbol{\psi}}_{\varepsilon}$ is given by $(6.38)$ and

$$
\begin{aligned}
\boldsymbol{w}_{1 \varepsilon}(x, t, y):=\left(-\frac{\partial \bar{\psi}_{\varepsilon 1}}{\partial x_{3}}\left(\boldsymbol{y}-\boldsymbol{y}_{B}\right)_{1}-\frac{\partial \bar{\psi}_{\varepsilon 2}}{\partial x_{3}}\left(\boldsymbol{y}-\boldsymbol{y}_{B}\right)_{2}\right) \boldsymbol{e}_{3} \\
\boldsymbol{w}_{2 \varepsilon}(x, t, y):=\frac{l_{\varepsilon}}{2\left(l_{\varepsilon}+1\right)}\left(\begin{array}{c}
\frac{\partial^{2} \bar{\psi}_{\varepsilon 1}}{\partial x_{3}^{2}} \frac{\left(\boldsymbol{y}-\boldsymbol{y}_{B}\right)_{1}^{2}-\left(\boldsymbol{y}-\boldsymbol{y}_{B}\right)_{2}^{2}}{2}+\frac{\partial^{2} \bar{\psi}_{\varepsilon 2}}{\partial x_{3}^{2}}\left(\boldsymbol{y}-\boldsymbol{y}_{B}\right)_{1}\left(\boldsymbol{y}-\boldsymbol{y}_{B}\right)_{2} \\
\frac{\partial^{2} \bar{\psi}_{\varepsilon 2}}{\partial x_{3}^{2}} \frac{\left(\boldsymbol{y}-\boldsymbol{y}_{B}\right)_{2}^{2}-\left(\boldsymbol{y}-\boldsymbol{y}_{B}\right)_{1}^{2}}{2}+\frac{\partial^{2} \bar{\psi}_{\varepsilon 1}}{\partial x_{3}^{2}}\left(\boldsymbol{y}-\boldsymbol{y}_{B}\right)_{1}\left(\boldsymbol{y}-\boldsymbol{y}_{B}\right)_{2} \\
0
\end{array}\right) .
\end{aligned}
$$

By (7.7) and (7.42) we have $\boldsymbol{\phi}_{\varepsilon}=\overline{\boldsymbol{\psi}}_{\varepsilon}(x, t)+\varepsilon \boldsymbol{w}_{1 \varepsilon}\left(x, t,\left[\frac{\boldsymbol{x}}{\varepsilon}\right]\right)+\varepsilon^{2} \boldsymbol{w}_{2 \varepsilon}\left(x, t,\left[\frac{\boldsymbol{x}}{\varepsilon}\right]\right)$ in $B_{\varepsilon}$. We deduce

$$
\begin{aligned}
\varepsilon \boldsymbol{\sigma}_{\varepsilon}\left(\boldsymbol{\phi}_{\varepsilon}\right) 1_{B_{\varepsilon}}=-\varepsilon^{2} \mu_{1 \varepsilon} \frac{3 l_{\varepsilon}+2}{l_{\varepsilon}+1}\left(\sum_{\alpha=1}^{2} \frac{\partial^{2} \bar{\psi}_{\varepsilon \alpha}}{\partial x_{3}^{2}}\left(\left[\frac{x_{\alpha}}{\varepsilon}\right]-\left(\boldsymbol{y}_{B}\right)_{\alpha}\right)\right) \boldsymbol{e}_{3} \otimes \boldsymbol{e}_{3} 1_{B_{\varepsilon}} \\
+\varepsilon^{3} \mu_{1 \varepsilon}\left(l_{\varepsilon} \operatorname{tr} \boldsymbol{e}_{y}\left(\boldsymbol{w}_{2 \varepsilon}\right)\left(x, t, \frac{x}{\varepsilon}\right) \boldsymbol{I}+2 \boldsymbol{e}_{y}\left(\boldsymbol{w}_{2 \varepsilon}\right)\left(x, t, \frac{x}{\varepsilon}\right)\right) 1_{B_{\varepsilon}}, \\
\longrightarrow-\kappa \frac{3 l+2}{l+1}\left(\sum_{\alpha=1}^{2} \frac{\partial^{2} \psi_{\alpha}}{\partial x_{3}^{2}}\left(\boldsymbol{y}-\boldsymbol{y}_{B}\right)_{\alpha}\right) 1_{B}(y) \boldsymbol{e}_{3} \otimes \boldsymbol{e}_{3},
\end{aligned}
$$

and infer from (2.2), (6.24), (6.25), (7.12) that

$$
\begin{aligned}
\lim _{\varepsilon \rightarrow 0} I_{3 \varepsilon} & =-\kappa \frac{3 l+2}{l+1} \int_{\Omega \times(0, T) \times B}\left(\frac{\partial \xi}{\partial x_{3}}-\sum_{\alpha=1}^{2} \frac{\partial^{2} v_{\alpha}}{\partial x_{3}^{2}}\left(\boldsymbol{y}-\boldsymbol{y}_{B}\right)_{\alpha}\right) \\
& \left(\sum_{\alpha=1}^{2} \frac{\partial^{2} \psi_{\alpha}}{\partial x_{3}^{2}}\left(\boldsymbol{y}-\boldsymbol{y}_{B}\right)_{\alpha}\right) d x d t d y_{1} d y_{2} \\
& =\sum_{\alpha, \beta=1}^{2} \kappa \frac{3 l+2}{l+1} J_{\alpha \beta} \int_{\Omega \times(0, T)} \frac{\partial^{2} \psi_{\alpha}}{\partial x_{3}^{2}} \frac{\partial^{2} v_{\beta}}{\partial x_{3}^{2}} d x d t
\end{aligned}
$$

Passing to the limit in (7.8), by (2.10), (7.11), (7.12), (7.14), (7.16), (7.36), (7.43), we get

$$
\begin{aligned}
& \int_{\Omega \times(0, T) \times Y} \rho \boldsymbol{u}_{0} \cdot \frac{\partial^{2} \boldsymbol{w}_{0}}{\partial t^{2}} d x d t d y \\
& -\int_{\Omega \times Y} \rho \boldsymbol{b}_{0} \cdot \boldsymbol{w}_{0}(0) d x d y+\int_{\Omega \times(0, T) \times(Y \backslash B)} \boldsymbol{e}_{y}\left(\boldsymbol{u}_{0}\right): \boldsymbol{\sigma}_{0}\left(\boldsymbol{w}_{0}\right) d x d t d y \\
& +\sum_{\alpha, \beta=1}^{2} \kappa \frac{3 l+2}{l+1} J_{\alpha \beta} \int_{\Omega \times(0, T)} \frac{\partial^{2} \psi_{\alpha}}{\partial x_{3}^{2}} \frac{\partial^{2} v_{\beta}}{\partial x_{3}^{2}} d x d t=\int_{\Omega \times(0, T) \times Y} \rho \boldsymbol{f} \cdot \boldsymbol{w}_{0} d x d t d y,
\end{aligned}
$$


for all $\left(\boldsymbol{w}_{0}, \boldsymbol{\psi}, \varphi\right) \in L^{2}(0, T ; H)$ satisfying (7.2), (7.3), (7.36). We set (see (7.22), $(7.39))$

$$
\begin{aligned}
& H^{(3)}:=H^{(2)}, \quad V^{(3)}:=\left\{\left(\boldsymbol{w}_{0}, \boldsymbol{\psi}, \varphi\right) \in V^{(2)}, \psi_{1}, \psi_{2} \in L^{2}\left(\omega ; H_{0}^{2}(0, L)\right)\right\}, \\
& \left(\left(\left(\boldsymbol{u}_{0}, \boldsymbol{v}, \theta\right),\left(\boldsymbol{w}_{0}, \boldsymbol{\psi}, \varphi\right)\right)\right)_{V^{(3)}}:=\left(\left(\left(\boldsymbol{u}_{0}, \boldsymbol{v}, \theta\right),\left(\boldsymbol{w}_{0}, \boldsymbol{\psi}, \varphi\right)\right)\right)_{V} \\
& +\int_{\Omega}\left(\frac{\partial^{2} v_{1}}{\partial x_{3}^{2}} \frac{\partial^{2} \psi_{1}}{\partial x_{3}^{2}}+\frac{\partial^{2} v_{2}}{\partial x_{3}^{2}} \frac{\partial^{2} \psi_{2}}{\partial x_{3}^{2}}\right) d x \\
& (7.45) \bar{a}^{(3)}((\boldsymbol{v}, \theta),(\boldsymbol{\psi}, \varphi)):=\sum_{\alpha, \beta=1}^{2} \kappa \frac{3 l+2}{l+1} J_{\alpha \beta} \int_{\Omega} \frac{\partial^{2} \psi_{\alpha}}{\partial x_{3}^{2}} \frac{\partial^{2} v_{\beta}}{\partial x_{3}^{2}} d x, \\
& a^{(3)}\left(\left(\boldsymbol{u}_{0}, \boldsymbol{v}, \theta\right),\left(\boldsymbol{w}_{0}, \boldsymbol{\psi}, \varphi\right)\right):=\int_{\Omega \times(Y \backslash B)} \boldsymbol{e}_{y}\left(\boldsymbol{u}_{0}\right): \boldsymbol{\sigma}_{y}\left(\boldsymbol{w}_{0}\right) d x d y \\
& +\bar{a}^{(3)}((\boldsymbol{v}, \theta),(\boldsymbol{\psi}, \varphi)), \\
& \left(\xi_{0}^{(3)}, \xi_{1}^{(3)}, h^{(3)}\right):=\left(\xi_{0}^{(2)}, \xi_{1}^{(2)}, h^{(2)}\right) .
\end{aligned}
$$

By (6.22), (6.23), (6.25), (6.26), there holds $\xi=\left(\boldsymbol{u}_{0}, \boldsymbol{v}, \theta\right) \in L^{2}\left(0, T ; V^{(3)}\right), \xi^{\prime} \in$ $L^{2}\left(0, T ; H^{(3)}\right)$, therefore the variational formulation (7.44) is equivalent to (6.10). We check that the eigenvalues of the $2 \times 2$ symmetric matrix of $(\alpha, \beta)^{t h}$ entries $J_{\alpha \beta}$ given by (2.2) are positive, and deduce that $\sum_{\alpha, \beta=1}^{2} J_{\alpha \beta} s_{\alpha} s_{\beta} \geq c|s|_{\mathbb{R}^{2}}^{2}, \forall \boldsymbol{s} \in \mathbb{R}^{2}$, for a suitable $c>0$. Taking (7.22), (7.23) and (7.45) into account, we infer

$$
\begin{aligned}
\|\tilde{\xi}\|_{V^{(3)}}^{2} & \leq\|\tilde{\xi}\|_{V}^{2}+C \bar{a}^{(3)}((\boldsymbol{\psi}, \varphi),(\boldsymbol{\psi}, \varphi)) \\
& \leq C\left(|\tilde{\xi}|_{H}+a(\tilde{\xi}, \tilde{\xi})+\bar{a}^{(3)}((\boldsymbol{\psi}, \varphi),(\boldsymbol{\psi}, \varphi))\right) \\
& \leq C\left(|\tilde{\xi}|_{H^{(3)}}+a^{(3)}(\tilde{\xi}, \tilde{\xi})\right),
\end{aligned}
$$

that is (6.9). We deduce from Theorem 6.2 that $\xi=\left(\boldsymbol{u}_{0}, \boldsymbol{v}, \theta\right)$ is the unique solution of (7.44) and that $\xi \in C\left([0, T] ; V^{(3)}\right) \cap C^{1}\left([0, T] ; H^{(3)}\right), \xi(0)=0, \frac{\partial \xi}{\partial t}(0)=\xi_{1}^{(3)}$, yielding by (7.25) and the inequality $\sum_{\alpha=1}^{2}\left\|\psi_{\alpha}\right\|_{L^{2}\left(\omega ; H_{0}^{2}(0, L)\right)} \leq C\left\|\left(\boldsymbol{w}_{0}, \boldsymbol{\psi}, \varphi\right)\right\|_{V^{(3)}}, \forall\left(\boldsymbol{w}_{0}, \boldsymbol{\psi}, \varphi\right) \in$ $V^{(3)}$, the initial-boundary conditions and regularity properties stated in (2.18), (2.21). Repeating the argument of the case $0<k<+\infty$, we integrate (7.44) with respect to $y$ over $B$, set $\psi_{1}=\psi_{2}=0$, find (7.29), deduce (7.30), (7.31), (7.32), (7.33), subtract (7.33) from (7.44), get

$$
\begin{aligned}
\int_{\Omega \times(0, T)} \bar{\rho}_{1} \boldsymbol{v} & \frac{\partial^{2} \boldsymbol{\psi}}{\partial t^{2}} d x d t+\int_{\Omega} \bar{\rho}_{1} \boldsymbol{b}_{0} \cdot \boldsymbol{\psi}(0) d x-\int_{\Omega \times(0, T)} \boldsymbol{g}\left(\boldsymbol{u}_{0}\right) \cdot \boldsymbol{\psi} d x d t \\
& +\sum_{\alpha, \beta=1}^{2} \kappa \frac{3 l+2}{l+1} J_{\alpha \beta} \int_{\Omega \times(0, T)} \frac{\partial^{2} \psi_{\alpha}}{\partial x_{3}^{2}} \frac{\partial^{2} v_{\beta}}{\partial x_{3}^{2}} d x d t=\int_{\Omega \times(0, T)} \bar{\rho}_{1} \boldsymbol{f} \cdot \boldsymbol{\psi} d x d t
\end{aligned}
$$

then, making $\psi_{1}, \psi_{2}$ vary in $\mathcal{D}(\Omega \times(0, T))$, infer $\bar{\rho}_{1} \frac{\partial^{2} v_{\alpha}}{\partial t^{2}}(x, t)+\sum_{\beta=1}^{2} \kappa \frac{3 l+2}{l+1} J_{\alpha \beta} \frac{\partial^{4} v_{\beta}}{\partial x_{3}^{4}}=$ $\bar{\rho}_{1} f_{\alpha}+\left(\boldsymbol{g}\left(\boldsymbol{u}_{0}\right)\right)_{\alpha}$, in $\Omega \times(0, T)$ for $\alpha \in\{1,2\}$, and deduce that $\left(\boldsymbol{u}_{0}, \boldsymbol{v}, \theta\right)$ satisfies (2.17), (2.21).

Case $\kappa=+\infty$. We set

$$
\chi_{\varepsilon}=0, \quad \psi=0, \quad \varphi=0 .
$$

By (7.7), (7.12), we have $I_{3 \varepsilon}=0$. By passing to the limit as $\varepsilon \rightarrow 0$ in (7.8), we obtain the variational problem (7.29) and deduce that $\left(\boldsymbol{u}_{0}, \boldsymbol{v}, \theta\right)$ satisfies $(2.17),(2.22)$. 
Proof of the corrector result (2.25). We consider the fibered case, when $0<k<+\infty$ (the other cases are similar). Setting (7.22), we introduce the continuous symmetric bilinear form on $W^{1,2}(0, T ; V, H):=\left\{\zeta \in L^{2}(0, T ; V), \zeta^{\prime} \in L^{2}(0, T ; H)\right\}$ defined by

$$
\tilde{a}(\zeta, \tilde{\zeta}):=\int_{0}^{T}\left(\left(\zeta^{\prime}, \tilde{\zeta}^{\prime}\right)_{H}+a(\zeta, \tilde{\zeta})\right) d t, \quad \forall(\zeta, \tilde{\zeta}) \in\left(W^{1,2}(0, T ; V, H)\right)^{2}
$$

We fix $\tilde{\xi}:=\left(\boldsymbol{w}_{0}, \boldsymbol{\psi}, \varphi\right) \in W^{1,2}(0, T ; V, H)$ satisfying (7.2) (not (7.3)) and set (7.7). There holds $\phi_{\varepsilon} \in C\left([0, T] ; H_{0}^{1}\left(\Omega ; \mathbb{R}^{3}\right)\right) \cap C^{1}\left([0, T] ; L^{2}\left(\Omega ; \mathbb{R}^{3}\right)\right)$ for small epsilons. By applying (6.14) to $\boldsymbol{w}=\boldsymbol{u}_{\varepsilon}-\boldsymbol{\phi}_{\varepsilon}$ and by integrating it over $(0, T)$, taking (2.23) into account, we infer

$$
\begin{aligned}
& \int_{\Omega \times(0, T)}\left|\boldsymbol{u}_{\varepsilon}-\phi_{\varepsilon}\right|^{2} d x d t \leq C\left(J_{1 \varepsilon}-2 J_{2 \varepsilon}+J_{3 \varepsilon}\right)+C \int_{\Omega \times(0, T)}\left|\phi_{\varepsilon}(0)\right|^{2} d x d t, \\
& J_{1 \varepsilon}:=\int_{\Omega \times(0, T)} \rho_{\varepsilon}\left|\frac{\partial \boldsymbol{u}_{\varepsilon}}{\partial t}\right|^{2}+\boldsymbol{e}\left(\boldsymbol{u}_{\varepsilon}\right): \boldsymbol{\sigma}_{\varepsilon}\left(\boldsymbol{u}_{\varepsilon}\right) d x d t \\
& J_{2 \varepsilon}:=\int_{\Omega \times(0, T)} \rho_{\varepsilon} \frac{\partial \boldsymbol{u}_{\varepsilon}}{\partial t} \cdot \frac{\partial \phi_{\varepsilon}}{\partial t}+\boldsymbol{e}\left(\boldsymbol{u}_{\varepsilon}\right): \boldsymbol{\sigma}_{\varepsilon}\left(\boldsymbol{\phi}_{\varepsilon}\right) d x d t \\
& J_{3 \varepsilon}:=\int_{\Omega \times(0, T)} \rho_{\varepsilon}\left|\frac{\partial \phi_{\varepsilon}}{\partial t}\right|^{2}+\boldsymbol{e}\left(\phi_{\varepsilon}\right): \boldsymbol{\sigma}_{\varepsilon}\left(\boldsymbol{\phi}_{\varepsilon}\right) d x d t .
\end{aligned}
$$

In order to compute the limit of $\left(J_{1 \varepsilon}\right)$, we notice that by $(2.23)$ and $(6.27)$ we have $\boldsymbol{a}_{0}=0$ and

$$
J_{1 \varepsilon}=\int_{\Omega \times(0, T)} \rho_{\varepsilon}\left|\boldsymbol{b}_{0}\right|^{2} d x d t+2 \int_{0}^{T}\left(\int_{\Omega \times(0, t)} \rho_{\varepsilon} f \cdot \frac{\partial \boldsymbol{u}_{\varepsilon}}{\partial t} d x d s\right) d t .
$$

Since $\rho_{\varepsilon} 1_{\Omega \times(0, t)} \longrightarrow \rho 1_{(0, t)}$ for all $t \in(0, T)$ and since, by (6.20), $\mid \int_{\Omega \times(0, t)} \rho_{\varepsilon} \boldsymbol{f} \cdot \frac{\partial \boldsymbol{u}_{\varepsilon}}{\partial t}$ $d x d s \mid \leq C$, we deduce from (6.26), (7.22) and from the Dominated Convergence Theorem that

$$
\begin{aligned}
\lim _{\varepsilon \rightarrow 0} J_{1 \varepsilon} & =\int_{\Omega \times(0, T) \times Y} \rho\left|\boldsymbol{b}_{0}\right|^{2} d x d y d t+2 \int_{0}^{T}\left(\int_{\Omega \times(0, t) \times Y} \rho \boldsymbol{f} \cdot \frac{\partial \boldsymbol{u}_{0}}{\partial t} d x d s d y\right) d t \\
& =\int_{0}^{T}\left(\left(\xi_{1}, \xi_{1}\right)_{H}+2 \int_{0}^{t}\left(h, \xi^{\prime}\right)_{H} d s\right) d t \\
& =2 \int_{0}^{T}\left(e(0)+\int_{0}^{t}\left(h, \xi^{\prime}\right)_{H} d s\right) d t .
\end{aligned}
$$

Applying the energy equation (6.12), taking (7.47) into account, we infer

$$
\lim _{\varepsilon \rightarrow 0} J_{1 \varepsilon}=2 \int_{0}^{T} e(t) d t=\int_{0}^{T}\left(\left(\xi^{\prime}, \xi^{\prime}\right)_{H}+a(\xi, \xi)\right) d t=\tilde{a}(\xi, \xi) .
$$

By (6.26), (7.10), (7.12), (7.14), (7.16), (7.20), (7.22) we have $\lim _{\varepsilon \rightarrow 0} \int_{\Omega \times(0, T)} \boldsymbol{e}\left(\boldsymbol{u}_{\varepsilon}\right)$ : $\boldsymbol{\sigma}_{\varepsilon}\left(\phi_{\varepsilon}\right) d x d t=\int_{0}^{T} a(\xi, \tilde{\xi}) d t$, and $\lim _{\varepsilon \rightarrow 0} \int_{\Omega \times(0, T)} \rho_{\varepsilon} \frac{\partial \boldsymbol{u}_{\varepsilon}}{\partial t} \cdot \frac{\partial \boldsymbol{\phi}_{\varepsilon}}{\partial t}=\int_{\Omega \times(0, T) \times Y} \rho \frac{\partial \boldsymbol{u}_{0}}{\partial t} \cdot \frac{\partial \boldsymbol{w}_{0}}{\partial t}$ $d x d t d y=\int_{0}^{T}\left(\xi^{\prime}, \tilde{\xi}^{\prime}\right)_{H} d t$, hence

$$
\lim _{\varepsilon \rightarrow 0} J_{2 \varepsilon}=\tilde{a}(\xi, \tilde{\xi}) .
$$


The convergences deduced by substituting 1 for $\rho_{\varepsilon}$ and $\rho$ in (7.10) hold true, hence

$$
\begin{aligned}
\lim _{\varepsilon \rightarrow 0} \int_{\Omega \times(0, T)} \rho_{\varepsilon}\left|\frac{\partial \phi_{\varepsilon}}{\partial t}\right|^{2} d x d t & =\int_{\Omega \times(0, T) \times Y} \rho\left|\frac{\partial \boldsymbol{w}_{0}}{\partial t}\right|^{2} d x d t d y \\
& =\int_{0}^{T}\left(\tilde{\xi}^{\prime}, \tilde{\xi}^{\prime}\right)_{H} d t
\end{aligned}
$$

We deduce from an explicit computation that

$$
\begin{aligned}
\varepsilon \boldsymbol{e}\left(\boldsymbol{\phi}_{\varepsilon}\right) 1_{\Omega \backslash B_{\varepsilon}} \longrightarrow \boldsymbol{e}_{y}\left(\boldsymbol{w}_{0}\right) 1_{Y \backslash B}, & \\
\boldsymbol{e}\left(\boldsymbol{\phi}_{\varepsilon}\right) 1_{B_{\varepsilon}} \longrightarrow & \longrightarrow\left(\begin{array}{ccc}
\frac{-l}{2(l+1)} \frac{\partial \psi_{3}}{\partial x_{3}} & 0 & -\frac{1}{2} \frac{\partial \varphi}{\partial x_{3}}\left(\boldsymbol{y}-\boldsymbol{y}_{B}\right)_{2} \\
0 & \frac{-l}{2(l+1)} \frac{\partial \psi_{3}}{\partial x_{3}} & \frac{1}{2} \frac{\partial \varphi}{\partial x_{3}}\left(\boldsymbol{y}-\boldsymbol{y}_{B}\right)_{1} \\
-\frac{1}{2} \frac{\partial \varphi}{\partial x_{3}}\left(\boldsymbol{y}-\boldsymbol{y}_{B}\right)_{2} & \frac{1}{2} \frac{\partial \varphi}{\partial x_{3}}\left(\boldsymbol{y}-\boldsymbol{y}_{B}\right)_{1} & \frac{\partial \psi_{3}}{\partial x_{3}}
\end{array}\right) 1_{B}(y),
\end{aligned}
$$

yielding, in accordance with (7.13), (7.15), (7.19), (7.22), (7.52)

$$
\lim _{\varepsilon \rightarrow 0} J_{3 \varepsilon}=\tilde{a}(\tilde{\xi}, \tilde{\xi}) .
$$

Joining (7.48), (7.50), (7.51), (7.53), and taking the strong two-scale convergence of $\left(\boldsymbol{u}_{0}\left(x, t, \frac{x}{\varepsilon}\right)-\boldsymbol{\phi}_{\varepsilon}\right)$ to $\boldsymbol{u}_{0}-\boldsymbol{w}_{0}$ into account (cf. (2.23)), we infer

$$
\begin{aligned}
\limsup _{\varepsilon \rightarrow 0} & \left\|\boldsymbol{u}_{0}\left(x, t, \frac{x}{\varepsilon}\right)-\boldsymbol{u}_{\varepsilon}\right\|_{L^{2}}^{2} \\
& \leq C \limsup _{\varepsilon \rightarrow 0} \int_{\Omega \times(0, T)}\left|\boldsymbol{u}_{0}\left(x, t, \frac{x}{\varepsilon}\right)-\boldsymbol{\phi}_{\varepsilon}\right|^{2}+\left|\boldsymbol{u}_{\varepsilon}-\boldsymbol{\phi}_{\varepsilon}\right|^{2} d x d t \\
& \leq C \int_{0}^{T}|\xi-\tilde{\xi}|_{H}^{2} d t+C \tilde{a}(\xi-\tilde{\xi}, \xi-\tilde{\xi})+C|(\xi-\tilde{\xi})(0)|_{H}^{2} .
\end{aligned}
$$

By the arbitrary choice of $\tilde{\xi} \in C^{\infty}([0, T] ; W)\left(W:=\left\{\left(\boldsymbol{w}_{0}, \boldsymbol{\psi}, \varphi\right) \in V, \boldsymbol{w}_{0} \in \mathcal{D}(\Omega\right.\right.$; $\left.\left.\left.C_{\sharp}^{\infty}\left(Y ; \mathbb{R}^{3}\right)\right)\right\}\right)$, the density of $C^{\infty}([0, T] ; W)$ in $W^{1,2}(0, T ; V, H)$ and the continuity of the application $\zeta \rightarrow \int_{0}^{T}|\zeta|_{H}^{2} d t+\tilde{a}(\zeta, \zeta)+\int_{\Omega \times Y}|\zeta(0)|^{2} d x d y$ on $W^{1,2}(0, T ; V, H)$, the corrector result (2.25) is proved. The convergence $\boldsymbol{u}_{\varepsilon} \longrightarrow \boldsymbol{u}_{0}$ follows then from (2.23). Remark 7.1. If $\boldsymbol{u}_{\varepsilon} \longrightarrow \boldsymbol{u}_{0}$, then by Fatou's Lemma

$$
\int_{\Omega \times(0, T) \times Y}\left|\boldsymbol{u}_{0}\right|^{2} d x d \tau d y=\lim _{\varepsilon \rightarrow 0} \int_{0}^{T} \int_{\Omega}\left|\boldsymbol{u}_{\varepsilon}(\tau)\right|^{2} d x d \tau \geq \int_{0}^{T}\left(\liminf _{\varepsilon \rightarrow 0} \int_{\Omega}\left|\boldsymbol{u}_{\varepsilon}(\tau)\right|^{2} d x\right) d t .
$$

On the other hand, as for all $\tau$ there holds $\boldsymbol{u}_{\varepsilon}(\tau) \longrightarrow \boldsymbol{u}_{0}(\tau)$, we have (see [2], Theorem 0.2)

$$
\liminf _{\varepsilon \rightarrow 0} \int_{\Omega}\left|\boldsymbol{u}_{\varepsilon}(\tau)\right|^{2} d x \geq \int_{\Omega \times Y}\left|\boldsymbol{u}_{0}(\tau)\right|^{2} d x d y, \forall \tau \in[0, T]
$$

thus $\liminf _{\varepsilon \rightarrow 0} \int_{\Omega}\left|\boldsymbol{u}_{\varepsilon}(\tau)\right|^{2} d x=\int_{\Omega \times Y}\left|\boldsymbol{u}_{0}(\tau)\right|^{2} d x d y$, for a.e. $\tau \in[0, T]$. Hence for a.e. $\tau \in[0, T]$, the sequence $\left(\boldsymbol{u}_{\varepsilon}(\tau)\right)$ two-scale converges strongly, up to a subsequence, to $\boldsymbol{u}_{0}(\tau)$. 
8. Proof of Theorem 3.1. The first step consists in the study of the asymptotic behavior of some sequences associated with the sequence $\left(\boldsymbol{u}_{\varepsilon}\right)$ of the solutions of (1.1). Repeating the argument of the proof of Proposition 6.4, we obtain $\int_{\Omega}\left(\rho_{\varepsilon}\left|\frac{\partial \boldsymbol{u}_{\varepsilon}}{\partial t}\right|^{2}+\varepsilon^{2}\left|\boldsymbol{e}\left(\boldsymbol{u}_{\varepsilon}\right)\right|^{2}+\mu_{1 \varepsilon}\left|\boldsymbol{e}\left(\boldsymbol{u}_{\varepsilon}\right)\right|^{2} 1_{B_{\varepsilon}}\right)(\tau) d x \leq C, \quad \forall \tau \in[0, T]$, and applying (6.14) to $\boldsymbol{w}=\boldsymbol{u}_{\varepsilon}$, get $\int_{\Omega}\left|\boldsymbol{u}_{\varepsilon}\right|^{2}(\tau) d x \leq C$ and then $\int_{\Omega}\left(\left|\boldsymbol{v}_{\varepsilon}\right|^{2}+\left|\boldsymbol{r}_{\varepsilon}\right|^{2}\right)(\tau) d x \leq C$ (see $(2.16),(3.3))$. We infer that, up to a subsequence, there holds

$$
\begin{aligned}
& \boldsymbol{u}_{\varepsilon} \longrightarrow \boldsymbol{u}_{0}, \quad \frac{\partial \boldsymbol{u}_{\varepsilon}}{\partial t} \longrightarrow \frac{\partial \boldsymbol{u}_{0}}{\partial t}, \quad \varepsilon \boldsymbol{e}\left(\boldsymbol{u}_{\varepsilon}\right) 1_{\Omega} \longrightarrow \boldsymbol{\Xi}^{m}, \quad \varepsilon \boldsymbol{e}\left(\boldsymbol{u}_{\varepsilon}\right) 1_{B_{\varepsilon}} \longrightarrow 0, \\
& \boldsymbol{u}_{\varepsilon} \stackrel{\star}{*} \boldsymbol{u}, \quad \boldsymbol{v}_{\varepsilon} \stackrel{\star}{*} \boldsymbol{v}, \quad \boldsymbol{r}_{\varepsilon} \stackrel{\star}{\rightarrow} \boldsymbol{r} \quad \text { star-weakly in } L^{\infty}\left(0, T ; L^{2}\right) .
\end{aligned}
$$

We identify $\boldsymbol{\Xi}^{m}=\boldsymbol{e}_{y}\left(\boldsymbol{u}_{0}\right)$, deduce that $\boldsymbol{u}_{0} \in L^{\infty}\left(0, T ; L^{2}\left(\Omega ; H_{\sharp}^{1}\left(Y ; \mathbb{R}^{3}\right)\right)\right)$ and that $\boldsymbol{e}_{y}\left(\boldsymbol{u}_{0}\right)=0$ in $\Omega \times(0, T) \times B$, hence $\boldsymbol{u}_{0}=\boldsymbol{a}+\boldsymbol{b} \wedge\left(\boldsymbol{y}-\boldsymbol{y}_{B}\right)$ for a suitable $(\boldsymbol{a}, \boldsymbol{b}) \in$ $\left(L^{\infty}\left(0, T ; L^{2}\left(\Omega ; \mathbb{R}^{3}\right)\right)\right)^{2}$. We find $\boldsymbol{a}=\boldsymbol{v}$ (see (6.36)), then fixing $\boldsymbol{\gamma} \in \mathcal{D}\left(\Omega \times(0, T) ; \mathbb{R}^{3}\right)$, deduce from (3.3), (8.1) that

$$
\begin{aligned}
\int_{\Omega \times(0, T)} \boldsymbol{r} \cdot \boldsymbol{\gamma} d x d t & =\lim _{\varepsilon \rightarrow 0} \int_{B_{\varepsilon} \times(0, T)} \boldsymbol{J}^{-1}\left(\left(\left[\frac{\boldsymbol{x}}{\varepsilon}\right]-\boldsymbol{y}_{B}\right) \wedge \boldsymbol{u}_{\varepsilon}\right) \cdot \gamma d x d t \\
& =\int_{\Omega \times(0, T) \times B} \boldsymbol{J}^{-1}\left(\left(\boldsymbol{y}-\boldsymbol{y}_{B}\right) \wedge \boldsymbol{u}_{0}\right) \cdot \gamma d x d t d y \\
& =\int_{\Omega \times(0, T)} \boldsymbol{J}^{-1}\left(\int_{B}\left(\boldsymbol{y}-\boldsymbol{y}_{B}\right) \wedge\left(\boldsymbol{b} \wedge\left(\boldsymbol{y}-\boldsymbol{y}_{B}\right)\right) d y\right) \cdot \gamma d x d t \\
& =\int_{\Omega \times(0, T)}\left(\boldsymbol{J}^{-1} \boldsymbol{J} \boldsymbol{b}\right) \cdot \boldsymbol{\gamma} d x d t=\int_{\Omega \times(0, T)} \boldsymbol{b} \cdot \boldsymbol{\gamma} d x d t
\end{aligned}
$$

thus $\boldsymbol{b}=\boldsymbol{r}$. The next step consists in the choice of a suitable sequence of test fields. We define

$$
\begin{aligned}
& H^{(4)}:=\left\{\left(\boldsymbol{w}_{0}, \boldsymbol{\psi}, \boldsymbol{\gamma}\right) \in L^{2}\left(\Omega \times Y ; \mathbb{R}^{3}\right) \times\left(L^{2}\left(\Omega ; \mathbb{R}^{3}\right)\right)^{2},\right. \\
& \left.\quad \boldsymbol{w}_{0}(x, y)=\boldsymbol{\psi}+\gamma \wedge\left(\boldsymbol{y}-\boldsymbol{y}_{B}\right) \text { in } \Omega \times B\right\}, \\
& \left(\left(\boldsymbol{w}_{0}, \boldsymbol{\psi}, \boldsymbol{\gamma}\right),\left(\tilde{\boldsymbol{w}}_{0}, \tilde{\boldsymbol{\psi}}, \tilde{\boldsymbol{\gamma}}\right)\right)_{H^{(4)}}:=\int_{\Omega \times Y} \rho \boldsymbol{w}_{0} \cdot \tilde{\boldsymbol{w}}_{0} d x d y,
\end{aligned}
$$

choose $\left(\boldsymbol{w}_{0}, \boldsymbol{\psi}, \boldsymbol{\gamma}\right) \in L^{2}\left(0, T ; H^{(4)}\right)$ satisfying (7.2), (7.3), and set

$$
\boldsymbol{\chi}_{\varepsilon}(x, t, y):=\widehat{\boldsymbol{\psi}}_{\varepsilon}(x, t)+\widehat{\boldsymbol{\gamma}}_{\varepsilon}(x, t) \wedge\left(\boldsymbol{y}-\boldsymbol{y}_{B}\right)-\boldsymbol{w}_{0}(x, t, y),
$$

where $\widehat{\boldsymbol{\psi}}_{\varepsilon}$ and $\widehat{\boldsymbol{\gamma}}_{\varepsilon}$ are given by (6.15). We multiply (1.1) by $\phi_{\varepsilon}:=\eta_{\varepsilon}\left(\frac{x}{\varepsilon}\right) \boldsymbol{\chi}_{\varepsilon}\left(x, t, \frac{x}{\varepsilon}\right)+$ $\boldsymbol{w}_{0}\left(x, t, \frac{x}{\varepsilon}\right)$, where $\eta_{\varepsilon}$ is defined by (7.5) and get (7.8). We obtain (7.11), set (7.12), find (7.14), (7.16) and $I_{3 \varepsilon}=0$ and, passing to the limit as $\varepsilon \rightarrow 0$ in (7.8), get

$$
\begin{aligned}
& \int_{\Omega \times(0, T) \times Y} \rho \boldsymbol{u}_{0} \cdot \frac{\partial^{2} \boldsymbol{w}_{0}}{\partial t^{2}} d x d t d y+\int_{\Omega \times Y} \rho \boldsymbol{a}_{0} \cdot \frac{\partial \boldsymbol{w}_{0}}{\partial t}(0) d x d y-\int_{\Omega \times Y} \rho \boldsymbol{b}_{0} \cdot \boldsymbol{w}_{0}(0) d x d y \\
& \quad+\int_{\Omega \times(0, T) \times(Y \backslash B)} \boldsymbol{e}_{y}\left(\boldsymbol{u}_{0}\right): \boldsymbol{\sigma}_{0 y}\left(\boldsymbol{w}_{0}\right) d x d t d y=\int_{\Omega \times(0, T) \times Y} \rho \boldsymbol{f} . \boldsymbol{w}_{0} d x d t d y .
\end{aligned}
$$


We set

$$
\begin{aligned}
& V^{(4)}:=\left\{\tilde{\xi}=\left(\boldsymbol{w}_{0}, \boldsymbol{\psi}, \boldsymbol{\gamma}\right) \in H^{(4)}, \boldsymbol{w}_{0} \in L^{2}\left(\Omega ; H_{\sharp}^{1}\left(Y ; \mathbb{R}^{3}\right)\right)\right\}, \\
& \xi:=\left(\boldsymbol{u}_{0}, \boldsymbol{v}, \boldsymbol{r}\right), \xi_{0}^{(4)}:=\left(\boldsymbol{a}_{0}, \boldsymbol{a}_{0}, 0\right), \xi_{1}^{(4)}:=\left(\boldsymbol{b}_{0}, \boldsymbol{b}_{0}, 0\right), h^{(4)}:=(\boldsymbol{f}, \boldsymbol{f}, 0), \\
& ((\xi, \tilde{\xi}))_{V^{(4)}}:=(\xi, \tilde{\xi})_{H^{(4)}}+\int_{\Omega \times Y \backslash B} \boldsymbol{\nabla}_{y} \boldsymbol{u}_{0} \cdot \boldsymbol{\nabla}_{y} \boldsymbol{w}_{0} d x d y, \\
& a^{(4)}\left(\left(\boldsymbol{u}_{0}, \boldsymbol{v}, \boldsymbol{r}\right),\left(\boldsymbol{w}_{0}, \boldsymbol{\psi}, \boldsymbol{\gamma}\right)\right):=\int_{\Omega \times(Y \backslash B)} \boldsymbol{e}_{y}\left(\boldsymbol{u}_{0}\right): \boldsymbol{\sigma}_{y}\left(\boldsymbol{w}_{0}\right) d x d y, \quad \bar{a}^{(4)}:=0 .
\end{aligned}
$$

Since there holds $\xi \in L^{2}\left(0, T ; V^{(4)}\right), \xi^{\prime} \in L^{2}\left(0, T ; H^{(4)}\right)$, the variational formulation (8.5) is equivalent to (6.10). By Korn's inequality we have $\|\tilde{\xi}\|_{V^{(4)}}^{2} \leq C\left(|\tilde{\xi}|_{H^{(4)}}^{2}+\right.$ $a^{(4)}(\tilde{\xi}, \tilde{\xi})$ ), yielding $(6.9)$. We deduce from Theorem 6.2 that $\left(\boldsymbol{u}_{0}, \boldsymbol{v}, \boldsymbol{r}\right)$ is the unique solution of (6.10) and satisfies the properties of continuity and the initial boundary conditions stated in (3.5). By integrating (8.5) with respect to $y$ over $B$, thanks to (2.12), (3.3), we obtain

$$
\begin{gathered}
\int_{\Omega \times(0, T) \times(Y \backslash B)} \rho \boldsymbol{u}_{0} \cdot \frac{\partial^{2} \boldsymbol{w}_{0}}{\partial t^{2}} d x d t d y+\int_{\Omega \times(Y \backslash B)} \rho \boldsymbol{a}_{0} \cdot \frac{\partial \boldsymbol{w}_{0}}{\partial t}(0) d x d y \\
-\int_{\Omega \times(Y \backslash B)} \rho \boldsymbol{b}_{0} \cdot \boldsymbol{w}_{0}(0) d x d y+\int_{\Omega \times(0, T) \times(Y \backslash B)} \boldsymbol{e}_{y}\left(\boldsymbol{u}_{0}\right): \boldsymbol{\sigma}_{0 y}\left(\boldsymbol{w}_{0}\right) d x d t d y \\
+\int_{\Omega \times(0, T)}\left(\bar{\rho}_{1} \boldsymbol{v}+\bar{\rho}_{1} \boldsymbol{r} \wedge\left(\boldsymbol{y}_{G}-\boldsymbol{y}_{B}\right)\right) \cdot \frac{\partial^{2} \boldsymbol{\psi}}{\partial t^{2}} d x d t+\int_{\Omega} \bar{\rho}_{1} \boldsymbol{a}_{0} \cdot \frac{\partial \boldsymbol{\psi}}{\partial t}(0) d x \\
-\int_{\Omega} \bar{\rho}_{1} \boldsymbol{b}_{0} \cdot \boldsymbol{\psi}(0) d x+\int_{\Omega \times(0, T)}\left(\boldsymbol{J}^{\rho} \cdot \boldsymbol{r}+\bar{\rho}_{1}\left(\left(\boldsymbol{y}_{G}-\boldsymbol{y}_{B}\right) \wedge \boldsymbol{v}\right)\right) \cdot \frac{\partial^{2} \boldsymbol{\gamma}}{\partial t^{2}} d x d t \\
+\int_{\Omega} \bar{\rho}_{1}\left(\left(\boldsymbol{y}_{G}-\boldsymbol{y}_{B}\right) \wedge \boldsymbol{a}_{0}\right) \cdot \frac{\partial \boldsymbol{\gamma}}{\partial t}(0) d x-\int_{\Omega} \bar{\rho}_{1}\left(\left(\boldsymbol{y}_{G}-\boldsymbol{y}_{B}\right) \wedge \boldsymbol{b}_{0}\right) \cdot \boldsymbol{\gamma}(0) d x \\
=\int_{\Omega \times(0, T) \times(Y \backslash B)} \rho \boldsymbol{f} \cdot \boldsymbol{w}_{0} d x d t d y+\int_{\Omega \times(0, T)} \bar{\rho}_{1} \boldsymbol{f} \cdot \boldsymbol{\psi} d x d t \\
+\int_{\Omega \times(0, T)} \bar{\rho}_{1}\left(\left(\boldsymbol{y}_{G}-\boldsymbol{y}_{B}\right) \wedge \boldsymbol{f}\right) \cdot \boldsymbol{\gamma} d x d t .
\end{gathered}
$$

Choosing $\boldsymbol{\psi}=\gamma=0$ in (8.7) we deduce (7.30), (7.31) and find the equation obtained by replacing $\boldsymbol{m}\left(\boldsymbol{u}_{0}\right) \cdot \boldsymbol{e}_{3} \varphi$ by $\boldsymbol{m}\left(\boldsymbol{u}_{0}\right) \cdot \boldsymbol{\gamma}$ in (7.33). Subtracting it from (8.7), we get

$$
\begin{aligned}
& \int_{\Omega \times(0, T)}\left(\bar{\rho}_{1} \boldsymbol{v}+\bar{\rho}_{1} \boldsymbol{r} \wedge\left(\boldsymbol{y}_{G}-\boldsymbol{y}_{B}\right)\right) \cdot \frac{\partial^{2} \boldsymbol{\psi}}{\partial t^{2}} d x d t+\int_{\Omega} \bar{\rho}_{1} \boldsymbol{a}_{0} \cdot \frac{\partial \boldsymbol{\psi}}{\partial t}(0) d x \\
& -\int_{\Omega} \bar{\rho}_{1} \boldsymbol{b}_{0} \cdot \boldsymbol{\psi}(0) d x+\int_{\Omega \times(0, T)}\left(\boldsymbol{J}^{\rho} \boldsymbol{r}+\bar{\rho}_{1}\left(\left(\boldsymbol{y}_{G}-\boldsymbol{y}_{B}\right) \wedge \boldsymbol{v}\right)\right) \frac{\partial^{2} \boldsymbol{\gamma}}{\partial t^{2}} d x d t \\
& +\int_{\Omega} \bar{\rho}_{1}\left(\left(\boldsymbol{y}_{G}-\boldsymbol{y}_{B}\right) \wedge \boldsymbol{a}_{0}\right) \cdot \frac{\partial \boldsymbol{\gamma}}{\partial t}(0) d x-\int_{\Omega} \bar{\rho}_{1}\left(\left(\boldsymbol{y}_{G}-\boldsymbol{y}_{B}\right) \wedge \boldsymbol{b}_{0}\right) \cdot \boldsymbol{\gamma}(0) d x \\
& =\int_{\Omega \times(0, T)}\left(\bar{\rho}_{1} \boldsymbol{f}+\boldsymbol{g}\left(\boldsymbol{u}_{0}\right)\right) \cdot \boldsymbol{\psi} d x d t+\int_{\Omega \times(0, T)}\left(\bar{\rho}_{1}\left(\left(\boldsymbol{y}_{G}-\boldsymbol{y}_{B}\right) \wedge \boldsymbol{f}\right)+\boldsymbol{m}\left(\boldsymbol{u}_{0}\right)\right) \cdot \boldsymbol{\gamma} d x d t
\end{aligned}
$$

yielding the equations satisfied by $(\boldsymbol{v}, \boldsymbol{r})$ set forth in (3.5). The corrector result is obtained by fitting the argument of the fibered case.

Remark 8.1. In the fibered case, by substituting $\theta \boldsymbol{e}_{3}$ for $\boldsymbol{b}$ in (8.2), we find that the sequence $\left(\boldsymbol{r}_{\varepsilon}\right)$ converges star-weakly in $L^{\infty}\left(0, T ; L^{2}\left(\Omega: \mathbb{R}^{3}\right)\right.$ to $\boldsymbol{r}:=\theta \boldsymbol{e}_{3}$. 
9. Sketch of the proof of Proposition 5.2. a) $(v) \Rightarrow$ (iii). If (a) (resp. (b)) is satisfied, the proof of the estimate (5.3) is similar to that of the estimate below formula (4.32) of [9] (resp. Formula (4.3) of [9]).

$($ iii $) \Rightarrow($ iv $)$. By multiplying (5.1) by $\boldsymbol{u}_{\varepsilon}$ and by integrating by parts, we infer from (5.3) that $\left(\boldsymbol{u}_{\varepsilon}\right)$ is bounded in $L^{2}\left(\Omega ; \mathbb{R}^{3}\right)$.

$(i v) \Rightarrow(v)$. Assume by contradiction that neither (a) nor (b) are satisfied, then the dimension of the subspace of $\mathbb{R}^{3}$ spanned by the directions of the fibers is lower than or equal to 2 . We can assume without loss of generality that this subspace is spanned by $\left(\boldsymbol{e}_{2}, \boldsymbol{e}_{3}\right)$. Fix $\boldsymbol{f}:=\boldsymbol{e}_{1}$. By $(i v),\left(\boldsymbol{u}_{\varepsilon}\right)$ admits a two-scale converging subsequence which by Corollary 5.1 satisfies (5.2). Consider the constant field $\boldsymbol{w}_{0}(x, y):=\boldsymbol{e}_{1}$. It can be checked that $\boldsymbol{w}_{0} \in V, a\left(\boldsymbol{u}_{0}, \boldsymbol{w}_{0}\right)=0,\left(\boldsymbol{f}, \boldsymbol{w}_{0}\right)_{H} \neq 0$, hence (5.2) has no solution, a contradiction.

$($ iii $) \Rightarrow(i)$. We choose a smooth field $\boldsymbol{w}_{0} \in V$ and consider the sequence of test field $\left(\phi_{\varepsilon}\right)$ corresponding to that introduced in the proof of Theorem 2.1. Repeating the argument of the proof of (7.53), we get $\lim _{\varepsilon \rightarrow 0} F_{\varepsilon}\left(\phi_{\varepsilon}\right)=a\left(\boldsymbol{w}_{0}, \boldsymbol{w}_{0}\right)$. Passing to the limit as $\varepsilon \rightarrow 0$ in the inequality $\left\|\phi_{\varepsilon}\right\|_{L^{2}\left(\Omega ; \mathbb{R}^{3}\right)}^{2} \leq C F_{\varepsilon}\left(\phi_{\varepsilon}\right)$, we infer $\left\|\boldsymbol{w}_{0}\right\|_{L^{2}\left(\Omega \times Y ; \mathbb{R}^{3}\right)}^{2} \leq$ $C a\left(\boldsymbol{w}_{0}, \boldsymbol{w}_{0}\right)$. Thanks to (4.2), (4.3) and to Korn's inequality in $H^{1}\left(Y \backslash B ; \mathbb{R}^{3}\right)$, we get $\left|\boldsymbol{w}_{0}\right|_{V}^{2} \leq C a\left(\boldsymbol{w}_{0}, \boldsymbol{w}_{0}\right)$.

$(i) \Rightarrow(i i)$. This results from the Lax-Milgram Theorem.

$(i i) \Rightarrow(v)$. Similar to the proof of $(i v) \Rightarrow(v)$.

$(v i) \Rightarrow(i v)$. Obvious.

$($ iii $) \Rightarrow(v i)$. If (iii) holds, then $\left(\boldsymbol{u}_{\varepsilon}\right)$ is bounded in $L^{2}\left(\Omega ; \mathbb{R}^{3}\right)$ (see the proof of $($ iii $) \Rightarrow(i v)$ ) and that (5.2) has a unique solution $\boldsymbol{u}_{0}$ (because $($ iii $) \Rightarrow($ ii)). Hence, by Corollary 5.1, $\left(\boldsymbol{u}_{\varepsilon}\right)$ two-scale converges to $\boldsymbol{u}_{0}$.

b) Assume by contradiction that (5.2) has a solution $\boldsymbol{u}_{0}$. Let $P$ denote the subspace of $\mathbb{R}^{3}$ orthogonal to the space spanned by the directions of the fibers. Fix $\boldsymbol{w} \in \mathcal{D}(\Omega)$ such that $\boldsymbol{w}(x) \in P, \forall x \in \Omega$ and $(\boldsymbol{f}, \boldsymbol{w})_{L^{2}\left(\Omega ; \mathbb{R}^{3}\right)}>0$. Set $\boldsymbol{w}_{0}(x, y):=\boldsymbol{w}(x)$. Then $\boldsymbol{w}_{0} \in V$ and $\left(f, \boldsymbol{w}_{0}\right)_{H}>0$. On the other hand, since (v) b) is not satisfied and since $\boldsymbol{w}_{0}(x, y) \in P$, we infer $a\left(\boldsymbol{u}_{0}, \boldsymbol{w}_{0}\right)=0$, which contradicts (5.2).

\section{REFERENCES}

[1] Z. Abdessamad, I. Kostin, G. Panasenko, V. P. Smyshlyaev, Homogenization of thermoviscoelastic Kelvin Voigt model, Comptes Rendus Mécanique,vol. 335, Issue 8 (2007), pp. $423-429$.

[2] G. Allaire, Homogenization and two-scale convergence, SIAM J. Math. Anal., 23 (1992), pp. $1482-1518$.

[3] Y. Amirat, K. Hamdache, A. Ziani, Etude d'une équation de transport à mémoire, C. R. Acad. Sci. Paris Sér. I Math., 311 (1990), pp. 685-688.

[4] T. Arbogast, J. Douglas, U. Hornung, Derivation of the double porosity model of single phase flow via homogenization theory, SIAM J. Math. Anal., 21 (1990), pp. 823-836.

[5] N.O. Babych, I.V. Kamotski, V.P. Smyshlyaev, Homogenization in periodic media with doubly high contrasts, Networks and heterogeneous Media, 3 (3) (2008), 413-436.

[6] M. Bellieud, Homogenization of evolution problems in a fiber reinforced structure, J. Convex Anal., 11, No. 2 (2004), pp. 363-385.

[7] - Homogenization of evolution problems for a composite medium with very small and heavy inclusions, ESAIM Control Opt. Calc. Var., 11 (2005), pp. 266-284.

[8] - Vibrations d'un composite élastique comportant des inclusions granulaires lourdes, Comptes rendus Mathématique, Vol. 346, Issue 13-14, pp. 807-812 (2008).

[9] M. Bellieud, G. Bouchitté, Homogenization of a soft elastic material reinforced by fibers, Asymptot. Anal., 32, No. 2 ( 2002), pp. 153-183.

[10] M. Bellieud, I. Gruais, Homogenization of an elastic material reinforced by very stiff or 
heavy fibers. Non local effects. Memory effects, J. Math. Pures Appl., 84 (2005), pp. $55-96$.

[11] A. Braides, M. BRiane, Homogenization of non-linear variational problems with thin lowconducting layers, Appl. Math. Optim., 55, No. 1 (2007), pp. 1-29.

[12] M. Camar-Eddine, P. Seppecher, Determination of the closure of the set of elasticity functionals, Arch. Ration. Mech. Anal., 170 (2003), pp. 211-245.

[13] K. B. Cherednichenko, Two-scale asymptotics for non-local effects in composites with highly anisotropic fibres, Asymptotic analysis, 49 (2006), pp. 39-59.

[14] K. B. Cherednichenko, V. P. Smyshlyaev, V. V. Zhikov, Non-local homogenised limits for composite media with highly anisotropic periodic fibres, Proceedings of the Royal Society of Edinburgh: Section A 136 (2006), pp. 87-114.

[15] G. Dal Maso, An introduction to $\Gamma$-Convergence, Progress in non linear diff. eq. and their app., Birkhauser, Boston (1993).

[16] R. Dautray, J.L. Lions, Analyse mathématique et calcul numérique pour les sciences et les techniques, 8, Evolution: semi-groupe, variationnel, Masson (1988).

[17] E. Y. Khruslov, Homogenized models of composite media, Progress in Nonlinear Differential Equations and their Application, Birkhäuser (1991), pp. 159-182.

[18] J. L. Lions, Equations différentielles opérationnelles et problèmes aux limites, Grundlehren Bd. 111, Berlin / Göttingen / Heidelberg: Springer (1961).

[19] J. L. Lions, E. Magenes, Problèmes aux limites non homogènes et applications, 1, Dunod, Paris (1968).

[20] A. E. H. Love, A treatrise on the mathematical theory of elasticity, New York Dover Publications (1944).

[21] M. L. Mascarenhas, Memory effects phenomena and Gamma-convergence, Proc. Roy. Soc. Edinburgh, 123 A (1993), pp. 311-322.

[22] U. Mosco, Composite media and asymptotic Dirichlet forms, J. Funct. Anal., 123 (1994), pp. $368-421$.

[23] G. NGuetseng, A general convergence result for a functional related to the theory of homogenization, SIAM J. Math. Anal., 20 (1989), pp. 608-623.

[24] O. A. Oleinik, A. S. Shamaev, G. A. Yosifian, Mathematical problems in elasticity and homogenization, Stud. Math. Appl., North Holland (1992).

[25] G. P. PANASEnKo, Multicomponent homogenization of processes in strongly nonhomogeneous structures, Sb. Math. vol. 69, no. 1 (1991), pp. 143-153.

[26] - Multicomponent homogenization of the vibration problem for incompressible media with heavy and rigid inclusions, C.R. Acad. Sci. Paris, t. 321, Série I, (1995), pp. 1109-1114.

[27] C. Pideri, P. Seppecher, A second gradient material resulting from the homogenization of an heterogeneous linear elastic medium, Contin. Mech. Thermodyn., 9 (1997), pp. 241-257.

[28] G.V. SANDRAKOV, Homogenization of elasticity equations with contrasting coefficients (Russian), Mat. Sbornik, 190 (12) (1999), p.p. 37-92; English translation in Sbornik Math., 190 (12) (1999), p.p. 1749-1806., Zh. Vychisl. Mat. i Mat. Fiz., 44:10 (2004), p.p. 1829-1844; English transl. Comput. Math. Math. Phys., 44:10 (2004), pp. 1741-1756.

[29] - Multiphase models of nonstationary diffusion in homogenization, Zh. Vychisl. Mat. i Mat. Fiz., 44:10 (2004), p.p. 1829-1844; English transl. Comput. Math. Math. Phys., 44:10 (2004), pp. 1741-1756.

[30] — Multiphase homogenized diffusion models for problems with several parameters, Izvestiya RAN: Ser. Mat. 71:6 (2007), pp. 119-182, English transl. Izvestiya: Mathematics $71: 6$ (2007), pp. 1193-1252.

[31] A. Sili, Homogenization of the linearized system of elasticity in anisotropic heterogeneous thin cylinders, Math. Meth. Appl. Sci. vol. 25, Issue 4 (2002), pp. 263-288.

[32] L. Tartar, Memory effects and Homogenization, Arch. Rat. Mech. Anal. vol. III, no 2, pp. 121-133, 1990.

[33] — Cours Peccot, Collège de France, (1977), unpublished, partially written in F. Murat, L. TARTAR, "H-convergence", in Topics in the Mathematical Modelling of Composite Materials, ed. by L. Cherkatev, R. V. Kohn, Progress in Nonlinear Differential Equations and their Applications, 31, Birkaüser, Boston (1998), pp. 21-43. 